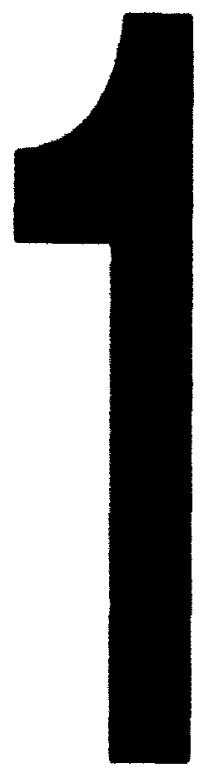

Pu-1 3\%"x4" Photoenapuc unchocopy taRest

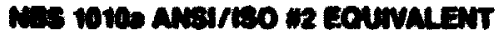

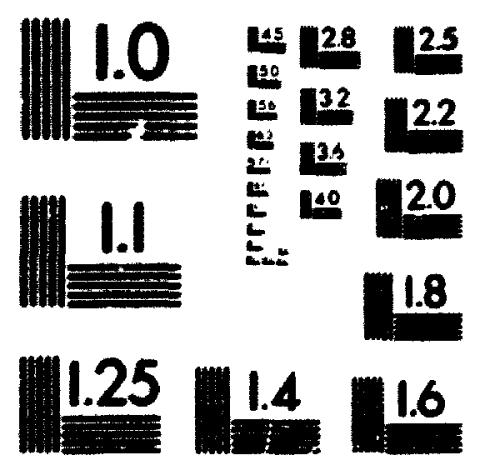

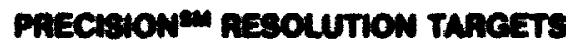


National Library

of Canada

Acquisitions and

Bibliographic Services Branch

395 Wellington Street

Onawa. Ontario

K1A ON4

\section{Bibliotheque nationale}

du Canada

Direction des acquisitions et des services bibliographiques

395. rue Wellington

Ottawa (Ontaro)

KIA ONA
The quality of this microform is heavily dependent upon the quality of the original thesis submitted for microfilming. Every effort has been made to ensure the highest quality of reproduction possible.

If pages are missing, contact the university which granted the degree.

Some pages may have indistinct print especially if the original pages were typed with a poor typewriter ribbon or if the university sent us an inferior photocopy.
La qualité de cette microforme dépend grandement de la qualité de la these soumise au microfilmage. Nous avons tout fait pour assurer une qualité supérieure de reproduction.

S'il manque des pages, veuillez communiquer avec l'université qui a conféré le grade.

La qualité d'impression de certaines pages peut laisser à désirer, surtout si les pages originales ont été dactylographiées à l'aide d'un ruban usé ou si l'universitó nous a fait parvenir une photocopie de qualité inférieure.

La reproduction, même partielle, de cette microforme est soumise à la Loi canadienne sur le droit d'auteur, SRC 1970, c. C-30, et ses amendements subséquents.
Reproduction in full or in part of this microform is governed by the Canadian Copyright Act, R.S.C. 1970, c. C-30, and subsequent amendments. 


\title{
Radial Basis Function Equalizer for Mobile Channels
}

\author{
By \\ P. Roohi Larijani , B.Sc.
}

\author{
A thesis Submitted to \\ the Faculty of Graduate Studies and Research \\ in partial fulfillment of \\ the requirements for the degree of \\ Master of Engineering.
}

Department of Systems and Computer Engineering

Carleton University

Ottawa, Ontario, Canada K1S $5 B 6$

March 1994

(C) copyright

P.R.Larijani 
National Library

of Canada

Acquisitions and Bibliographic Services Branch

305 Wellingtion Sireet Ottawa, Onlario

KIA ONIA
Biblichtoque nationale

du Canada

Direction des acquisitions et

des senvices bibliographiques

395. ne Wellington

Orima (Ontario)

KIAONA

Your be votre itterence

Ou ace nowe reterence

The author has granted an irrevocable non-exclusive licence allowing the National Lbrary of Canada to reproduce, loan, distribute or sell coples of his/her thesis by any means and in any form or format, making this thesis available to interested persons.
L'auteur a accordé une licence irrévocable et non exclusive permettant à la Bibliothéque nationale du Canada de reproduire, prêter, distribuer ou vendre des coples de sa thèse de quelque manière et sous quelque forme que ce solt pour mettre des exemplaires de cette thèse a la disposition des personnes intéressées.

L'auteur conserve la propriété du droit d'auteur qui protège $8 a$ these. Ni la thèse ni des extraits substantiels de celle-ci ne doivent être imprimés ou autrement reproduits sans son autorisation.

ISBN $\quad 0-315-92948-0$ 
Name Parsya Ranh Larizami

Dissertation Abstrdets Internotional is orranged by brood, general subject cotegories. Flease select the one wbject which most neorty describes the content of your dissentotion. Enter the corresponding four-digit code in the spoces provided.

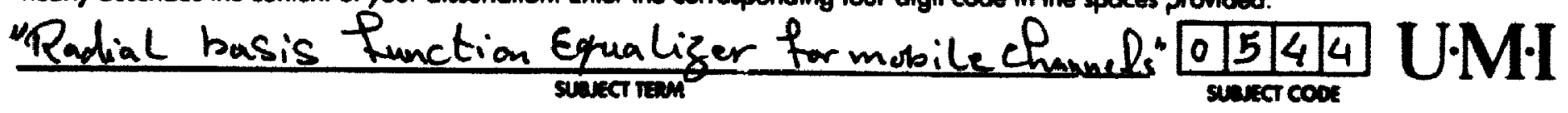

\section{Subinat Congorim}

\section{Tw womanums and socul senness}

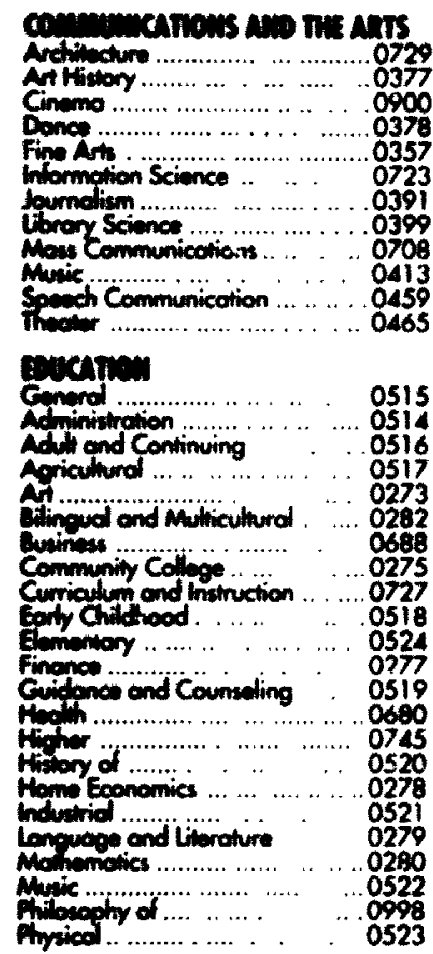

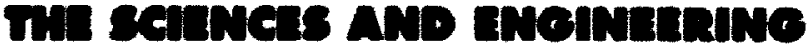

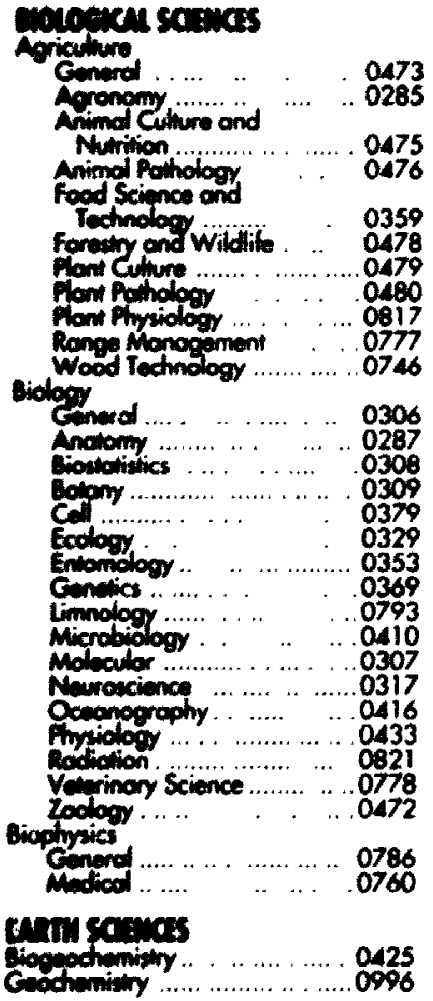

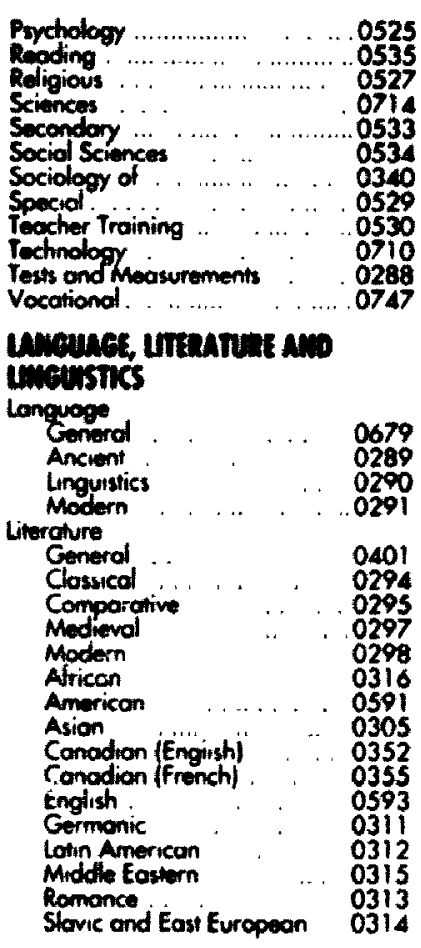

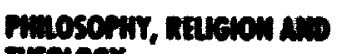

\section{Thiolor}

Philonophy

Roligim

Ganerol $\ldots . . . . . . . .0318$

Biblicol Studies . .......0321

Clergy of $\quad \ldots \ldots . \quad \ldots .0319$

Philosopty of ................0322

Theology .............................0469

come satuces

Ampricon Studies ..............0323

Antropology

Anchomology ...... ..... 0324

Culural

Plunice ...................0326

Business Administrotion

Generol

Accounting.....

Boniting

Manogement

Morkeling

Conodion

General

Agricultural.

Commerce-Business

Finance

Histion

Theor

folltiore

Geogrophy

Gorontology

Generd
Anciont ......................0579

Modievol .......................... 0589

Modorn .........................0582

Aricon ….....................

Asia, Austrotia ond Oceonia 0332

Conodion ......................... 033

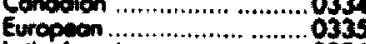

Lurtin Avnericon .........................0336

Midole Eoskern …...........0333

Unind Shows .....................0337

low . O scince.....................03\%

Political Science

Generd.

Internotionol low and

.0615

Relation

0816

Public Administration ............ . 817

Rocreation .............................0814

Soxiaboy

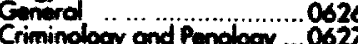

Criminology and Penology ....0627

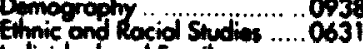

individual ond fomity

Shudiet ........................0826

Industrial ond lebor

Relation ....................0629

Public ond Sociol Walfore ....0630

Sociol Structure ond

Demopment ............. .. 0700

iheory ond Methook ...........034

Ironsportution ........

Women's Studies ...................0453

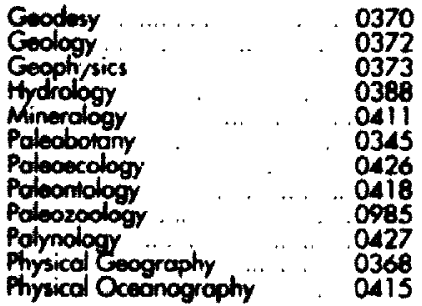

"rum an exmoumenta

\section{scancs}

Environmental Sciences

0768

Hooth Sciences

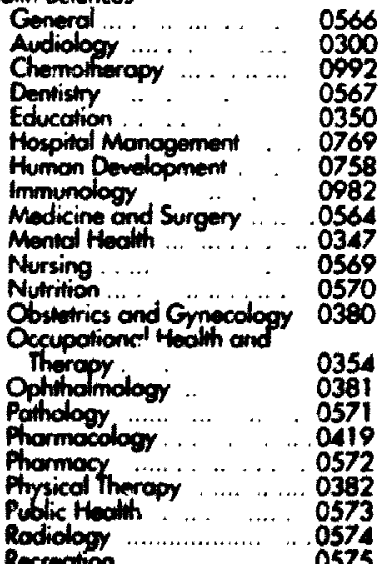

Recreotion \begin{tabular}{lll}
0370 & Speoch Pothology &. \\
0372 & .0460 \\
\hline & Toxicology & 0383
\end{tabular} Home Economics $\quad 0386$

\section{mricen satiogs}

Pure Sciences

Chemistiry

Agricuturo

Anricuturcal

Biochemistry

Inorganie

Orgonic

Pharmoceutical

Physical

Radiotion

Mothemotics

Physics

Generol

Acoustics

Astronomy and

Astrophysics. .... 0606

Almospherir Scrence ... 0608

Alamic .. .........0748

Electronics and Electricin 0607

Elementory Ponticles ond

High Energr

Fund and Plormo

Molecular ............... 0609

Nucleor . .....0610

Optics

Solidition.

0752

Stotistics

0611

Apind Simase

Applied Machonics

Computar Science
Enginearing

$\begin{array}{lll}\text { Aerospace } & \ldots & \ldots . .0537\end{array}$

Agriculurol. . ............. 0539

Ainomative ............... 05.

Biomedied .....................054

Chemical ..................... 054

Eivil ................ Es and

thot and Thermodmamics.. 034

Hydroulie .......... ..........0545

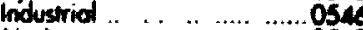

Morine .. .. ........... 050

Moteriols Science ........... 079

Mochonicet ............... 0548

Metallurey .. ... .................0743

Mining

Nucleor

Podaging ... . . ............... OS

05s1

Sortos

Sonitary and Municipal .... ...055

Systom Seience ... ... . ......... .0790

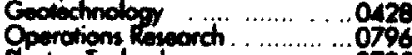

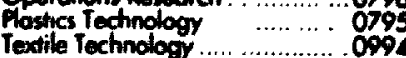

crounary

Generd .. … . . .. ..........0621

ethovigral ... .. .......... 038

Clinicol ...... .....................0622

Experimental . ..................

holvstrial ......................62

Porsondity . ..................625

mysiological. .... .............0989

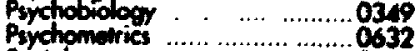

Socid .................................0451 
The undersigned recommend to the Faculty of Graduate Studies and Research acceptance of the thesis

"Radial Basis Function Equalizer for Mobile Channels"

Submitted by P.Roohi Larijani, B. SC.

in partial fulfillment of the requirements for

the degree of Master of Engineering
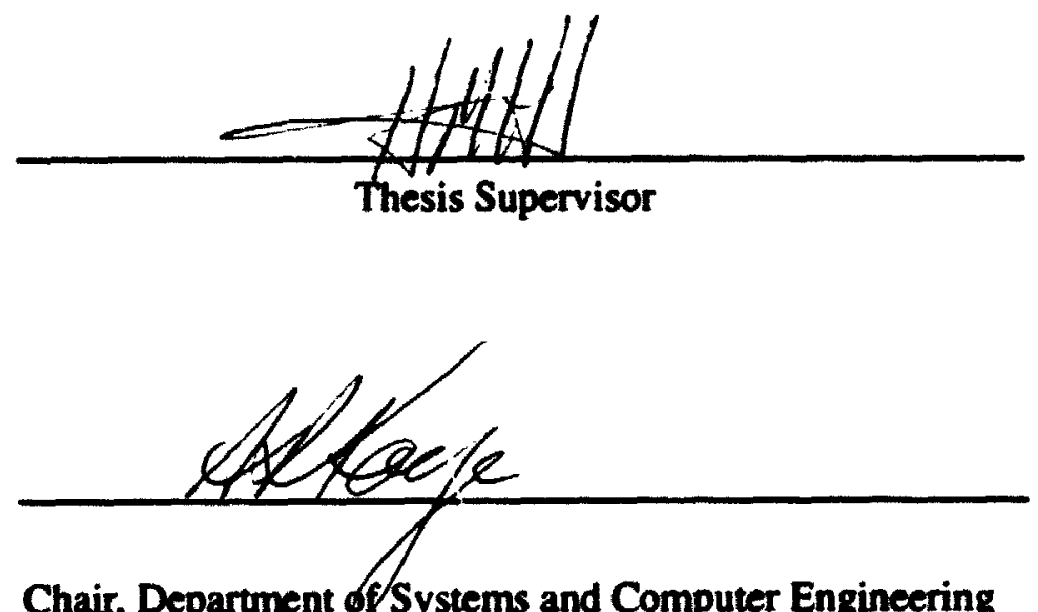

Chair, Department of Systems and Computer Engineering

Carleton University

March 14, 1994 


\section{Abstract}

Excessive delay spread in mobile channels causes severe inter symbol-interference. The problem can be corrected by adaptive equalizers that implicitly estimares the channel impulse response and filter the received signal through the inverse channel. For reliable communications, the adaptive filter should be able to converge quickly, and maintain its convergence in the presence of occasional errors and fading hits.

Least mean square (LMS) adaptation algorithm is commonly used to adapt equalizer 'weights because of its simplicity. The LMS algorithm, in its stochustic gradient form. adapts the equalizers weights using a fixed small step size and hence it is too slow to cope with rapid variations in the channel impulse response.

In this thesis we introduce a new adaptation algorithm for the decision feedback equalizers. The adaptation of the equalizer taps is based on a new cost function which adjusts the step size automatically to speed up the convergence. The choice of the cost function in this algorithm is derived from a simple geometrical interpretation of the equalization process under multipath conditions.

To evaluate the equalizer performance, analytical method and direct computer simuiation are used. In analytical approach mathematical expressions for convergence speed and steady state error level are derived. Analytical results are confirmed by simulation. 


\section{Acknowledgments}

I wish to express my sincere gratitude to my thesis supervisor Prof. H. M. Hafez for his consistence guidance and encouragement during the course of this thesis research.

I would also like to thank Mr. A. Chini for some simulating discussions on aspects of our theses. Furthermore, I am appreciative of the pleasant friendships which I have made with fellow graduate students.

Most importantly, I am indebted to my family for their gentle encouragement. In particular, I thank my parents for their unconditional love and sense of perspective. 


\section{TABLE OF CONTENTS}

Abotract

Acknowledgments

II

List of Abbreviations and Acronyms

III

List of Matbenatical Symbols and Notations

VI

LIST OF FIGURES

VII

Compler1

Introduction

1.1 INTRODUCTION:

1

1.2 THESIS DESCRIPTION:

1.3 THESIS ORGANIZATION:

Chapter 2

Back Ground and Related research

2.1 INTER-SYMBOL-NTERFERENCE (ISI):

4

2.2 ADAPTIVE EQUALIZATION TECHNIQUES:

2.3 THE RADIAL BASIS FUNCTION EQUALIZER: 8

Chapter 3

Syctem Description

14

3.1 SYSTEM MODEL: $\quad 14$

3.2 MODULATION:

3.3 DATA STRUCTURE:

3.4 TRANSMIT AND RECEIVE FILTERS: 16

3.5 THE NOISE CHARACTERISTICS: 16

3.6 THE CHANNEL MODELS: $\quad 20$

3.7 RECEIVER STRUCTURE:

3.8 DESCRIPTION OF THE SIMULATION PROGRAM: 29

Chapler 4

Simolation Resulls and Performance Analyais 30

4.1 NTRODUCTION: $\quad 30$

4.2 R.B.F ALGORTHM WITH SINGLE GAUSSIAN TERM: $\quad 30$

4.2.1 STABIITY CONDITION: 32

4.2.2 CONVERGENCE RATE: 34

4.2.3 STEADY STATE BEHAVIOR ANALYSIS: 
4.2.4 SIMULATION RESULTS:

4.2.5 COMPUTATIONAL COMPLEXITY: 39

4.3 BLOCK RADIAL BASIS FUNCTION EQUALIZER: 54

4.3.1 INTRODUCTION: $\quad 54$

4.3.2 BLOCK R.B.F ALGORITHM:

4.3.3 ANALYSIS: $\quad 56$

4.3.4 SMMUATION RESULTS:

4.3.5 COMPUTATIONAL COMPLEXITY: $\quad 62$

4.4 MODIFIED B.R.B.F ALGORITHM:

Chapter 5

Conclusions and recommendations for future research 82

REFERENCES: $\quad 84$

APPENDXX A: $\quad 88$

APPENDXX B:

APPENDXX C: 101 


\section{List of Abbreviations and Acronyms}

ACI

AWGN

B.R.B.F

$\mathrm{CCI}$

CIR

dB

DFE

LE

$\mathrm{Hz}$

ISI

LMS

MMSE

MSE

MLSE

QPSK

RLS

R.B.F

$\mathbf{S} / \mathbf{N}$
Adjacent channel interference

Additive white Gaussian noise

Block Radial Basis Function

Cochannel interference

Channel Impulse Response

Decibel

Decision feedback equalizer

Linear equalizer

Hertz

Intersymbol interference

Least mean square error

Minimum mean square error

Mean square error

Maximum likelihood sequence estimator

Quadrature phase shift keying

Recursive least square

Radial basis function

Signal to noise ratio 


\section{List of Mathematical Symbols and Notations}

\begin{tabular}{|c|c|}
\hline$E_{b}$ & Energy per bit \\
\hline $\mathbf{E}[]$. & Expectation of the argument \\
\hline ert & Error value \\
\hline$E r_{e x}$ & Excess mean square error \\
\hline $\mathbf{F}_{\mathbf{d}}$ & Maximum Doppler frequency \\
\hline$h_{\text {src }}(t)$ & Impulse response of the square root raised cosine filter \\
\hline I & Identity matrix \\
\hline $\mathbf{M}$ & Misadjustment factor \\
\hline MSE & Mean square error \\
\hline N1 & Number of forward taps \\
\hline N2 & Number of feedback taps \\
\hline$n(t)$ & Filtered complex valued AWGN \\
\hline $\mathbf{N}_{\mathbf{0}}$ & Two sided power spectral density of complex AWGN \\
\hline $\mathbf{P}$ & Cross correlation matrix \\
\hline $\mathbf{R}$ & Autocorrelation matrix \\
\hline $\mathbf{T}$ & Symbol period \\
\hline $\mathbf{T}(\mathbf{x})$ & Equalizer cost function \\
\hline $\mathbf{W}$ & Vector of equalizer tap weight coefficients \\
\hline$w_{e}$ & Error weight vector \\
\hline
\end{tabular}


$\mathbf{W}_{\text {opt }}$

$\mathbf{X}$

$x^{*}$

Yd

$\mathbf{Y}$

$\mathbf{Z}$

B

$\boldsymbol{\beta}_{\max }$

$\beta_{\min }$

$\beta_{\text {eq }}$

$\lambda_{i}$

$\tau$

$\sigma_{\mathrm{a}}$

$\boldsymbol{\sigma}$
Optimum equalizer weight vector

Input vector

Complex conjugate of Input vector

Desired data

Equalizer output

Complex gaussian process

Equalizer adaptation rate

Maximum adaptation rate

Minimum adaptation rate

Equivalent adaptation rate

$i^{\text {th }}$ eigenvalue of auto correlation matrix

time constant of convergence speed

Variance of the data

Radial basis function cluster width 


\section{LIST OF FIGURES}

Figure (3.1) System model..........................................................................15

Figure (3.2) Square root raised cosine filter...........................................18

Figure (3.3) Signalling format ...................................................................19

Figure (3.4) T-spaced tapped delay line model for a 2-ray multipath fading channel....................................................................................................22

Figure (3.5) 2 equal power ray fading channel ...........................................23

Figure (3.6) T/2-spaced tapped Delay Line model for a 3-ray multipath fading channel.................................................................................24

Figure (4.1) Set up for channel estimation.................................................40

Figure(4.2) Channel estimator at zero Doppler freq. and noise variance:.01

Figure (4.3) Channel esaimator at zero Doppler freq. and noise variance:.03 ...............................................................................................42

Figure (4.4) Channel estimator at Doppler freq: $2 \mathrm{~Hz}$..................................43

Figure (4.5 ) Channel estimator at Doppler freq: $10 \mathrm{~Hz}$...........................44

Figure (4.6) Channel estimator at Doppler freq:20 Hz ..............................45

Figure (4.7) comparing LMS and R.B.F channel estimator ....................46

Figure (4.8) Equalizer performance at zero Dopfler freq. and S/

$\mathrm{N}=20 \mathrm{~dB}$

Figure (4.9) Equalizer pertiormance at zero Doppler freq. and S/

$\mathrm{N}=15 \mathrm{~dB}$ .48

Figure (4.10) Equalizer performance at doppler freq. $=2 \mathrm{~Hz}$....................49

Figure (4.11) Equalizer performance at doppler freq. $=10 \mathrm{~Hz}$

Figure (4.i2) Equalizer performance at doppler freq. $=15 \mathrm{~Hz} \ldots \ldots \ldots \ldots \ldots \ldots . . .51$

Figure (4.13) Training overhead ......................................................52

Figure (4.14) Comparing LMS and RBF Equalizer.................................53 
Figure (4.15): Geometrical interpretation of R.A.P algorithm ...................58

Figure (4.16) Convergence speed / variance parameter..............................63

Figure (4.17) Convergence rate/Block length.........................................64

Figure (4.18) Symbol rate/Block length ..............................................65

Figure (4.19) Equalizers's Converọence rate............................................66

Figure (4.20) Comparing different equalizer performances ....................67

Figure (4.21) Symbol error rate /Doppler frequency at three ray

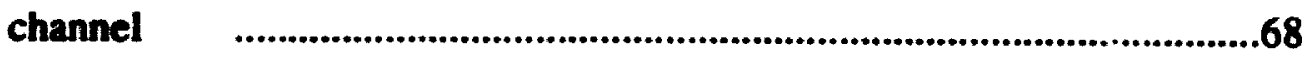

Figure (4.22) Symbol error rate /Doppler frequency at two ray channel

Figure (4.23) Two branch diversity with two separate adaptive equalizers. .70

Figure (4.24) Performance of equalizer with diversity................................71

Figure (4.25) Error propagation effect.........................................................72

Figure(4.26) Noise efiect with considering error propagation ..................73

Figure (4.27) Computational complexity..................................................74

Figure (4.28) modified cost function ........................................................76

Figure (4.29) Fading channel sample function .......................................79

Figure (4.30) Equalizer output MSE.......................................................80

Figure (4.31) Modified B.R.B.F algorithm performance............................81 


\section{Chapter1 Introduction}

\subsection{INTRODUCTION:}

The mobile radio channel is characterized by multipath propagation and frequent signal fading. The complexity of the mobile channel model depends on many factors, including the propagation terrain, the speed of the receiver and the relation between the signal bandwidth and the channel coherence bandwidth.

The limited available radio spectrum and the phenomenal increase in demands on mobile applications are forcing system designers to increase the transmitted bits/sec/Hz/unit area. This packing of the spectrum is challenging the mobile receivers with high levels of intersymbol-interferences (ISI), co-channel interference (CCI), adjacent channel interference (ACI) $[1,23,28]$, time-varying channel impulse response, random phase distortion due to Doppler effect and frequency selective fading. Channel equalization using fast adaptive filters is an effective technique to deal with some of these impairments.

The newly proposed Northern American digital cellular standard (IS-54) $[13,18,41]$ is based on a narrow-band time division multiplex access (TDMA) strategy. While the channel bandwidth in this standard is relatively small (30 kHz), the data rate is high (24 $\mathrm{ks} / \mathrm{s}$ or equivalently $48 \mathrm{kbps}$ ). The channel specifications state that the delay spread in some area could be as high as one full symbol period. In order to meet this criterion under fast fading conditions, the channel equalizer must possess superior convergence and tracking characteristics. Traditional equalization techniques; such as: linear equalizers (LE), decision feed-back equalizers (DFE) and maximum likelihood sequence estimation (MLSE) must be re-designed for higher cr. nvergence speed under time varying conditions. 
The main problem facing the designers of the IS-54 equipment is that the fading index, which is defined in [29] as the product of the Doppler frequency and the symbol duration is ten times larger in the IS-54 than in the European GSM system [12]. This means that, in the IS-54 standard, the channel is assumed to be changing quite fast, which necessitates the development of equalization techniques with fast convergence and good tracking capabilities.

\subsection{THESIS DESCRIPTION:}

In a slow varying channel, the equalization problem is quite tractable. A typical solution would involve a DFE of sufficient length. If the length of the DFE spans most of the channel impulse response, the equalizer can reduce the ISI (and the interference) to an acceptable level. However, time variation in mobile channel can be quite severe during long data blocks. The DFE is sensitive to channel variations, and in order to keep it on track, we either have to increase the frequency of training (at the expense of reducing the system throughput) or modify the equalizer's weight updating algorithm to improve its tracking capability.

The sensitivity of the LMS DFE is not due to its structure, but rather it is due to the LMS adaptation algorithm. The most common method of applying the LMS algorithm is the stochastic gradient descent, where the weight taps are adjusted by a small fixed step size after each decision. The choice of the step size is a trade-off between small residual error (small steps), and fast converyence (large steps). The step size is limited to the range $0<\beta<\left(2 / \lambda_{\max }\right)$ [39] where $\lambda_{\max }$ is the largest eigenvalue of the received signal covariance matrix. In a time varying channel, the selection of the step size faces two problems: first, $\lambda_{\max }$ is variable so that the choice of a large step size could lead to divergence, and second, when the signal fades or varies significantly, the small (ard safe) step size hinders the equalizer's ability to rz-converge quickly.

An alternative approach to the stochastic LMS algorithm is the recursive least squares (RLS) technique [39]. The RLS adapts the equalizer by estimating the covariance matrix of several exponentially weighted channel observations. The estimated covariance emphasizes the recent observation by weighting them more heavily in the calculations. It is known that the RLS tracks the channel variations much closer than LMS. It is also known that the RLS involves extensive computations and is vulnerable to numerical instability [38][15]. 
In this thesis, a new gradient-based algorithm is proposed to improve the performance of LMS equalizers in time-varying channels. The new technique is based on what is known in the neural networks feld as the radial basis function network (RBF). It is slightly more complex than standard LMS equalizer but it improves the speed of convergence and reduce the sensitivity to channel variations.

Channel impairments considered in this work are intersymbol-interference (ISI) and additive white Gaussian noise and Doppler fading. We do not investigate the effects of adjacent channel interftrence $(\mathrm{ACl})$ and co-channel interference $(\mathrm{CCl})$.

\subsection{THESIS ORGAVIZATION:}

This thesis is organized as follow:

In Chapter 2, background relevant to the problems investigated in the thesis is presented. In particular, we discuss different equalization techniques and compare them in terms of performance and complexity. The radial basis function network is introduced and its applicability to the problem of channel equalization is discussed.

In chapter 3, the communication system model which has been simulated and used during this work is explained.

Chapter 4 contains the simulation results and related analysis.

Finally, in chapter 5. we complete the thesis with a concluding discussion. 


\section{Chapter 2 \\ Back Ground and Related research}

\subsection{INTER-SYMBOL-INTERFERENCE (ISI):}

In most digital transmission systems, the symbol waveforms are not time-limited. The system is normally designed such that the combined effects of the transmitter and receiver filter produce zero inter-symbol-interference (ISI) at the correct sampling instants. However, when the signal is transmitted over channels with unknown or variable delay or when the channel exhibits multipath or other forms of delay distortion the signal will suffer from ISI [38] .

The ISI effect can be illustrated by the following equation:

$$
r(t)=\sum_{k} a(k T) g(t-k T)+n(t)
$$

where $r(t)$ is received signal, $g(t)$ is the overall channel impulse response, $a(k T)$ is the transmitted symbol at time $t=k T$ and $n(t)$ is additive white Gaussian noise.

The ISI impairment can be reduced by using appropriately band limited signals which do not cause ISI. Such an ISI-free condition requires the overall channel impulse response be zero at all sampling instants [38] except at the desired sampling instant $(t=0)$. Based on Nyquist criterion, the folded spectrum of the sampled overall channel impulse response must have a constant gain in Nyquist bandwidth as given below.

$$
\frac{1}{T} \sum_{k} G\left(f-\frac{k}{T}\right)=1 \quad U \leq \frac{1}{2 T}
$$

Low pass filters with excess bandwidth and odd symmetry about $\frac{1}{2 T} \mathrm{~Hz}$ satisfy the Nyquist criterion. For example, a filter with raised-cosine frequency response [39] satisfies the Eq(2.2) for any excess bandwidth parameters between 0 and unity.

The use of excess bandwidth filtering doesn't produce any ISI but causes aliasing distortion at all times except the sampling instants. This aliasing; however, could be acceptable since informaticn signalling occurs only at the data sampling instants and not in the actual waveform. Aliasing distortion will cause problem if the receiver sampling is not precisely synchronized with the received signals. 
In mobile channels, adaptive equalization is required even if we use Nyquist filtering, since multipath fading channel can still cause time dispersion in the transmitted signal.

\subsection{ADAPTIVE EQUALIZATION TECHNIQUES:}

Equalization techniques for combating intersymbol interference on band limited time dispersive channels may be subdivided into two general types: Linear and non-linear equalization. Each type has one or more structures for implementing the equalizer. Furthermore, for each structure there are class of algorithms that may be employed to adapt the equalizet parameters according to some specified performance criterion [37].

The linear equalizer (LE) has the simplest structure. It essentially attempts to invert the folded spectrum of the overall channel impulse response such that the cascade of the channel and the equalizer satisfies the Nyquist criterion. A major disadvantage of the linear equalizer is the fact that it suffers from unacceptable noise enhancement when there is severe amplitude distortion [38,39], and as such it is not a good choice for fading channels where deep fades often occur.

Nonlinear equalizers are less susceptible to the noise enhancement problem, since they don't attempt to invert the folded spectrum of the channel. Two important nonlinear equalizers are: (1) the maximum likelihood sequence estumator (MLSE) and (2) Decision fesdback equalizer (DFE).

Maximum likelihood equalizer yields optimum performance close to that of the matched fitter bound. However, this performance is achieved at a cost in processing delay and complexity. Indeed, MLSE computational complexity increases exponentially with the multipath spread and can be prohibitively high for very dispersive channels [38].

The second group of nonlinear equalizer (DFE) has a performance slightly inferior to MLSE, provided that there is no error propagation. Furthermore, the DFE has the advantage of being of similar complexity as the linear equalizer but much less complex than the MLSE. The decision feed back equalizer is comprised of a forward filter and a feedback filter.

The forward filter removes the precursor ISI, while the backward filter removes the post cursor ISI by subtracting the filtered detected symbols from the output of the forward filter. Assuming that most decisions are correct, the removal of the post cursor ISI doesn't induce any noise enhancement, and since the forward filter only removes the 
precursor ISI it is subject to less noise enhancement than if it had to remove all the ISI. The disadvantage of using DFE stems frum it's sensitivity to error propagation, where decision error generate an incorrect post cursor ISI estimate which will further increase the probability of decision errors. This is the main problem related to employing DFE in fast fading channels where deep fades can severely degrade their performance.

"The criterion most commonly used in the optimization of the equalizer coefficients is the minimization of the mean square error (MSE) between the desired equalizer output and the actual equalizer output. The minimization of the MSE results (in the well known optimum) Wiener filter solution for the coefficients vector, which may be expressed as

$$
w_{o p t}=\Gamma^{-1} \xi
$$

where $\Gamma$ is the autocorrelation matrix of the vector of signal samples in the equalizer at any given time instant and $\xi$ is the vector of cross correlations between the desired data symbols and the signal samples in the equalizer"[37].

An alternative approsch for minimization of the MSE is stochastic gradient algorithm introduced by B. Widrow [44]. This algorithm is described by the following coefficient update equation

$$
w_{k+1}=w_{k}+\beta e_{k} x_{k}^{*}
$$

where $\boldsymbol{W}_{k}$ is the vector of the equalizer coefficients at the $k^{\text {th }}$ iteration,$X_{k}^{*}$ is the complex conjugate of the signal samples vector at the kth iteration, $q_{z}$ is the error signal, which is defined as the difference between the transmitted symbol $I_{k}$ and its corresponding estimate at the output of the equalizer, $\beta$ is the step size parameter that controls the rate of adjustment.

"It is well known that the step size parameter $\beta$ controls the rate of adaptation of the equalizer'[37] and that the stability of the LMS algorithm requires that $\beta$ be limited to the range of $0<\beta<\lambda_{\max }$ where $\lambda_{\max }$ is the largest eigenvalue of the signal covariance matrix. Choosing a large value for $\beta$ just below the upper limit provides apid convergence, but it also introduces large fuctuations in the equalizer coefficients during steady-state operation. Consequently, "the choice of $\beta$ involves a trade-off between rapid convergence and the desire to keep the variance of the steady state crror small'[37]. 
An alternative for LMS adaptation is employing a recursive least squares (RLS) criterion for adjustment of the equalizer coefficients, which is based on minimizing a sum of exponentially weighted squared errors i.e

$$
\begin{aligned}
& E=\sum_{n=0}^{k} w^{k-n} \times\left|I_{n}-I_{n}\right|^{2} \\
& =\sum_{n=0}^{k} w^{k-n} \times\left|I_{n}-w_{k} x^{*}\right|^{2}
\end{aligned}
$$

by minimizing the above expression for error the following equation for updating the equalizer weights is obtained:

$$
w_{k+1}=w_{k}+P_{k} x_{k}^{*} e_{k}
$$

where $\mathfrak{L}_{k}$ is the estimate of the kth symbol $\left(\boldsymbol{L}_{k}\right)$ at the output of the equalizer, $W_{k}^{\prime}$ is the transpose of $W_{k}$ (the equalizer's coefficient vector) and ek $=l_{k}-I_{k}$

$$
P_{k}=\frac{1}{w}\left[P_{k-1}-\frac{P_{k-1} X_{k}^{*} X_{k}^{\prime} P_{k-1}}{w+X_{k-1} P_{k} X_{k}^{\prime}}\right]
$$

the weighting factor $w$ varies in the range $0<w<1, P_{k}$ is an $(N \times N)$ square matrix which is the inverse of the auto-correlation matrix:

$$
R_{k}=\sum_{n=0}^{k} w^{k-n} X^{k} X_{n}
$$

The following table compare the computational complexity of LMS and RLS algorithm for a decision feedbeck equalizer's structure: 
TABLE 1.computational complexity of adaptive DFE algorithm [38]

$\begin{array}{ll}\text { Algorithm } & \text { Total number of complex operations } \\ \text { LMS DFE } & 2 \mathrm{~N}+1 \\ \text { Fast RLS (Kalman) DFE } & 20 \mathrm{~N}+5 \\ \text { Conventional RLS DFE } & 2.5 \mathrm{~N}^{\wedge} 2+4.5 \mathrm{~N} \\ \text { Square-root RLS DFE } & 1.5 \mathrm{~N} 2+6.5 \mathrm{~N} \\ \text { Gradient lattice DFE } & 13 \mathrm{~N} 1+33 \mathrm{~N} 2-36 \\ \text { RLS lattice DFE } & 18 \mathrm{~N} 1+39 \mathrm{~N} 2-39\end{array}$

$N$ is total equalizer length [feed forward and feed beck filters]

N1 is number of coefficients in the feed forward filter

N2 is the number of coefficients in the feed beck fitter

A third algorithm for adaptation is probabilistic algorithms. The maximum a-posterior probability (M.A.P) algorithm and the maximum likelihood sequence estimator (MLSE) are optimal in the sense that they minimize the probability of the error. In the M.A.P algorithm, it is the symbol error rate that is minimized and in MLSE the Viterbi algorithm minimizes the probability of a sequence of errors .

Bawed on this brief review of different types of equalizers, it is obvious that to equalize a time-varying channel, one needs an adaptation algorithm with performance comparable to the RLS and a computational complexity comparable to the LMS algorithm.

\section{THE RADIAL BASIS FUNCTION EQUALIZER:}

In this section we introduce the concept of the Radial basis function network and explain it's applicability to the channel equalization problem.

The RBF is a concept used frequently in the area of neural networts, and has been applied to several signal processing and clustering problems in the past few years 
$[35,4,41,11]$. In applying the $R B F$, one assumes that an observed random vector, $X$, is a member of some known (or assumed) multidimensional probability density function (pdf). The pdf is the RBF, $\phi(X)$. The shape of the RBF is known, but its center is unknown. The objective of the adaptation process is to locate the center of the RBF using a series of observed random vectors $\left\{X_{i}\right\}$.

The RBF can be used to adapt an equalizer tap vector, W, by assuming that the dot product of $\mathbf{W}$ and the signal vector $X$ is a member of a certain pdf. The center of the pdf is the correct symbol, $d$. Therefore, the cost function associated with an RBF equalizer can be expressed as:

$$
f_{i}=\lambda_{0}+\lambda \cdot\left(\mid W \times X-d_{d}\right)
$$

$\lambda_{0}$ and $\lambda$ are two constants, and $\phi($.$) is a nonlinear function with radially symmetric$ shape. Although there is a variety of choices for this nonlinear function two typical choices are

$$
\begin{gathered}
\phi(r, \sigma)=\exp \left( \pm r^{2} / \sigma^{2}\right) \\
\phi(r ; 1)=r^{2} \log (r)
\end{gathered}
$$

Since the channel vectors are shifted from their nominal positions by Gaussian noise and several multipath mys, the Gaussian form of Eq (2.10) is more appropriate in our application.

Using an RBF to process complex signals can be viewed as performing a curve-fitting operation in a multi-dimensional space in order to realize some complex non-linear decision function or to approximate certain complicated data generation mechanism.

If the poff of the received signal vectors has a complex shape, which cannot be represented by a single RBF, several RBF could be used, one for each part of the complex paf. In mobile communications, the appropriate choice of the paf is a mixed Gauscian distribution.

It is known that an equalizer (or more gencrally a neural networt) with large enough number of RBF's can spproximnte the pdf of the unequalized received signal or any continuous function to within an atbitrary accuracy $[34,36]$. 
The RBF method has been successfully used for channel equalization by other rusearchers $[5,6,17]$. Their work was based on the observation that the channel equalization can be viewed as an optimum decision boundary approximation in the space of the received signal vectors, and that the equalizer is to approximate this decision boundary. They have employed a Gaussian shape for the radial functions, and realized the equalizer as a neural network. Testing the proposed RBF equalizer for a time invariant channel resulted in a performance that is significantly better than LMS DFE at the expense of more handware complexity.

We offer two specific critiques to the work reported in $[5,6,17]$. First, there is no need for many RBF. The extra performance enhancement gained by trying to closely approximate the pdf produces diminishing gain as the number of RBF increases. Second, the use of the multi-layer neural structure creale a complex error function with local minime. Hinton [20-21] considered these problems, and proposed a much simpler RBF structure for blind channel equalization. The equalizer proposed by Hinton has a conventional structure (FIR filter), but it uses a radial basis function as a cost function to optimize the filier ups. The equalizer assumes that the output (rather than the received vector) belongs to one of $L$ clusters, one cluster for each possible symbol. There is one radial basis function for each cluster. The shape of all the RBF's is the same, they only differ in their center locations.

Accordingly, Hinton formulated the following cost function:

$$
O(i)=\sum_{i} a_{i} \cdot \exp \left(-\left|Y-d_{d}\right|^{2} / \sigma^{2}\right)
$$

$d_{i}$ is desired output signal sonstellation point, $\sigma$ is noise variance, $a_{i}$ is a weighting coefficient and $Y$ is the equalizer output This cost function was maximized over an FIR filter by gradient deacent algorithm. In this work, the channel was time invariant and the only impairment was additive white Gaussian noise.

Karagoz [25] tried the same cost function for a blind equalizer in time variable channel and they concluded that this equalizer can coaverge very fast in a closed eye sibution. The signal constellation in [25] had two or four points.

Chen [7] modified this algorithm for high dimensional signal constellations and applied it for blind channel equalization. 
In this thesis, we investigate performance of decision feed back equalizer with Gaussian cost function in a supervised mode rather than blind equalization. Three different types of radial basis cost functions have been investigated: first, a single exponential term cost function is studied which is the building block of the other more complex structure. In this case

$$
\exp \left(\left|\left(y_{o}-X d\right)\right|^{2} /\left(2 \sigma^{2}\right)\right)
$$

is the function which has to be minimized. In this expression, $y_{0}$ is the output of equalizer, $X d$ is the desired data and $\sigma$ is radial basis function width. Ideally $\sigma$ should be set equals to the noise standard deviation.

The second cost function which is called block radial basis is:

$$
\sum_{i=1}^{L} \exp \left(\left|\left(y o_{i}-x d_{i}\right)\right|^{2} /\left(2 \sigma_{l}^{2}\right)\right)
$$

where $y \mathrm{y}_{i}$ is equalizer output at time instant $i, x d_{i}$ is desired output at instant $i$ and $L$ is data block length.

It should be noted that the Block RBF adaptation rule is based on gradient descent algorithm and from this point of view it is similar to the LMS and it uses blocks of recent data to compute the error term, and from this viewpoint it is similar to the RLS.

Third cost function considered is (modified Block R.B.F) :

$$
\sum_{j=1}^{L} T_{j}
$$

where $T_{\mathbf{j}}$ is :

$$
T_{j}=\sum_{i=1}^{n} \exp \left(\left|\left(y o_{j}-x d_{i}\right)\right|^{2} /\left(2 \sigma_{i}^{2}\right)\right)
$$

yo is equalizer output, $x d$ is a signal constellation point, $\sigma_{i}$ is the ith cluster width. and $n$ is the number of points in signal constellation. For exampie, in the case of QPSK ( $n=4)$ ,$L$ is the block length. 
Other notable attempts to modify the basic LMS algorithm in order to make it suitable for time varying channels include the followings:

1-Eleftheriou Falconer [14] proposed a novel modification of the simple LMS algorithm which improves it's tracking ability. This involved preceding the LMS DFE with an adaptive lattice whitening filter to reduce eigenvalues spread of the received signal. This rechnique improved convergence speed and tracking behavior of LMS equalizer considerably at the expense of an increase in complexity.

2-Vandamme \& Leclert [27] modified the coefficient updating algorithm of the LMSDFE as follow:

$$
\begin{gathered}
P_{k}^{n+1}=P_{k}^{n}-\mu \times e_{n} \times S_{n-k} \\
q_{m}^{n+1}=q_{m}^{n}-\mu \times\left[e_{n} \times a_{n-m}\right]+v \times\left\{\operatorname{sgn}\left(R E\left[q_{m}\right]\right)+\operatorname{sgn}\left(\operatorname{Im}\left[q_{m}\right]\right)\right\}
\end{gathered}
$$

where $\left\{P_{k}\right\}$ is feed forward filter taps and $\left(q_{m}\right)$ is feed beck filter taps i $\mu$ and $v$ are ajaptation rate and $S_{n}$ is input vector,$a_{n}$ is previous detected data In their work, channel was time varying and their modification showed to be effective to cope with non-minimum phase channel

3-HARRIS [19] proposed a new version of an LMS algorithm which implemented different variable adaptation rate for each weight of equalizer.

4Weeb \& Steel [43] proposed an alternative system which they call it Rake-type equalizer to control LMS-decision feed back equalizer and improve it's tracking properties by preventing it from going to error propagation.

5-Mathews [32] developed a variable step size LMS algorithm in which step size changes in proportion to the negative of the gradient of the squared estimation error with respect to the convergeace parameter.

6-Shan \& Kailath [42] proposed an adaptive gain control scheme for adaptive equalizers, which uses the time average of the gradient estimate to control the step size 


$$
\begin{gathered}
w_{k+1}=w_{k}+g_{k} \varepsilon_{k} X_{k} \\
g_{k+1}=\alpha p_{k}
\end{gathered}
$$

where $X_{k}$ is input vector and $g_{k}$ is adaptive gain at instant $k$ and $p_{k}$ is correlation factor which is determined as

$$
\rho_{k}=\lambda \rho_{k-1}+(1-\lambda) \overline{x_{k}} \varepsilon_{k}
$$

In this equation $\lambda$ is a forgetting factor close to 1 and

$$
\bar{x}_{k}=\frac{1}{M} \sum_{j=1}^{M} \alpha_{k}^{j}
$$

where $M$ is input vector dimension and $\alpha_{k}^{j}$ is jth element of input vector at time instant k.

7-J. Chao [3] introduces a new adaptive filter algorithm using eigenvalue reciprocals as step size. 


\section{Chapter 3 \\ System Description}

\subsection{SYSTEM MODEL:}

In this chapter the overall system model and it's components are explained. The basebend model of the system under investigation is shown in figure 3.1.

\subsection{MODULATION:}

The modulation scheme is assumed to be quadrature phase shift keying (QPSK). The transmitted symbols are complex zero mean and mutually uncorrelated.

where $\zeta(n)$ denotes delta function

$$
\begin{gathered}
E\left[a_{k}\right]=0 \\
E\left[a_{k} a_{n}^{*}\right]=\sigma_{a}^{2} s(n)
\end{gathered}
$$

$$
\zeta(n)= \begin{cases}1 & n=0 \\ 0 & n \neq 0\end{cases}
$$

$E[$.$] is the expectation operator and \sigma_{a}^{2}$ is the variance of the data which is set to unity.

\subsection{DATA STRUCTURE:}

It is known that using periodic known training sequences is appropriate for digital mobile communication [8] and frame by frame signalling is basically what is proposed for the narrow bani iDMA digital communication radio system for north America [13].

In this strategy each frame is comprised of an initial training sequence of length $\mathrm{Nt}$ symbols followed by a data sequence of length $\mathrm{Nd}$ symbols so there are $\mathrm{N}=\mathrm{Nt}+\mathrm{Nd}$ symbols in each transmitted frame.

In our simulation we have used the same structure of alternating training and data sequence as shown in Fig(3.3) and since our main concern is to evaluate the equalizer's performance in fading environment, we have not accuunted for signalling information such as: guard and ramp times; synchronization symbols and other control signals which would occur at the beginning of each time slot. 


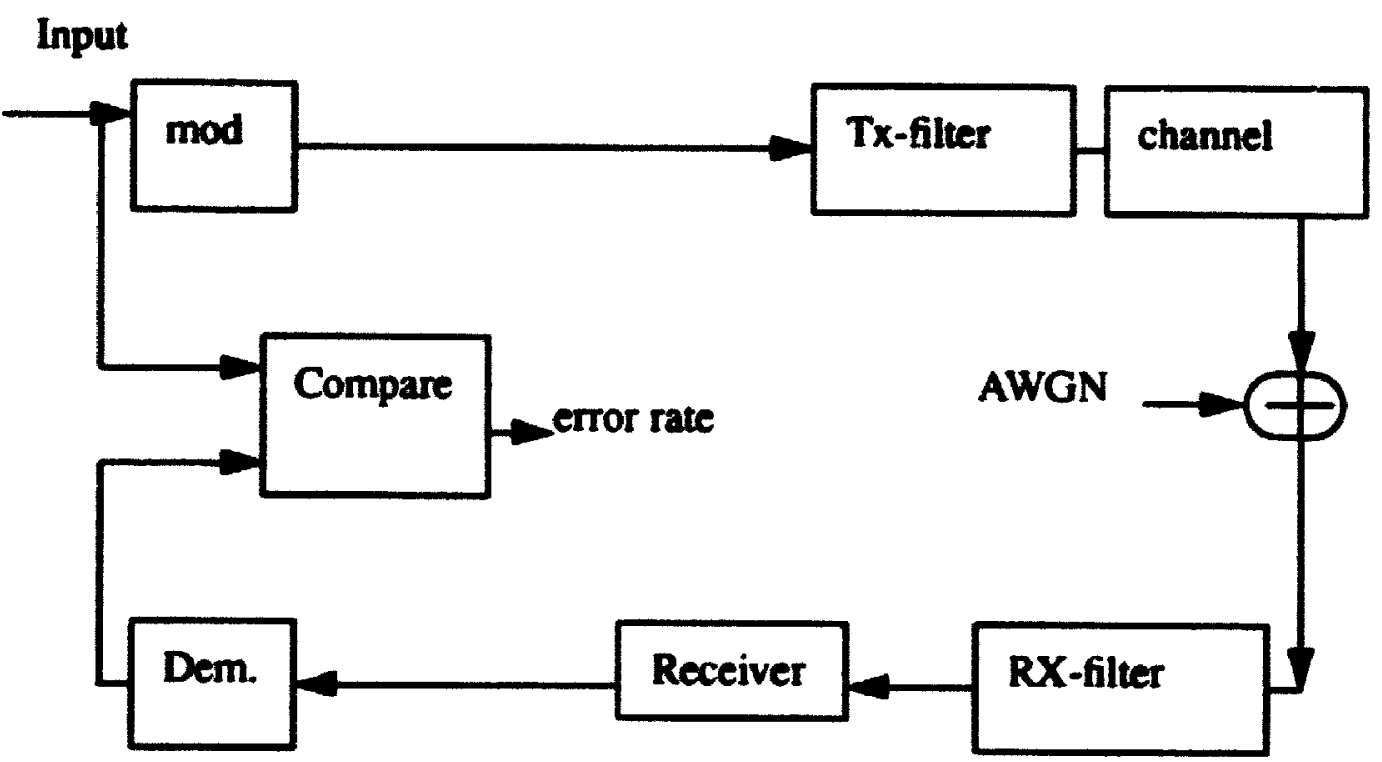

Figure (3.1) System model 


\subsection{TRANSMIT AND RECEIVE FILTERS:}

We used square root raised cosine filter with roll off factor equal to 3.

The frequency response of a square root raised cosine filter, $H(f)$ is given by the following equation and shown in Fig (3.2).

$$
H(f)=\left(\begin{array}{cc}
1 & |f| \leq \frac{1}{2 T}(1-\alpha) \\
\sin \left[-\frac{T}{4 \alpha}\left(2 \pi|f|-\frac{\pi}{T}(1+\alpha)\right)\right] & \frac{1}{2 T}(1-\alpha)<|f| \leq \frac{1}{2 T}(1+\alpha) \\
0 & |f|>\frac{1}{2 T}(1+\alpha)
\end{array}\right.
$$

$\alpha$ is the roll-off factor of the filter which is between 0 and 1.

The over all transfer function of two square root raised cosine filters has the raised cosine characteristic.

$$
H_{R C}(f)=\left(\begin{array}{cl}
1 & |f| \leq \frac{1}{2 T}(1-\alpha) \\
\frac{1}{2}\left[1-\sin \left[\frac{T}{2 \alpha}(2 \pi \mid f)-\frac{\pi}{T}\right]\right] & \begin{array}{c}
\frac{1-\alpha}{2 T}<|f| \leq \frac{1+\alpha}{2 T} \\
0
\end{array} \\
|f|>\frac{1}{2 T}(1+\alpha)
\end{array}\right.
$$

Obviously this transfer function meets the Nyquist criteria for zero ISI [38].

\subsection{THE NOISE CHARACTERISTICS:}

The noise is assumed to be Additive White Gaussian Noise (AWGN).

It has a two-sided power spectral density of No :

$$
E[Z(t)]=0
$$




$$
E\left[Z(t 1) Z^{*}(t 2)\right]=\operatorname{Nos}(t 1-t 2)
$$

where $\zeta()$ is delta function

$$
\zeta(x)= \begin{cases}1 & x=0 \\ 0 & x \neq 0\end{cases}
$$




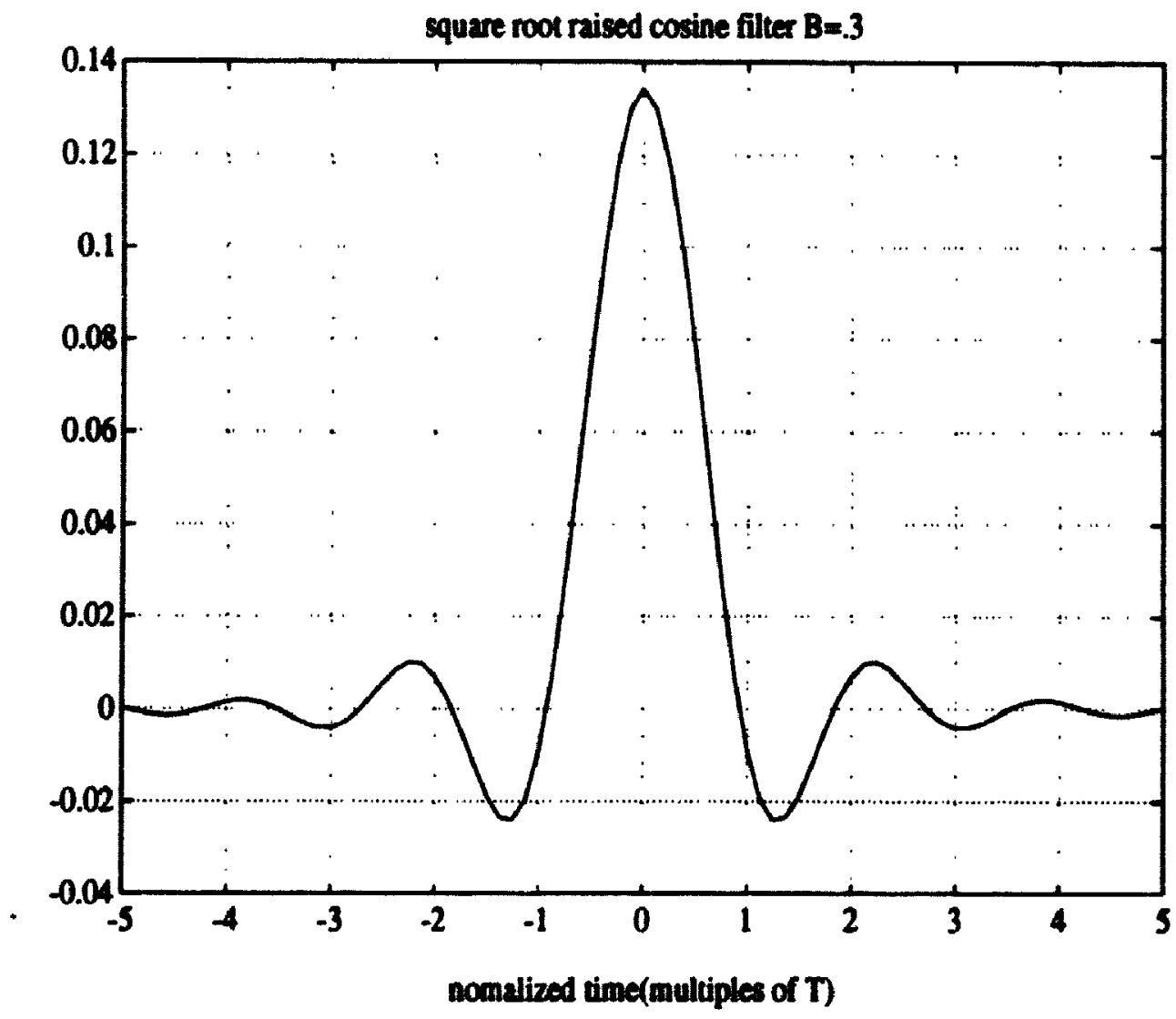

Figure (3.2) Square root raised cosine fitter 


\begin{tabular}{|l|l|l|l|}
\hline $\begin{array}{l}\text { training } \\
\text { sequence }\end{array}$ & data sequence & $\begin{array}{l}\text { training } \\
\text { sequence }\end{array}$ & data sequence \\
\hline
\end{tabular}

Figure (3.3) Signalling format 


\subsection{THE CHANNEL MODELS:}

In this thesis, the model used to simulate fading channel is the one which has been known as sum of sines model $[24,2,30]$.

In this model, each coefficient gm(kt) of the complex-valued base-band channel is given by the following equation:

$\mathrm{kT}=\mathrm{is}$ the discrete time variable

$$
g m(k t)=\sum_{n=0}^{N_{s}} g m_{n} \cdot \cos \left(2 \cdot \pi \cdot \zeta_{n} \cdot f_{d m} \cdot k \cdot T\right)
$$

$g m_{n}=$ is the complex valued amplitude for the $n$th Doppler frequency

$f_{d m}=i s$ the maximum Doppler frequency

$\zeta_{n}=$ is the relative frequency scaling factor for the nth Doppler frequency

$\mathrm{Ns}+1$ is the number of weighted sinusoids. The relative frequency scaling factor for the nth Doppler frequency is:

$$
S_{n}=\cos (2 \cdot \pi \cdot n / N)
$$

with $n=0$ corresponding to the maximum Doppler frequency

The in-phase and quadrature components of the amplitude for the nth Doppler frequency are respectively:

$$
\begin{gathered}
\operatorname{RE}\left(\mathrm{gm}_{\mathrm{n}}\right)=\left\{\begin{array}{cc}
\sqrt{2} \cdot \cos (\pi / 4) & n=0 \\
2 \cdot \cos \left(\pi \cdot \mathrm{n} / \mathrm{N}_{s}\right) & 1 \leq \mathrm{n} \leq \mathrm{N}_{s}
\end{array}\right. \\
\operatorname{Im}\left(\mathrm{gm}_{\mathrm{n}}\right)=\left\{\begin{array}{cc}
\sqrt{2} \cdot \sin (\pi / 4) & \mathrm{n}=0 \\
2 \cdot \sin \left(\pi \cdot \mathrm{n} / \mathrm{N}_{\mathrm{s}}\right) & 1 \leq \mathrm{n} \leq \mathrm{N}_{\mathrm{s}}
\end{array}\right.
\end{gathered}
$$

with the sum of sines method, the use of a larger number of weighted sinusoids lead to a more accurate Rayleigh fading but in has been shown that Rayleigh fading can be accurately simulated with by just nine (Ns-9) discrete sinusoids 2] . 
The sum of sines model is used to simulate individual rays. In order to represent a multipath model with a large delay spread we used (i) two-ray and (ii) three-ray models. The two ray model is shown in Figure (3.4). It consists of two rays of equal average power separated by a full symbol duration $T$. The two rays are individually Rayleigh and statistically independent. The three-ray model consists of three rays spaced by half the symbol duration (T/2), and have a [ $0-5-15]$ dB power profile as shown in Figure (3.6). The two-ray channel is a non-minimal phase channel, which is hard to equalize, while the three-ray model is an easier (and more realistic) channel model. 


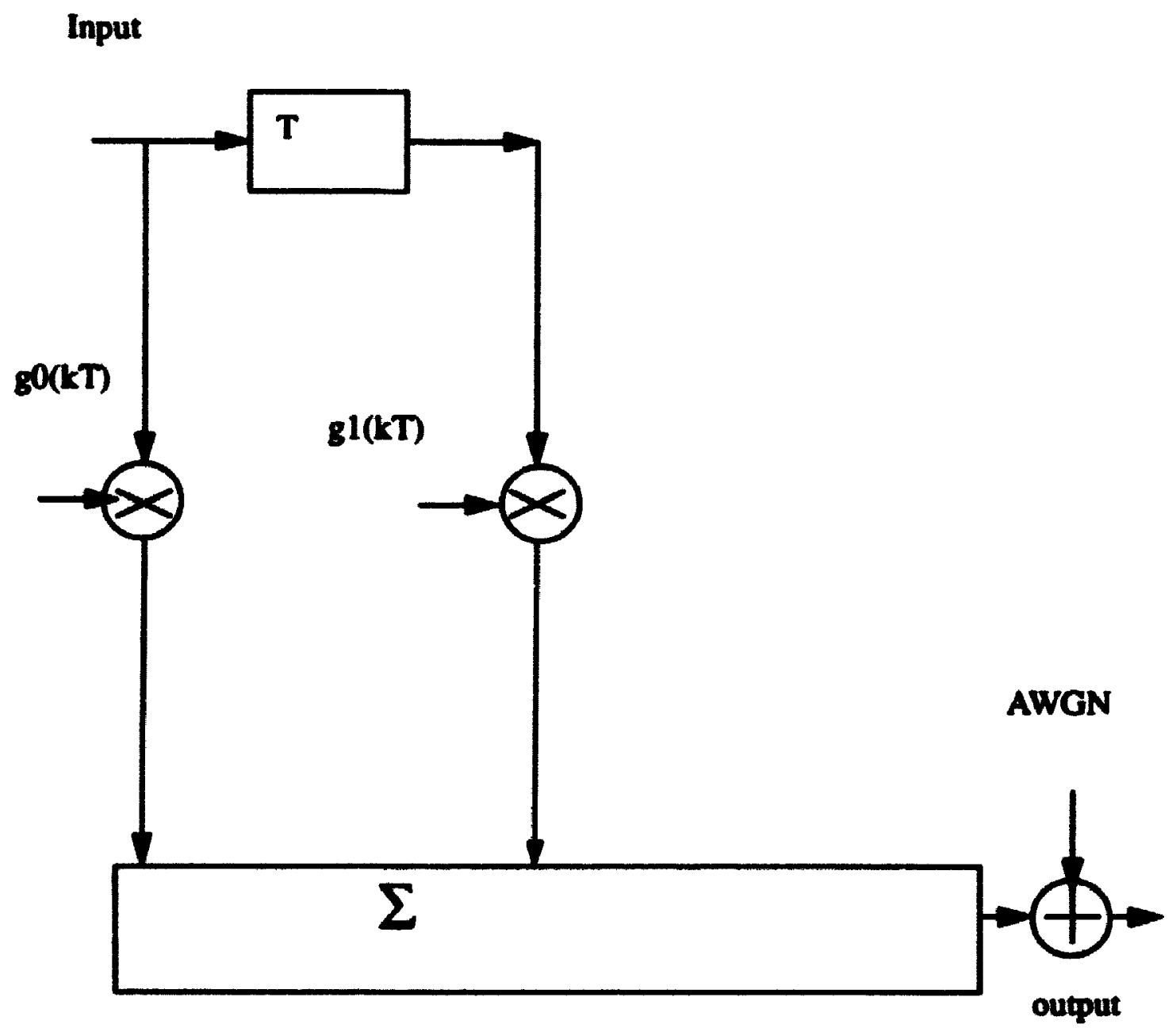

Figure (3.4) T-epaced tapped delay line model for a 2-ray multipath fading channel. 
23

relative power

o

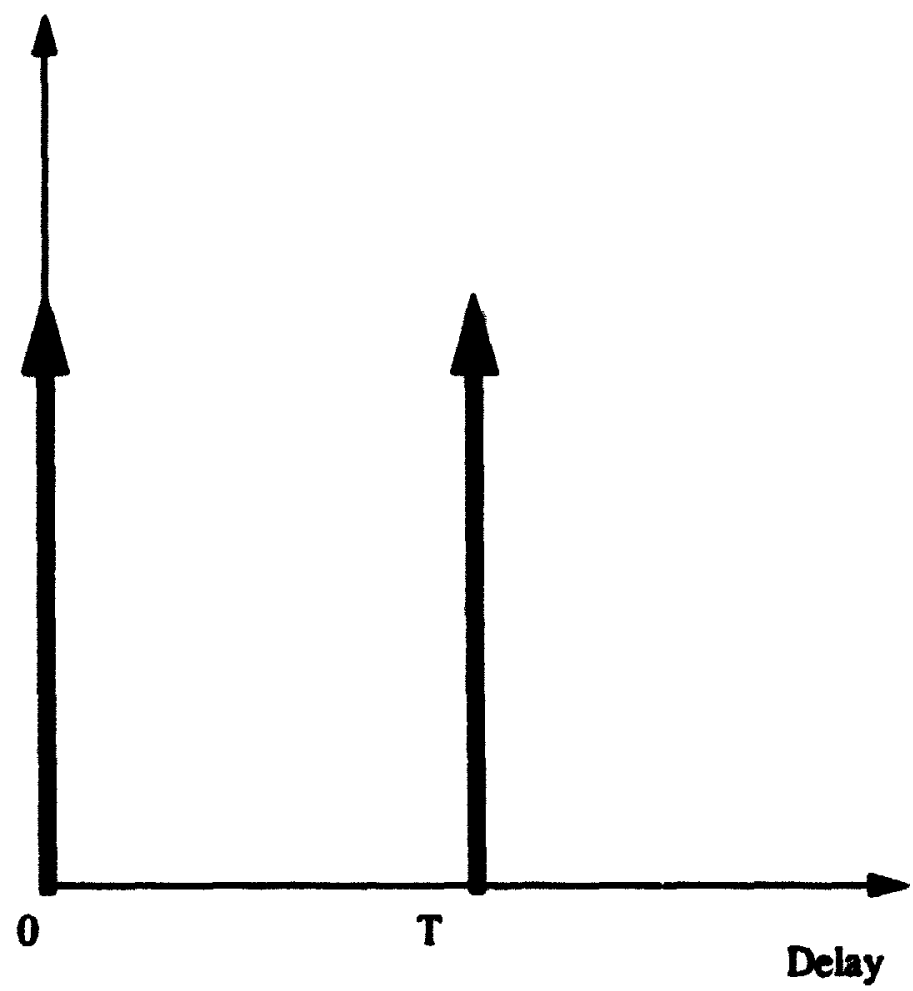

$-15$

[dB]

Figure (3.5) 2 equal power ray fading channel 
Input

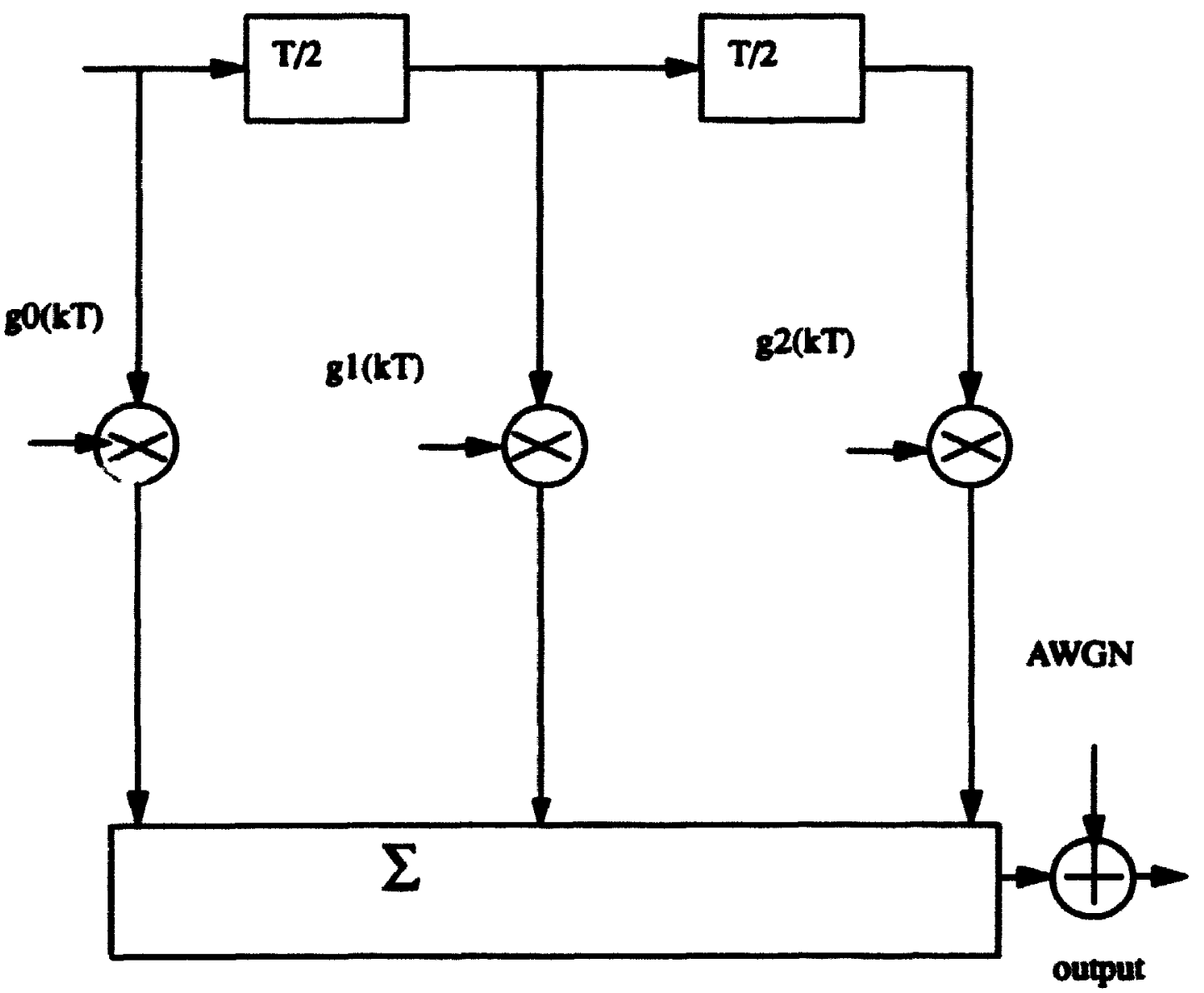

Flare (3.6) T/2-epaced tapped Delay Line model for a 3-ray multipath fading cluanad 
25

relative power

$\mathbf{0}$

$-5$

[dB]

$-15$

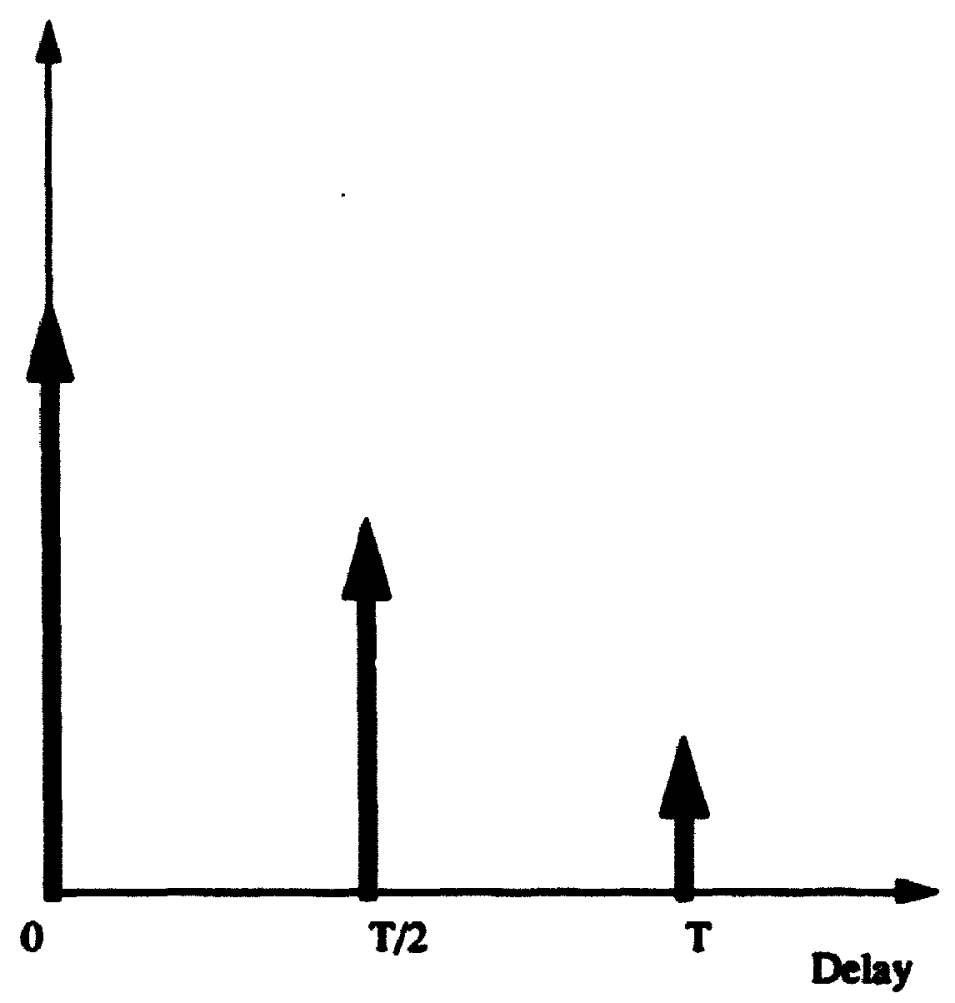

Figure (3.7) 3-ray Multipath foding channed 


\subsection{RECEIVER STRUCTURE:}

The receiver consists of a decision feedback equalizer plus a decision device.

Digital realization of Decision-Feedbeck equalizer has been used for channel equalization. This means that the received signal at the input of the equalizer is sampled at a rate of $M / T$ where $M$ is an integer greater than or equal to one. The tap spacing of the forward filter is $T M$. The general structure of the equalizer is shown in Fig (3.9).

The DFE is comprised of a feed forward transversal filter. Wf , which is escentially a linear equalizer and a feedbeck filter Wb. The forwand filer removes the precursor ISI so that zero crossings occur at all samples of the received signal prior to the main lobe at the current sample instant. The postcursor ISI at the samples after the main lobe of the received signal response can be eliminated by subureting the feedbeck-filtered detected signal from the output of the forward filter.

In all cases the number of forward taps is split to $\mathrm{Ll}$ future taps operating on precursor ISI and L2 past taps openting on post-cursor ISI and one central tap.

The output of the feedbeck filter is

$$
F B=\sum_{m=1}^{L_{b}} f_{m} \times a_{n-m-D}
$$

where $f_{m}$ is the $m^{\text {th }}$ feedback tap coefficient and $D$ is the delay in the equalizer which also determines the position of the central reference tap and ' $\mathrm{a}_{n-m}$ ' is the (n-m)th decision feed beck filter's ouput, $L_{b}$ is the number of taps in decision feed beck filter.

The output of forward filter can be written as

$$
F F=\sum_{m=0}^{L} c_{m} \times r(n T)
$$

where $c_{m}$ is the moth forward tap coefficient at $t=n T$ and $\mathrm{r}(\mathrm{nT})$ is the input sample at time instant tin $n$ and $L_{f}$ is the total number of forward taps .

The input of decision device will then be 


$$
Y_{n}=\sum_{m=1}^{L_{h}} c_{m} \times r(n T)+\sum_{m=1}^{L_{i}} f_{m} \times a_{n-m-D}
$$

Based on $Y_{n}$ the decision device will decide which one of the four possible symbols was transmitted

$$
a_{n}=\frac{1}{\sqrt{2}}\left\{\operatorname{sgn}\left[\operatorname{Re}\left(Y_{n}\right)\right]+j \operatorname{sgn}\left[\operatorname{Im}\left(Y_{n}\right)\right]\right\}
$$

where

$$
\operatorname{sgn}(x)= \begin{cases}1 & ; x \geq 0 \\ -1 & ; x \geq 0\end{cases}
$$
$\operatorname{RE}(\bullet)$ is the real part of $(\bullet)$ and $\operatorname{Im}(\bullet)$ is the imaginary part of $(\bullet)$. The factor $\frac{1}{\sqrt{2}}$ is used
to normalize data variance. 


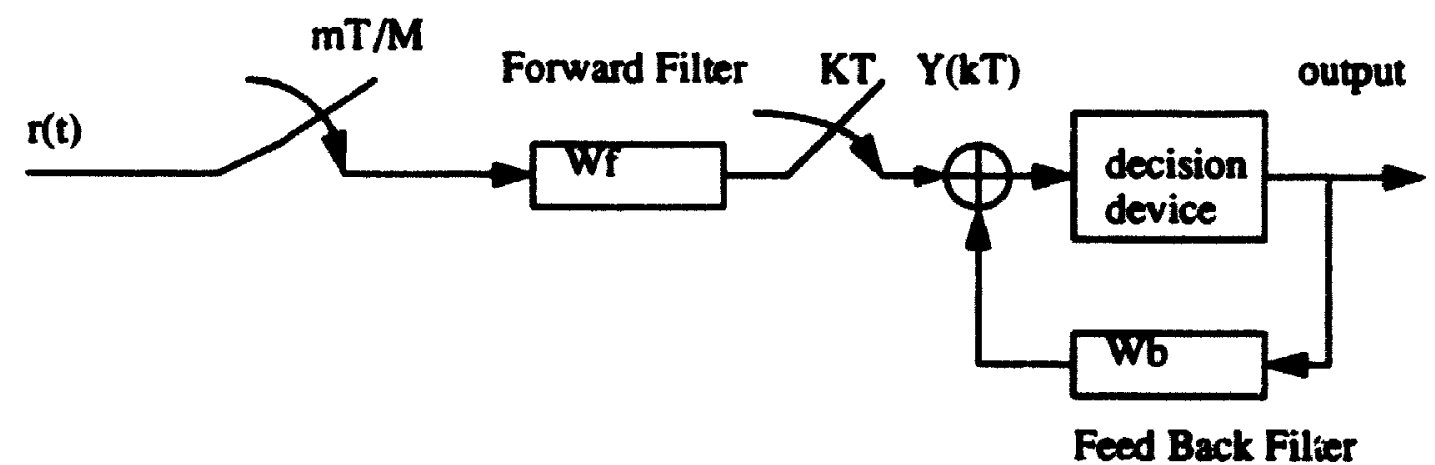

Fig (3.8) T/M-Spaced Decision-Feedback Equalizer 


\subsection{DESCRIPTION OF THE SIMULATION PROGRAM:}

A program was written to investigate the performance of a digital mobile communication system with R.B.F equalizer. The Rayleigh fading of the complex valued tap coefficient is simulated using the sum of sines model. The fade rate of the tap coefficient is determined by the maximum Doppler frequency which is an input parameter to the simulation. Each complex experiment simulates an ensemble of independent trials of digital communication channel.

Channel has been initialized at the beginning of each trial by assigning a set of 18 independent random phase with uniform distribution to 18 sine terms (real and imaginary part) in sum of sines models, time evolution of channel is deterministic based on sum of sines model during the rest of frame until the beginning of the next trial when the channel is initialized again.

In order to simplify the simulation, the total energy per transmitted symbol at the channel input has been normalized to unity, and the transmitter and receiver filters are set to have unit energy each. 


\section{Chapter 4 \\ Simulation Results and Performance Analysis}

\subsection{INTRODUCTION:}

In this chapter three different forms of the radial basis function (R.B.F) equalizer are studied. A simple form of R.B.F algorithm with a single Gaussian term is investigated in section 4.2. The general R.B.F algorithm or Block R.B.F is analyzed and compared with the LMS and RLS algorithm in section 4.3, and the modified block R.B.F algorithm is introduced and analyzed in section 4.4 .

\subsection{R.B.F ALGORITHM WITH SINGLE GAUSSIAN TERM:}

In this section we present the analysis of RBF equalizer and show its superiority over the LMS equalizer. The analysis is conducted using a single Gaussian term as cost function. The R.B.F cost function that should be minimized is

$$
T=\exp \left(\left|\left(y_{d}-y_{k}\right)\right|^{2} /\left(2 \cdot \sigma^{2}\right)\right)
$$

In this expression, $y_{k}$ is the equalizer output at time $k_{;} y_{d}$ is the desired output at the same instant and $\sigma$ is the cluster width.

The relation between the input and output of the equalizer is:

$$
y_{k}=x_{k}^{T} \cdot W
$$

$y_{k}$ is the equalizer output, $W$ is a weight vector, $X_{k}$ is the input vector and $T$ is the transpose operator. For this cost function the gradient descent updating equation is :

$$
w_{k+1}=w_{k}+\left(\beta /\left(2 \cdot \sigma^{2}\right)\right) \cdot\left(y_{d}-y_{k}\right) \cdot \exp \left(\left|\left(y_{d}-y_{k}\right)\right|^{2} /\left(2 \cdot \sigma^{2}\right)\right) x_{k}^{*}
$$

where $W_{k}$ is the weight at the $k^{\text {th }}$ iteration.

To compare this equation with the LMS updating equation which is

$$
w_{k+1}=w_{k}+\beta \cdot\left(y_{d}-y_{k}\right) \cdot x_{k}^{*}
$$


we define

$$
\beta_{e q}(k)=\left(\beta /\left(2 \cdot \sigma^{2}\right)\right) \cdot \exp \left(\left|y_{d}-y_{k}\right|^{2} /\left(2 \cdot \sigma^{2}\right)\right)
$$

or

$$
\beta_{e q}(k)=\beta_{\min } \cdot \exp \left(\left|y_{d}-y_{k}\right|^{2} /\left(2 \cdot \sigma^{2}\right)\right)
$$

The updating equation for radial basis function equalizer becomes:

$$
w_{k+1}=w_{k}+\beta_{e q}(k) \cdot\left(y_{d}-y_{k}\right) \cdot x_{k}^{*}
$$

where $\beta_{\min }$ is equal to $\beta /\left(2 \sigma^{2}\right)$.

Eq. (4.6) indicates that the RBF algorithm can be looked at as a variable step size LMS algorithm and it can be analyzed using a procedure similar to the one used in [19][26].

Let $W_{\text {opt }}$ be the optimum weight vector (Weiner solution), we analyze the convergence behavior of the mean weight vector toward this optimum weight.

Assumption(I): Independent input vector

Let the input vector be $X_{k}$ and the desired scalar output be $y_{d}$ then,

$$
Y_{d}=X_{k}^{T} \cdot w_{o p t}+e
$$

where "e " is a zero mean Gaussian process. The input process $\mathbf{X}_{k}$ is assumed to be an independent sequence.

This independence assumption is of course far from true, but it has been shown in [33] that final predictions made using it are in excellent agreement with experiments and simulations.

Asoumption(II): $\beta_{e q}(k)$ can be replaced by its expected value $E\left[\beta_{e q}(k)\right]$ and

$$
E\left[\beta_{e q}(k)\left(\left(y_{k}-y_{d}\right) X_{k}^{*}\right)\right]=E\left[\beta_{e q}(k)\right] \cdot E\left[\left(y_{k}-y_{d}\right) \cdot x_{k}^{*}\right]
$$

This assumption is true if $\beta_{e q}(k)$ is either a constant or independent of both the error and the input sequence, both conditions do not apply to our model. But the assumption is approximately true when the error is small according to the following argument: 
we notice that the left side of equation 4.8 can be written as:

$$
\begin{gathered}
E\left[\beta_{e q}(k)\left(y_{k}-y_{d}\right) X_{k}^{*}\right]=E\left[\beta_{e q}(k)\right] E\left[\left(y_{d}-y_{k}\right) x_{k}^{*}\right]+ \\
E\left[\left(\beta_{e q}(k)-E\left[\beta_{e q}(k)\right]\right) \cdot\left(y_{d}-y_{k}\right) \cdot x_{k}^{*}\right]
\end{gathered}
$$

and $\beta_{e q}(k)$ can be written as

$$
\beta_{e q}(k)=\left(\beta /\left(2 \cdot \sigma^{2}\right)\right)+\left(\left(\beta \cdot\left|y_{d}-y_{t}\right|^{2}\right) /\left(4 \cdot \sigma^{4}\right)\right)
$$

The second term in eq. (4.10) is very small and one can say $\beta_{e q}(k)$ is varying slowly around it's mean value. Therefore, the second term in $\propto q$. (4.9) is negligible. This will justify our second assumption and will allow us to derive theoretical results which can be verified by simulations.

\subsubsection{STABILITY CONDITION:}

Let $R$ be the input vector covariance matrix

$$
R=E\left[X_{k}^{*} \cdot\left(X_{k}\right)^{T}\right]
$$

recalling that $y_{d}$ was equal to $X_{k}{ }^{T} \cdot W_{\text {opt }}+e$ our averaged updating equation can be written as:

$$
E\left[w_{k+1}\right]=E\left[w_{k}\right]+E\left[\beta_{e q}\right] \cdot E\left[X_{k}^{*}\left[X_{k}^{T} \cdot w_{o p t}-\left(x_{k}^{T} \cdot w_{k}\right)\right]\right]
$$

or

$$
E\left[w_{k+1}\right]=E\left[w_{k}\right]-E\left[\beta_{e q}\right] \cdot R \cdot E\left[w_{k}-w_{o p t}\right]
$$

by detining an error weight vector as: $w_{k}^{e}=w_{k}-w_{o p t}$

$$
\begin{gathered}
E\left[W_{k+1}^{e}\right]=E\left[W_{k}-W_{o p t}\right]-E\left[\beta_{e q}(k)\right] \cdot R \cdot E\left[W_{k}-W_{o p t}\right] \\
E\left[W_{k+1}^{e}\right]=E\left[W_{k}^{e}\right]-E\left[\beta_{e q}(k)\right] \cdot R \cdot E\left[W_{k}^{e}\right]
\end{gathered}
$$




$$
E\left[W_{k+1}^{k}\right]=\left[I-E\left[\beta_{e q}(k)\right] \cdot R\right] \cdot E\left[W_{k}^{e}\right]
$$

where $I$ is the identity matrix. This equation leads to a stable solution if and only if

$$
\prod_{k=0}^{n}\left[I-E\left[\beta_{e q}(k)\right] \cdot R\right] \rightarrow 0
$$

by this condition $E\left[W_{k}^{e}\right] \rightarrow 0$ or $W_{k}$ converge to $W_{o p t}$ and a sufficient and necessary condition for this equation to hold is [44]

$$
E\left[\beta_{e q}(k)\right]<2 / \lambda_{\max }
$$

or

$$
E\left[\left[\beta /\left(2 \cdot \sigma^{2}\right)\right] \cdot \exp \left(\mid y_{k}-y_{d} /\left(2 \cdot \sigma^{2}\right)\right)\right]<2 / \lambda_{\max }
$$

where $\lambda_{\max }$ is the largest eigenvalue of the covariance matrix, $R$.

By comparing this condition with that for LMS equalizer which is:

$$
\beta<2 / \lambda_{\max }
$$

We may conclude that the R.B.F equalizer can converge much faster than the LMS equalizer. The reason is that the stability condition of the RBF applies to the average value of the adaptation rate, therefore the maximum value for the RBF step size could be much larger than the step size of the LMS.

In Appendix B the stability condition for R.B.F algorithm in a time variant environment is investigated . 


\subsubsection{CONVERGENCE RATE:}

In this sub-section we derive the convergence speed of the average weight toward its optimum value.

Equation (4.16) is an uncoupled homogeneous difference equation, and can be solved as in [44]:

$$
E\left[\hat{W}^{e}(k)\right]=\left(I-E\left[\beta_{e q}(k)\right] \cdot \Lambda\right)^{k} \cdot W^{e}(0)
$$

Where $\Lambda$ is the modal matrix of the input data auto correlation matrix $R, \hat{W}^{e}(k)$ is an error vector in primed coordinates and $W^{e}(0)$ is an initial value for error vector.

The geometric ratio for the $\mathrm{p}^{\text {th }}$ coordinate is

$$
r_{p}(k)=\left(1-E\left[\beta_{e q}(k)\right] \lambda_{p}\right)
$$

where $\lambda_{p}$ is the $p^{\text {th }}$ eigenvalue of $R$.

By fitting an exponential envelope to the geometric sequence of Eq. (4.21), it can be concluded that the average convergence time $\left(\tau_{p}\right)$ is approximately [44]:

$$
\begin{aligned}
r_{p}(k) & =1-\frac{1}{\tau_{p}(k)} \\
\tau_{p}(k) & =\frac{1}{E\left[\beta_{e q}(k)\right] \lambda_{p}}
\end{aligned}
$$

Comparing this time cunstant with that for LMS equalizer, which is

$$
\tau=\frac{1}{\beta \lambda_{p}}
$$

we conclucie that for a given steady state error level, (or $\beta$ min $\left(\beta_{\text {eq }}\right)$ ) the proposed equalizer at each step is converging faster than the fixed rate LMS equalizer (the average value of $\beta_{e q}(k)$ is larger than or equal to it's minimum value) or $\tau_{p}(k) \leq \tau$. 


\subsubsection{STEADY STATE BEHAVIOR ANALYSIS:}

This analysis is basically the same as [22]. In this thesis it has been modified for radial basis function equalizer and in order to make analysis tractable, it has been limited to linear structure.

In Appendix ' $A$ ', the excess MSE and misadjustment factor for time invariant environment have been computed in terms of average value and second order moment of equivalent adaptation rate. These moments can be expressed in terms of variance of cluster and $\boldsymbol{\beta}_{\text {min }}$.

The misadjustment factor is defined as [44]:

$$
M=\frac{\text { average - excess - error }}{\text { err }_{\min }}
$$

Excess mean square error is caused by random noise in the weight vector. This noise is produced by the gradient estimation noise which is inherent in all stochastic gradient based algorithms.

In appendix ' $A$ ' it is shown that the steady state mean square error can be written as:

$$
\operatorname{MSE}(\infty)=\frac{e r r_{\min }}{1-\sum_{i=1}^{n} \frac{\mu \cdot \lambda_{i}}{-\mu \cdot \lambda_{i}+\bar{\beta}}}
$$

and that the misadjustment factor is computed as :

$$
M=\frac{M S E(\infty)-e r r_{\text {min }}}{e r r_{\text {min }}}
$$

or

$$
M=\frac{\sum_{i=1}^{n} \frac{\mu \cdot \lambda_{i}}{-\mu \cdot \lambda_{i}+\bar{\beta}}}{1-\sum_{i=1}^{n} \frac{\mu \cdot \lambda_{i}}{-\mu \cdot \lambda_{i}+\bar{\beta}}}
$$


In these expressions $\bar{\beta}$ is the statistical expectation of $\beta_{e q}$ and $\mu$ is the statistical expectation of $\beta_{e q}^{2}, \lambda_{i}$ is the $i^{\text {th }}$ eigenvalue of input data covariance matrix and $e \pi_{\min }$ is expected value of $\left(e^{2}\right)$

The steady state error level for the R.B.F algorithm in a time variable environment is derived in appendix $\mathbf{C}$. It has been shown that the steady state mean square error can be expressed by the following equation:

$$
M S E(\infty)=\frac{e r r_{\min }}{1-\sum_{i=1}^{n} \frac{\mu \cdot \lambda_{i}}{-\mu \cdot \lambda_{i}+\bar{\beta}}}+\frac{\sum_{i=1}^{n} \frac{\sigma_{z}^{2}}{\bar{\beta}-\mu \cdot \lambda_{i}}}{1-\sum_{i=1}^{n} \frac{\mu \cdot \lambda_{i}}{-\mu \cdot \lambda_{i}+\bar{\beta}}}
$$

In this expression, $\bar{\beta}$ is the average aduptation rate , $\mu$ is the second order moment of the adaptation rate, $\sigma_{z}^{2}$ is the power of the Gaussian random process which was added to the channel tap at each step to model the non-stationarity inside the channel. $\lambda_{i}$ is the $i^{\text {th }}$ eigenvalue of the input data covariance matrix. 


\subsubsection{SIMULATION RESULTS:}

In this section the radial basis function algorithm's performance in fixed and time variable channels has been studied by simulations [4.1-4.12].

In the first set of simulation tests Fig (4.2-4.7), the channel model consists of 2, Tspaced, equal power independent Rayleigh fading rays. The R.B.F equalizer is adapted to estimate the channel, and the criterion used to judge the estimation accuracy is the mean square error (MSE) between the output of the channel and the equalizer's output [4.1].

In Figures 4.2 and 4.3 the channel was kept constant during the test. The results in these figures represent the MSE averaged over 100 independent tests. In figure 4.2 the noise variance is small (.01). The range of the adaptation step sizes varies from a minimum of .01 to a maximum of .4. The MSE of the R.B.F equalizer shrinks rapidly from its high initial value down to its lo vest possible value of .01 (the noise variance). This behavior is compared to the LMS convergence on the same graph. Two LMS fixed step sizes are used : .01 and .4 . In the case of a small step size, the LMS converges very slowly but reaches the same minimum value of .01 . In the case of a large step size, the LMS converge fast but its minimum MSE (MMSE) is high. Figure 4.3 gives the similar results for higher variance (.03).

Figures 4.4 to 4.6 demonstrate R.B.F and LMS channel estimator at three different Doppler frequencies 2 and 10 and $20 \mathrm{~Hz}$.

From these figures one can conclude that the RBF algorithm has better performance than LMS algorithm in fading channel with Doppler frequency up to $20 \mathrm{~Hz}$. For channel variation rate above $20 \mathrm{~Hz}$, RBF is acting similar to LMS algorithm with step size equal to its maximum rate.

In Fig 4.7 we tried to demonstrate how responsive R.B.F algorithm is to any sudden fluctuation in channel in comparison to the LMS algorithm. In this case for the same level of steady state error RBF estimator and LMS estimator are compared; channel has been updated every $\mathbf{5 0 0}$ symbols by adding a Gaussian random variable with variance equal to .01 to channel taps. 
This simulation test shows that, for a given steady state error level, convergence rate is increased by almost 50\% in R.B.F compare to LMS algorithm.

In the second set of simulation tests (Figures 4.8-4.14) we test the channel equalization mode. The channel model is the same as in the first set of simulations, but now the equalizer tups are adapted to minimize the difference between the equalizer outputs and the desired QPSK data values. The equalizer consists of 3 T-spaced forward taps and one feedbeck tap.

In figures 4.8 and 4.9 two LMS equalizers with different edaptation rates in fading channel with Doppler frequency equal to zero are compered with R.B.F equalizer. The curves in figure 4.8 are derived for $S / N$ equal to $20 \mathrm{~dB}$ and figure(4.9) is based on $S / N$ equal to $15 \mathrm{~dB}$.

In Figures 4.10 to 4.12, R.B.F equalizer and LMS equalizer in different Doppler frequencies at $\mathrm{S} / \mathrm{N}=20 \mathrm{~dB}$ are compared.

In Figure 4.13, R.B.F equalizer's performance in terms of training period and related symbol error rate at $S / N$ equal to $2 C \mathrm{~dB}$ is demonstrated and compared with LMS equalizer.

In Figure 4.14, LMS and R.B.F equalizers with the same steady state error level are compared in terms of symbol error rate versus $S / N$ at Doppler frequency equal to $5 \mathrm{~Hz}$.

These figures show that the RBF equalizer improve the trade off between the steady state error level and convergence speed significantly .

Simulation shows that, for low signal to noise ratio, the performance of R.B.F and LMS algorithm, for a given level of steady state mean square error, is almost the same. R.B.F algorithm is slightly worse than LMS algorithm. The reason for this phenomenon is the fact that we set the noise variance factor in R.B.F algorithm at a fixed value which was optimum at signal to noise ratio equal to $15 \mathrm{~dB}$ [equal to noise variance]. Due to this fixed variance factor, at low signal to noise ratio the value of Gaussian function argument is always high and has not been scaled properly (divided by small value in compare to noise variance), therefore step size remains close to it's upper limit at low signal to noise ratio most of the time. 


\subsubsection{COMPUTATIONAL COMPLEXITY:}

As it has been mentioned in chapter 2. the number of complex multiplications in LMS equalizer for each input is $2 \mathrm{~N}+1$ operations where $\mathbf{N}$ is the total number of equalizer's caps [feed forward and feedback].

In order to compare the LMS equalizer complexity with the R.B.F equalizer's complexity , we assume Gaussian function can be approximated with the first two terms of it's Taylor series expansion.

By assuming

$$
\exp \left(\mid e r r^{2} /\left(2 \sigma^{2}\right)\right)=1+k \mid e r h^{2}
$$

$k$ is a fixed integer.

The number of complex multiplications for R.B.F equalizer will be $2 \mathrm{~N}+2$. One extra multiplication is coming from computing lerr $\left.\right|^{2}$ which is $e r r \times(e r r)^{*}$ and the rest of operations are the same as the LMS equalizer.

There is an extra complexity in the R.B.F equalizer due to controlling it's stability. In other words; adaptation rate has to be compared with it's limit imposed by stability condition at each step. 


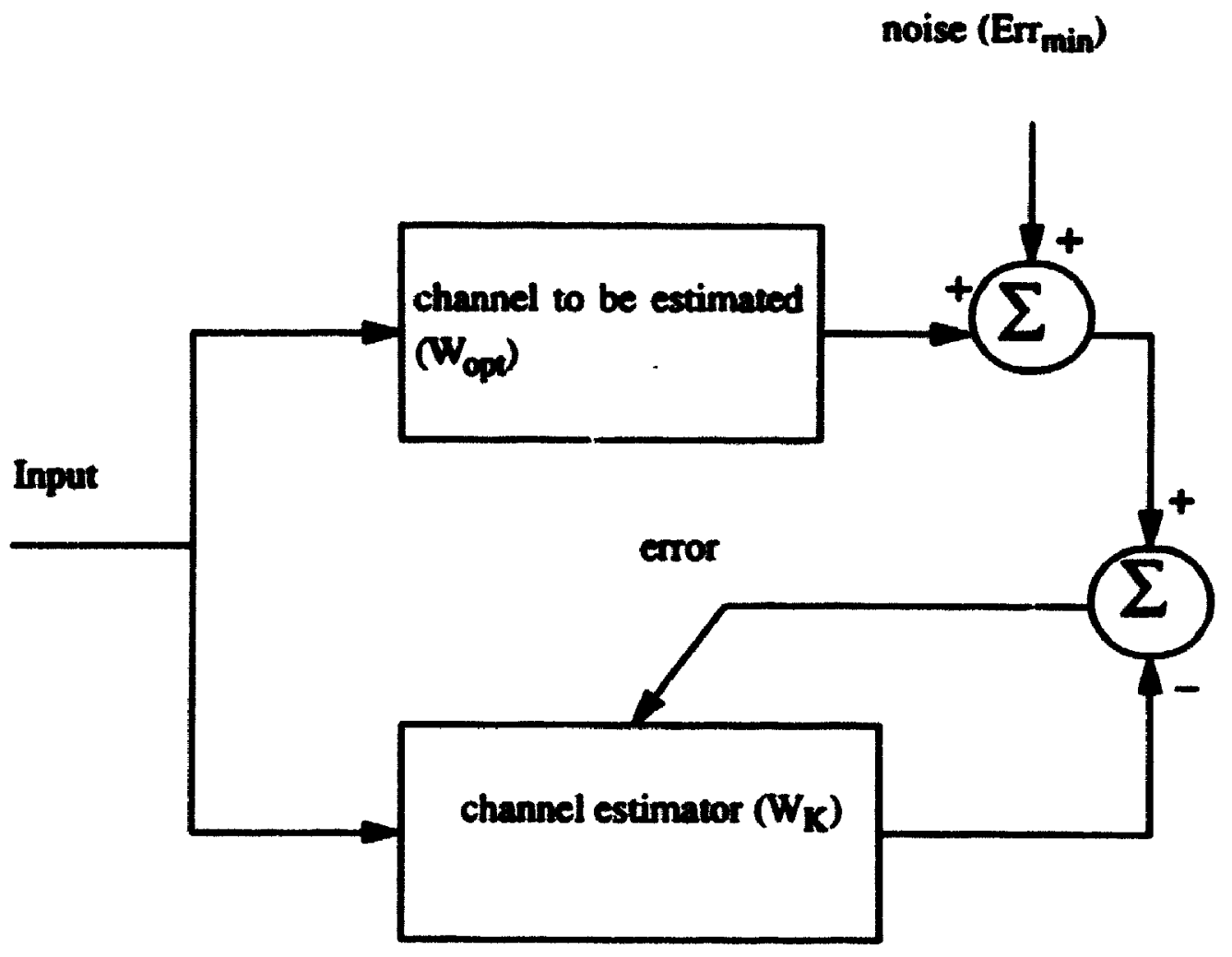

Figure (4.1) Set up for channel extimation 
noise variance: .01

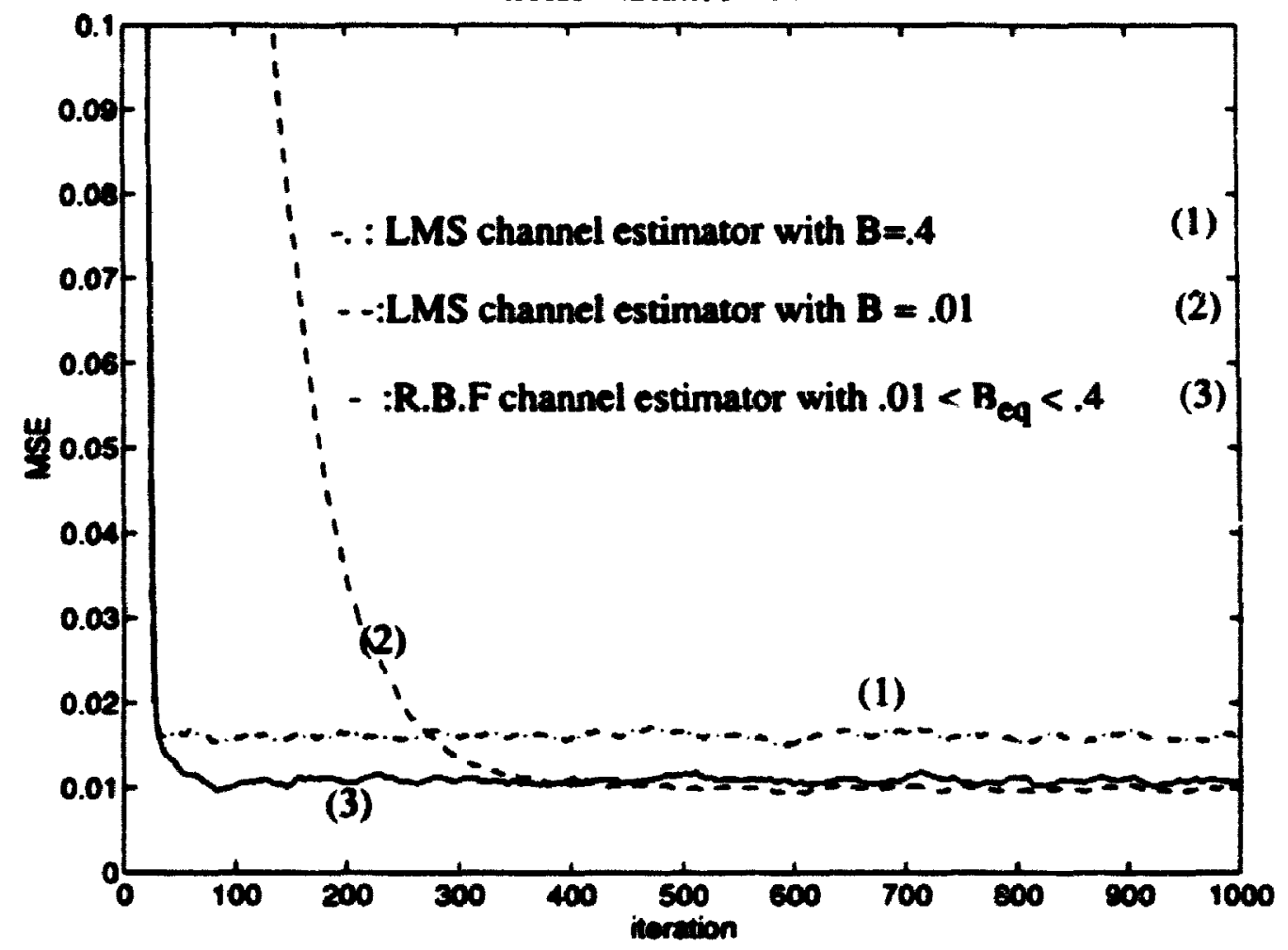

Figure(4.2) Chaned extimator at zero Doppler freq. and noise variance:.01 averaged over 100 independent channel 


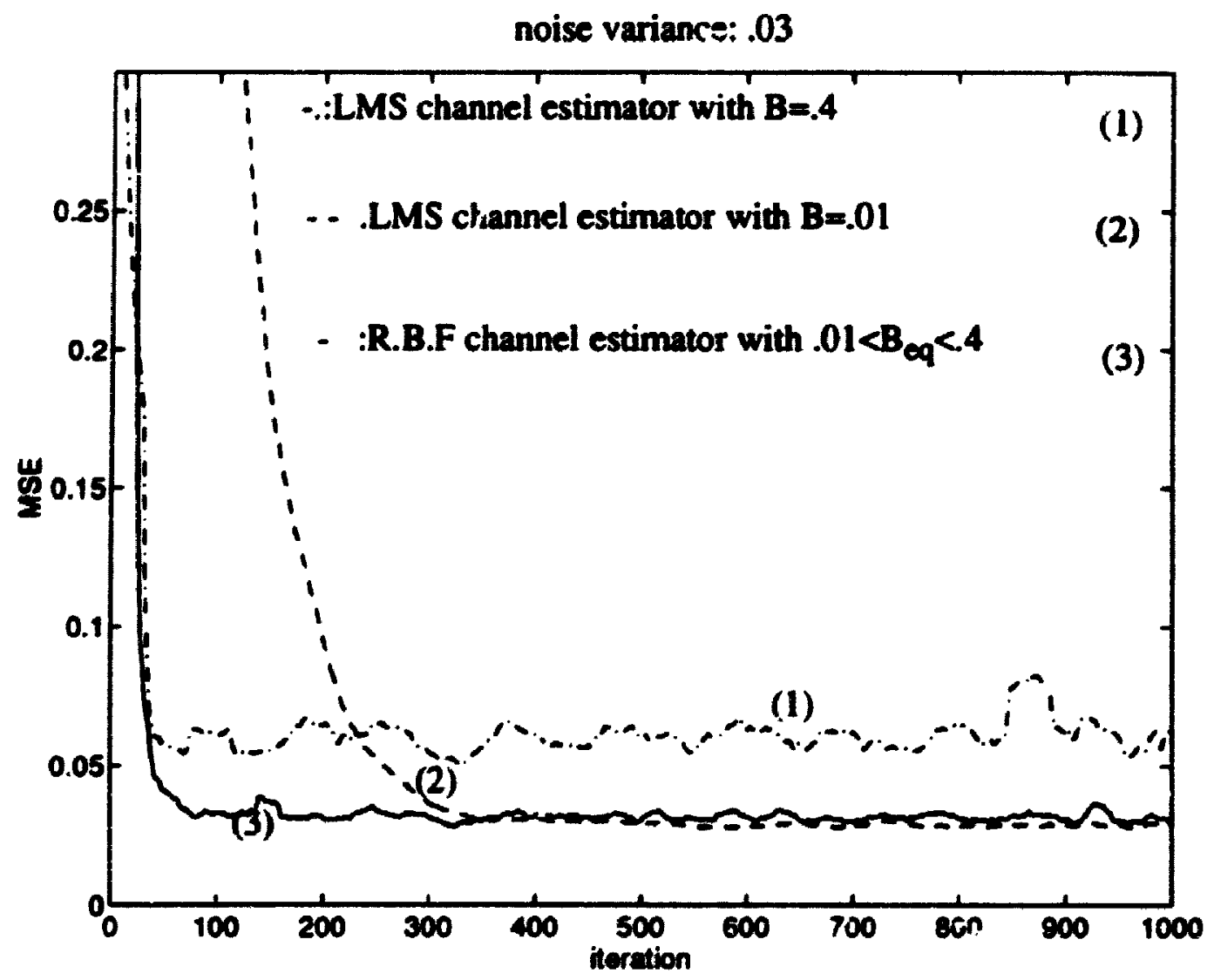

Figure (4.3) Channel eatimator at zero Doppler freq. and noise variance:.03 averaged over 100 independeat channel 
noise variance: .01

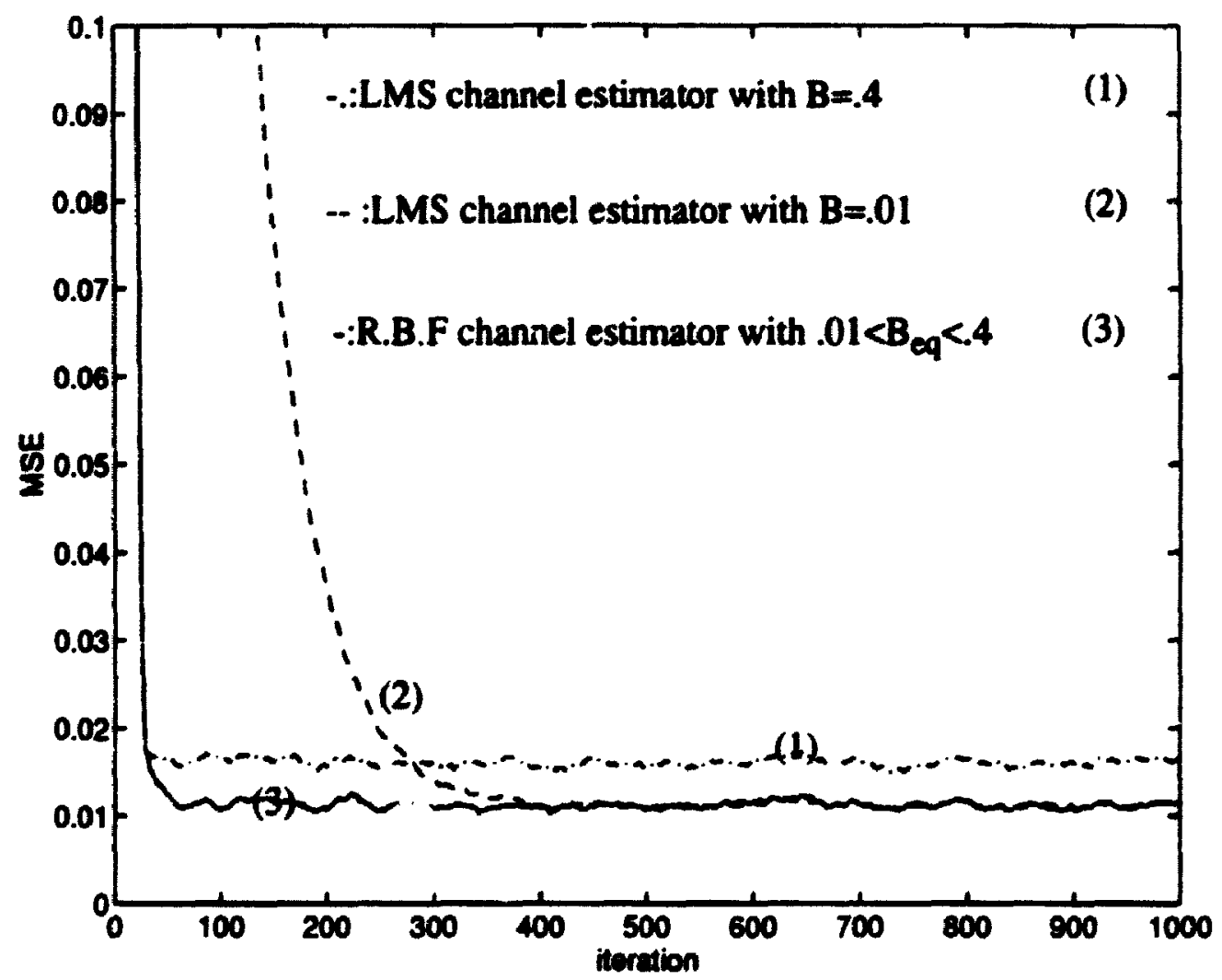

Figure (4.4) Channel extimator at Doppler freq: $2 \mathrm{~Hz}$

averaged over 100 independent channel 


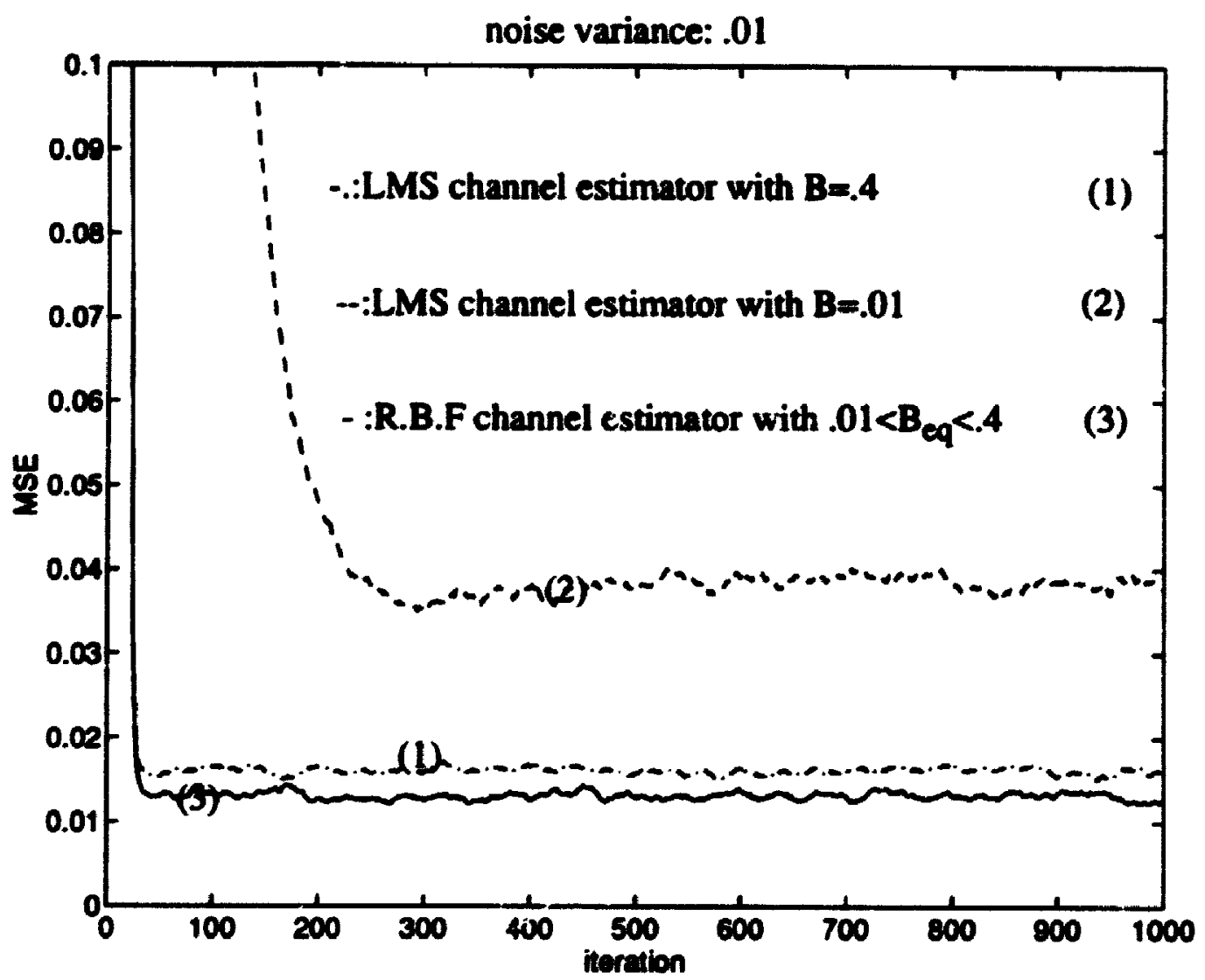

Fizure (4.5) Channel estimator at Doppler freq: $10 \mathrm{~Hz}$

averaged over 100 independent channel 


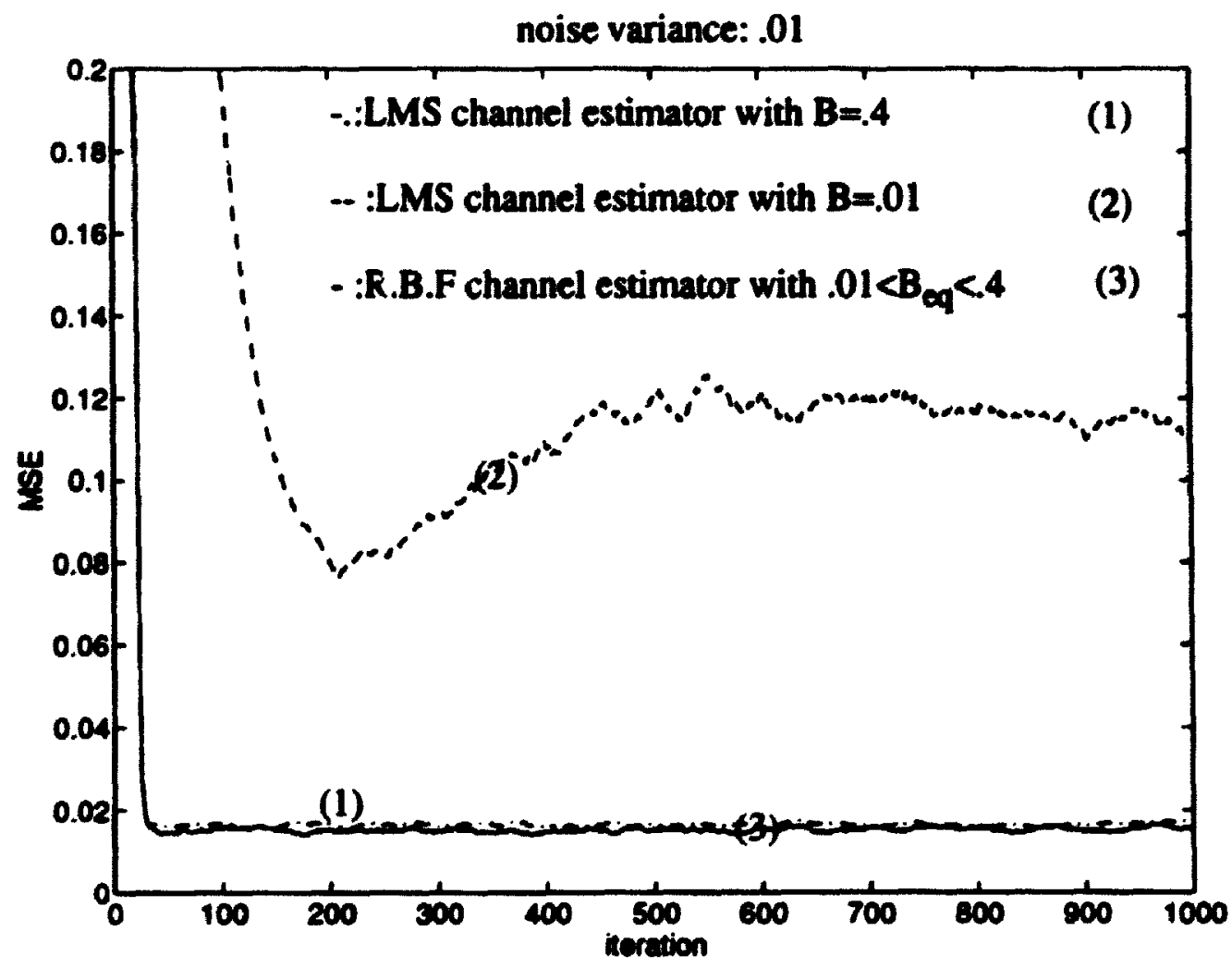

Figure (4.6) Channel extimator at Doppler freq:20Hz

averaged over 100 independent channel 


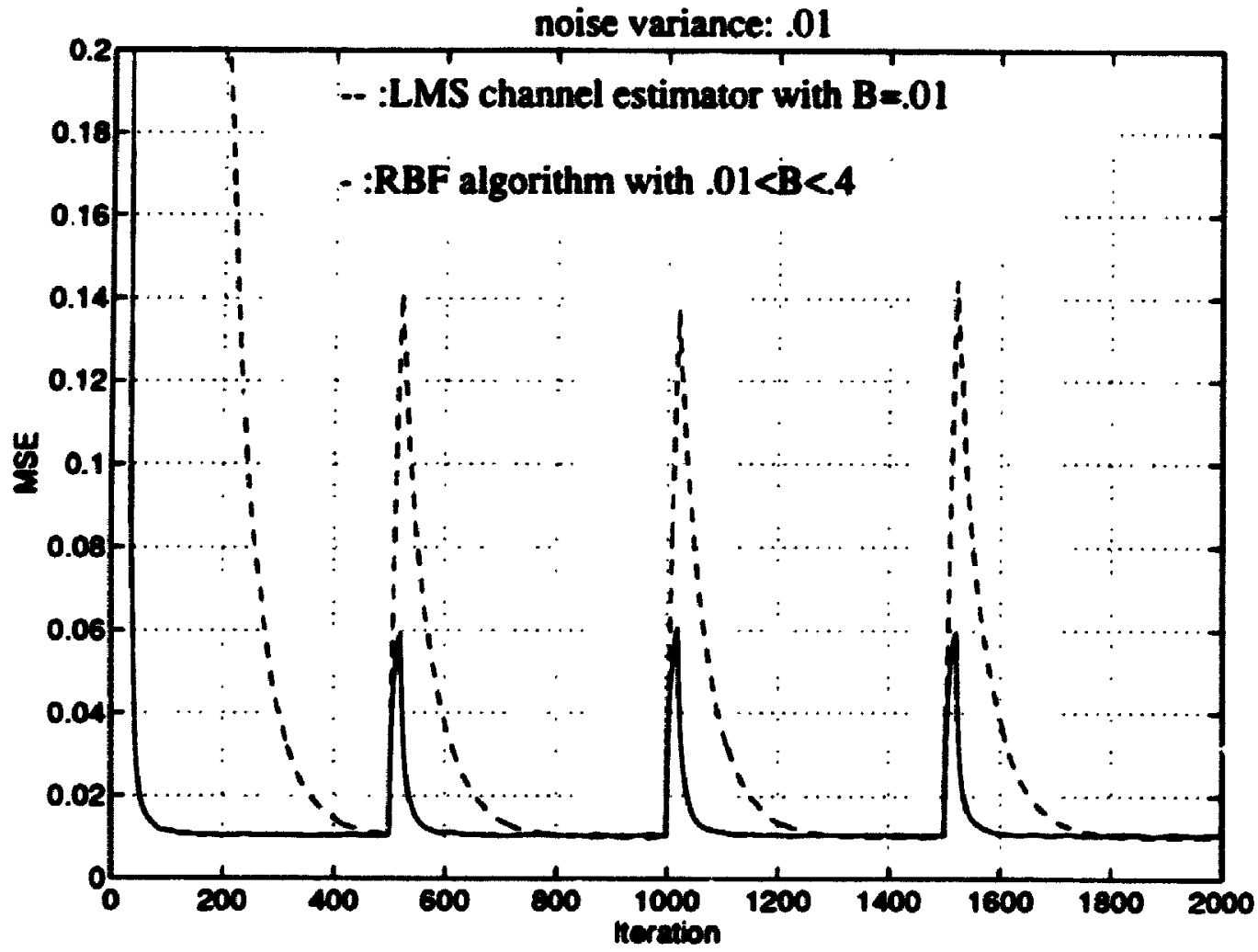

Figure (4.7) comparing LMS and R.B.F channel eatimator 


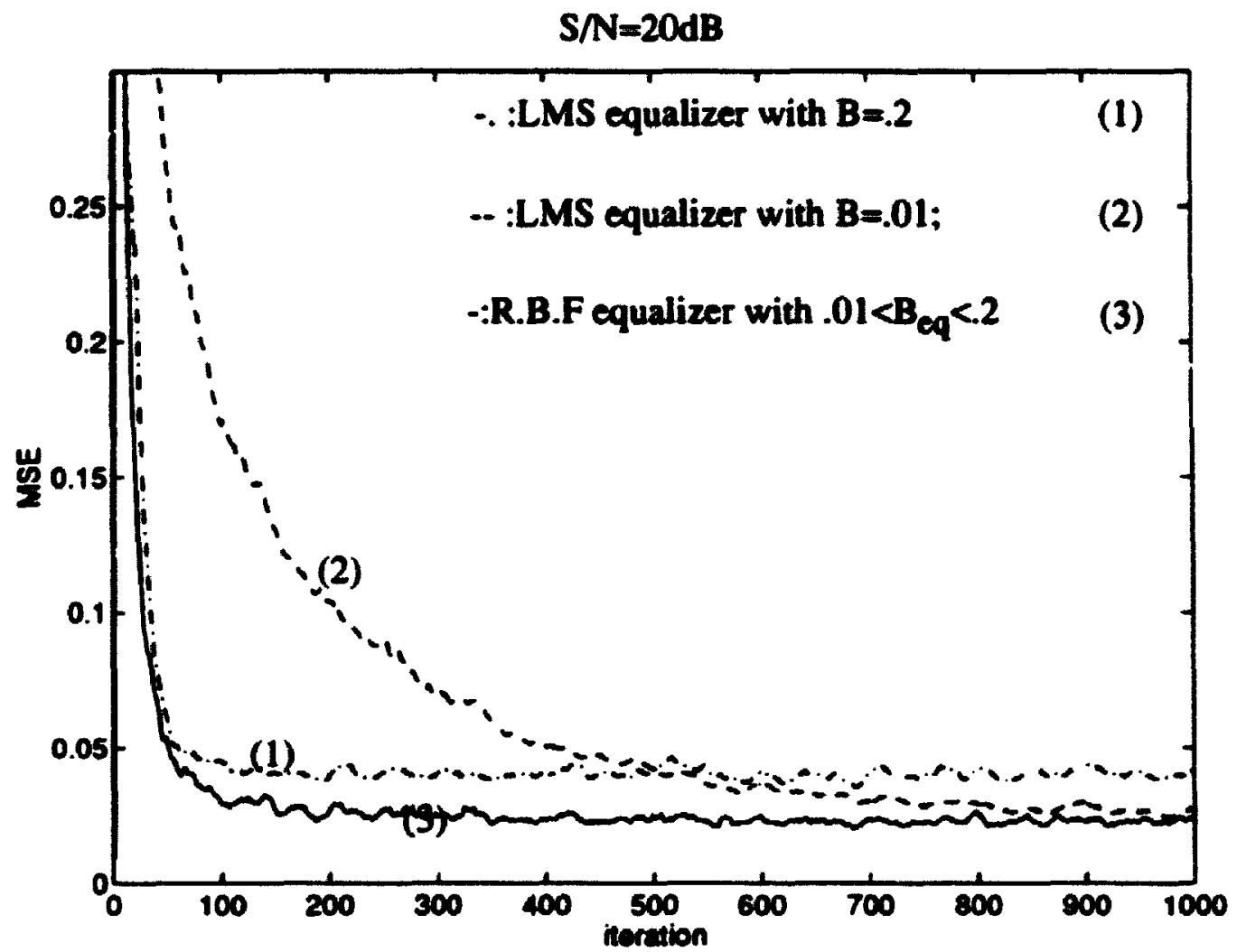

Figure (48) Equalizer performance at zero Doppler freq. and S/N=20dB averaged over 100 independent channel 


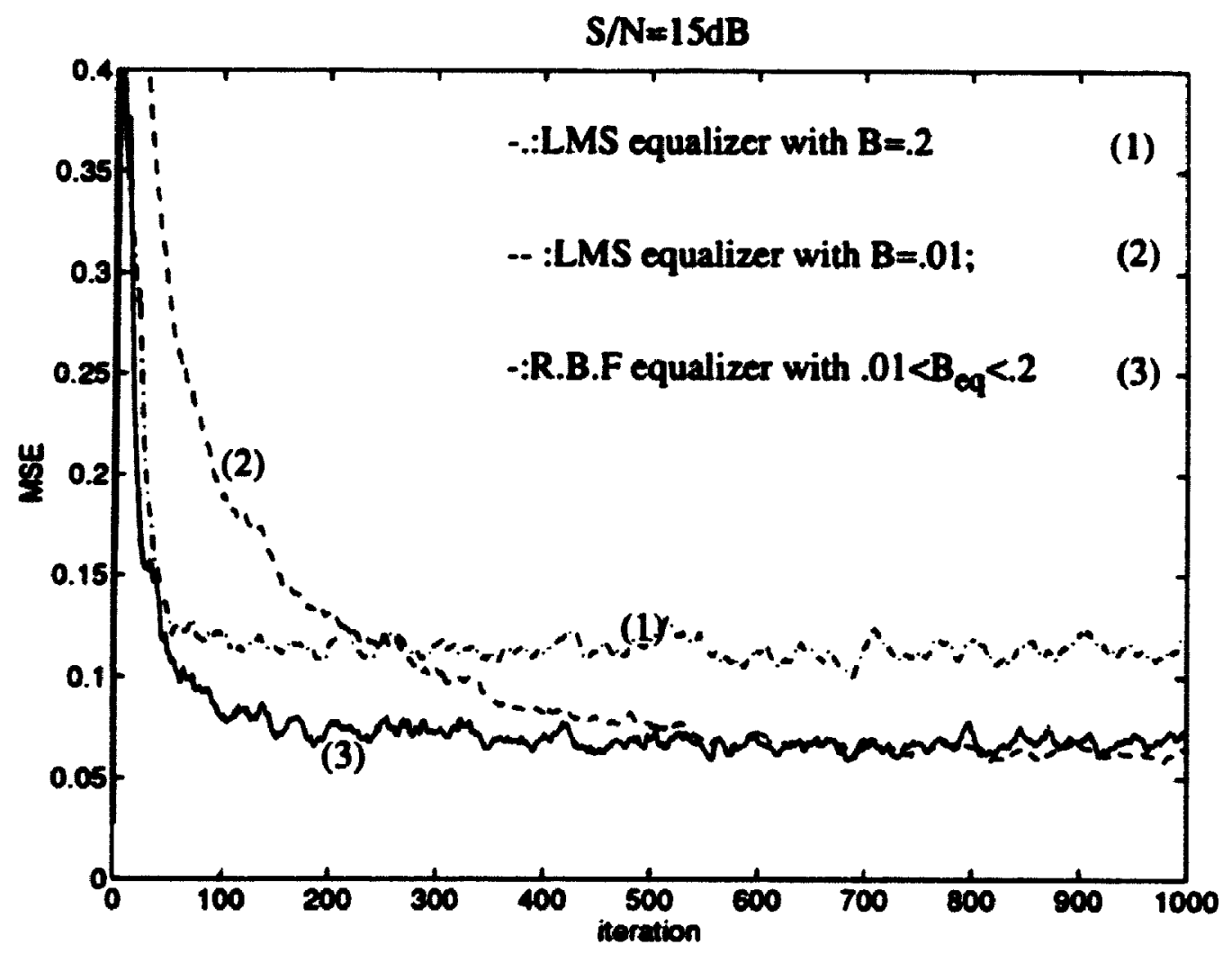

Figure (4.9) Equalizer performance at zero Doppler freq. and S/N=15dB averaged over 100 independent channel 


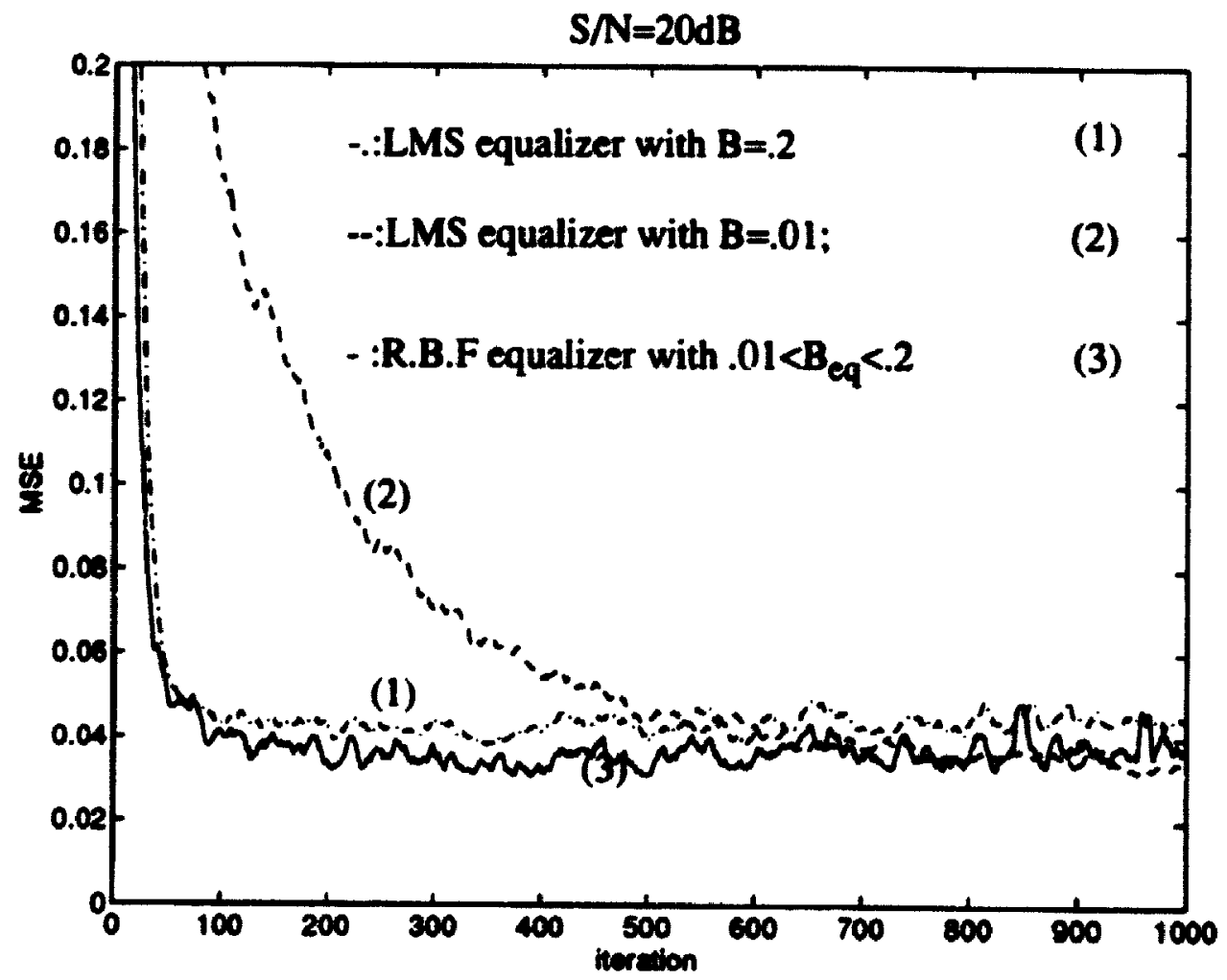

Figure (4.10) Equalizer performance at doppler freq. $=2 \mathrm{~Hz}$ averaged over 100 independent channel 


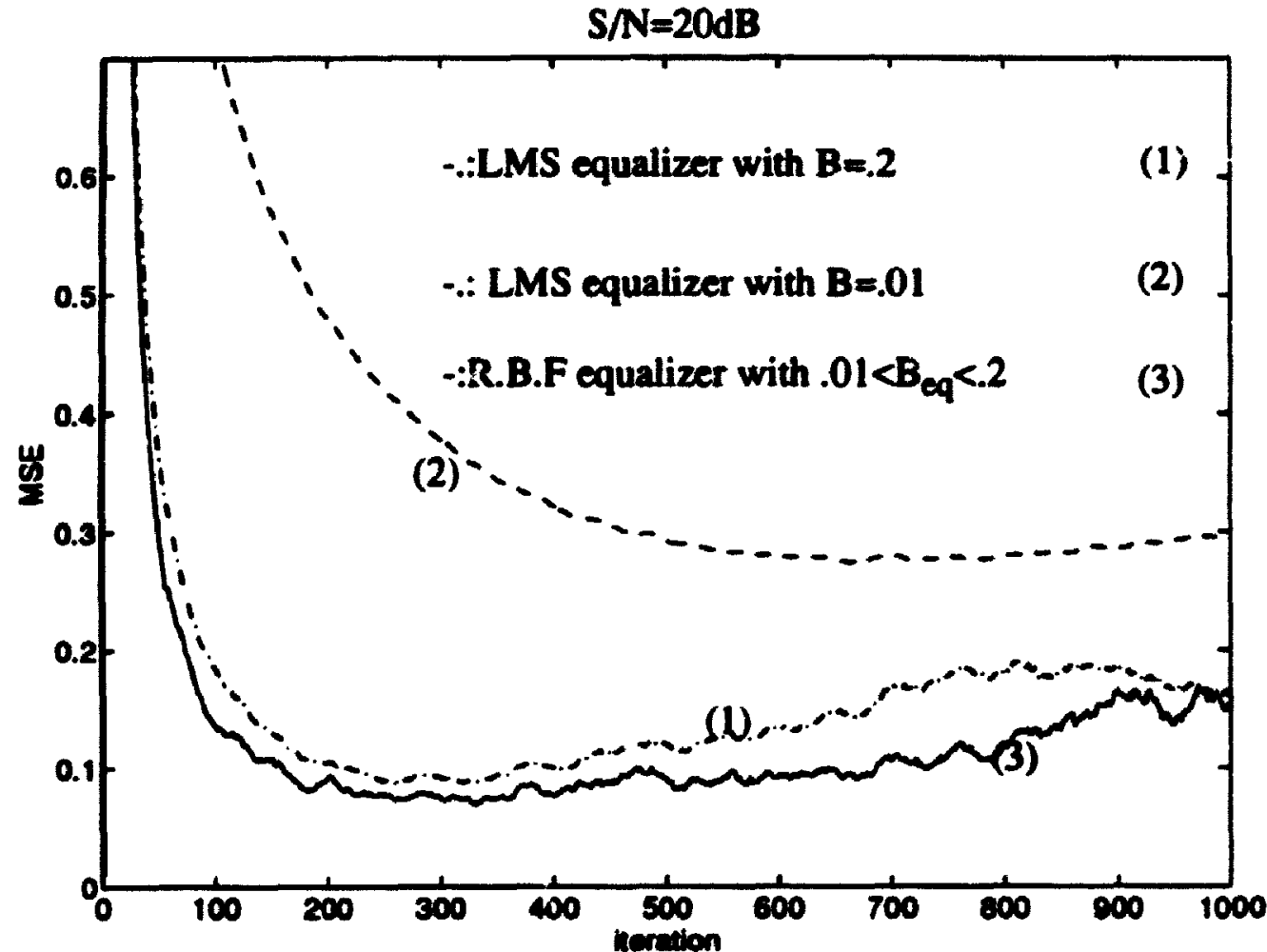

Figure (4.11) Equaliber performance at doppler freq $=10 \mathrm{~Hz}$

Doppler frequency:10 Hz (averaged over 100 independent channel) 


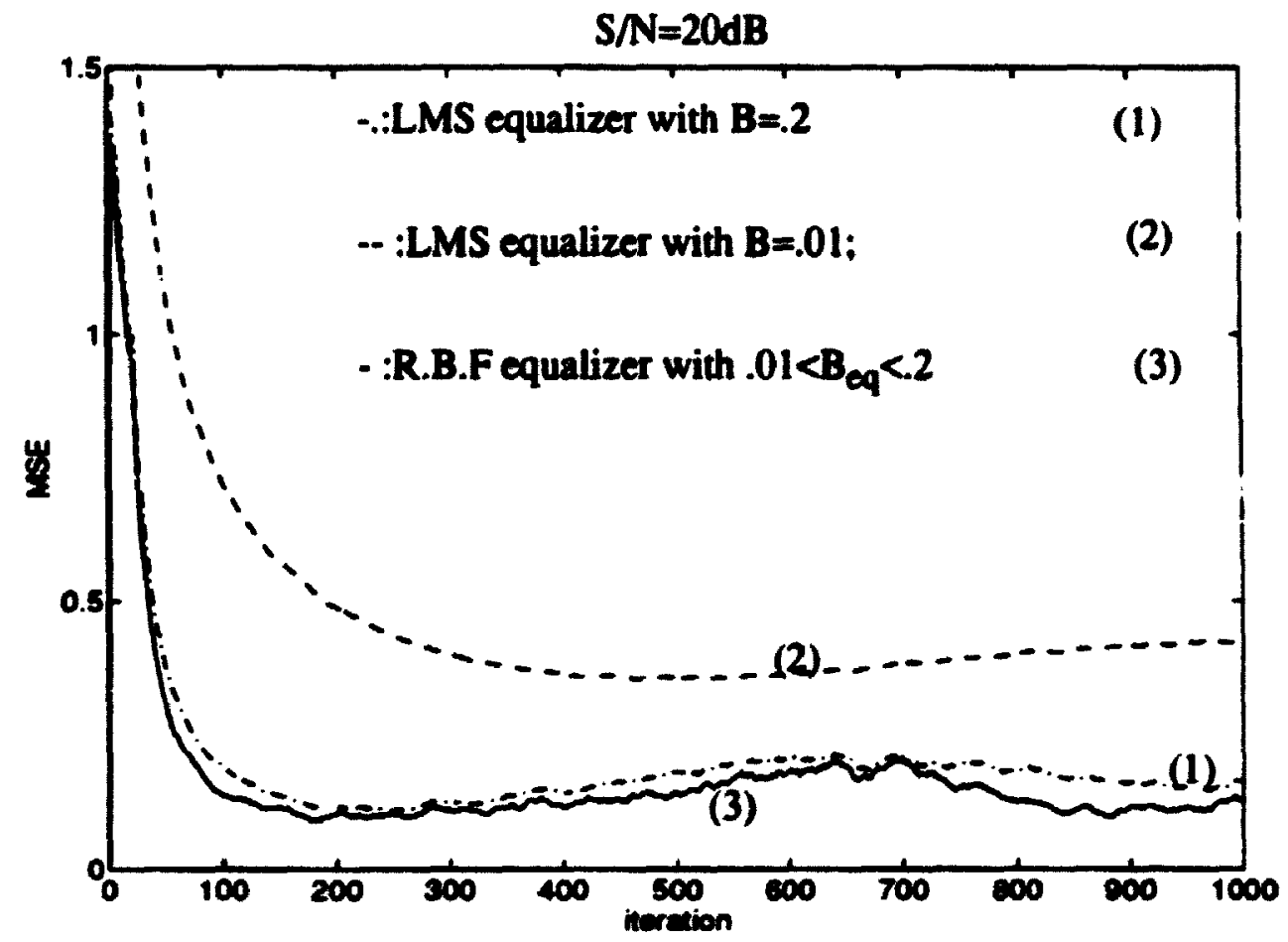

Figure (4.12) Equalizer performance at doppler freq. $=15 \mathrm{~Hz}$ averaged over 100 independent channel 


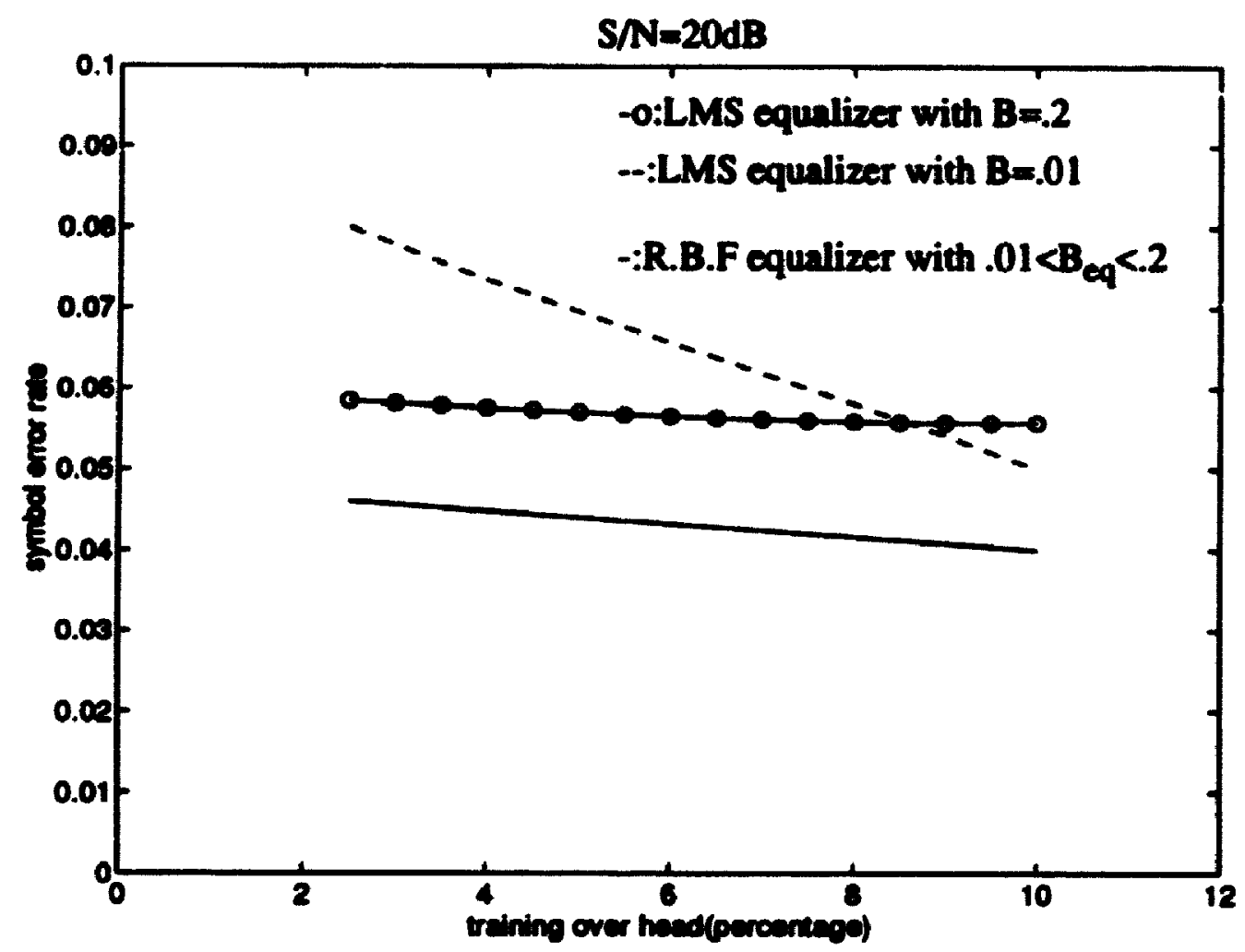

Pigure (4.13) Training overbead

two equal power chanael; SN equal to $20 \mathrm{~dB}$; frame leagth =1000symbols

Doppler frequency equal to SHz(avernged over 100 independent channel) 


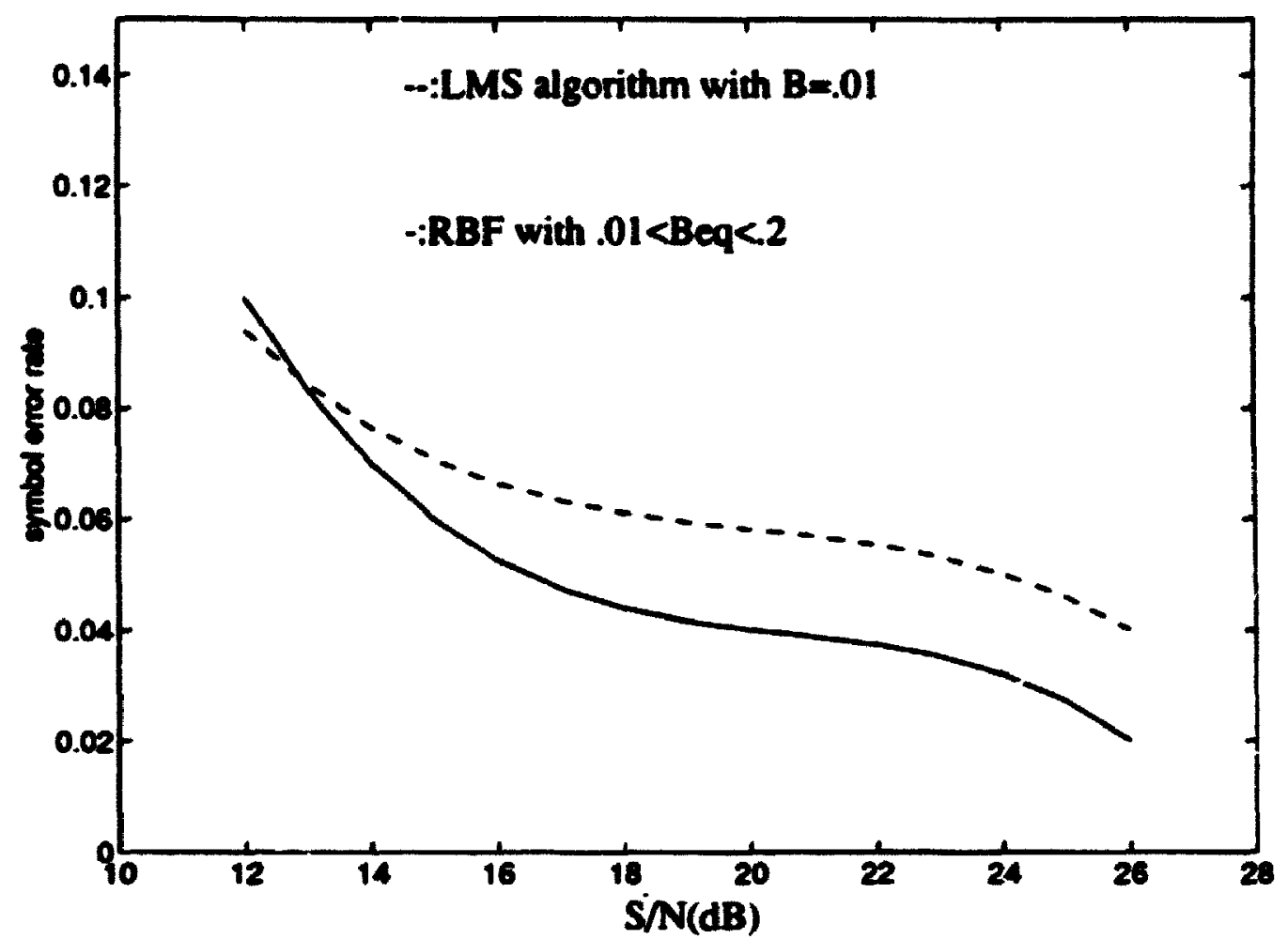

Figure (4.14) Comparing LMS and RBF Equalizx.:

Frame:1000 symbols with 100 symbols as over head.

Channel :two equal power ray channel.

Doppler frequency:0 Hax averaged over 100 independent channel) 


\subsection{BLOCK RADIAL BASIS FUNCTION EQUALIZER:}

\subsubsection{INTRODUCTION:}

The block R.B.F (B.R.B.F) equalizer has the same structure as the simple R.B.F equalizer discussed before. The cost function of B.R.B.F has several Gaussian terms, one for each cluster of vectors in the decision space.

In this section the updating equation for this equalizer is derived. The analysis shows that the B.R.B.F equalizer has faster convergence rate and better tracking performance than the simple R.B.F. The simulation results demonstrate the equalizer's performance and compare it with recursive least square (RLS) equalizer and LMS equalizer in a mobile channel scenario. Our investigation about the B.R.B.F equalizer is concluded by a computational romplexity discussion.

\subsubsection{BLOCK R.B.F ALGORITHM:}

The cost function in B.R.B.F algorithm is given by:

$$
T=\sum_{i=1}^{L+1} \exp \left(\left|y d_{i}-W_{k} \cdot x_{k-i+1}\right|^{2 / 2 \sigma^{2}}\right)
$$

where $\mathbf{L}$ is the block length (number of data in a block), $y \mathrm{~d}_{\mathbf{i}}$ is the ith desired data in block, $W_{k}$ is equalizer weight vector at time instant $k$ and $x_{i}$ is the input vector at instant i.

The update equation based on gradient descent algorithm is:

$$
\begin{aligned}
& W_{k+1}=W_{k}+\beta /\left(2 \sigma^{2}\right) \sum_{i=1}^{L+1} \exp \left(\left|y d_{i}-W_{k} \cdot x_{k-i+1}\right|^{2 / 2 \sigma^{2}}\right) \cdot x_{k-i+1}^{*} \cdot \underset{(4.31)}{\operatorname{er}} \\
& e r_{i}=\left(y d_{i}-w_{k} \cdot x_{k-i+1}\right)
\end{aligned}
$$

The B.R.B.F algorithm is explained with the aid of a flowchant in the next page: 


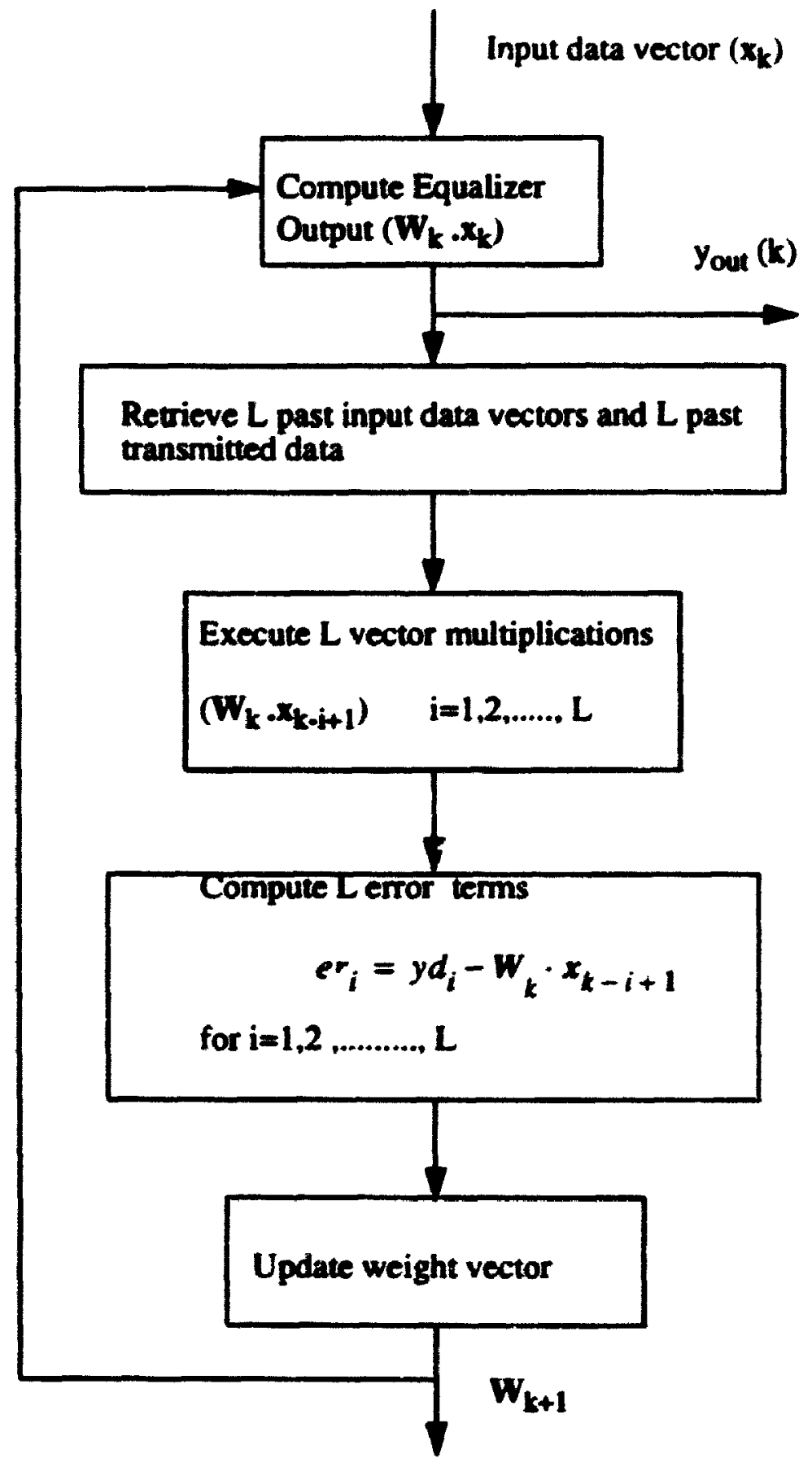

\section{B.R.B.F algorithm}




\subsubsection{ANALYSIS:}

In this section we explain and justify the extension of the R.B.F algorithm into the B.R.B.F.

The B.R.B.F algorithm can be considered as a modified Row Action Projection (R.A.P) algorithm [9][10]. The R.A.P algorithm first was developed as a method for solving system of linear equations[9] and later was applied for equalizer weight adjustment[10]. In this subsection, first we explain R.A.P algorithm briefly and after that the relation between the R.A.P algorithm and the B.R.B.F is discussed.

The problem of finding equalizer coefficients can be formulated as a system of linear equations:

$$
\begin{aligned}
& {\left[\begin{array}{c}
y d_{k} \\
y d_{k-1} \\
\vdots \\
y d_{k-L+1}
\end{array}\right]=\left[\begin{array}{c|c}
r_{k} & y d_{k-1} \\
r_{k-1} & y d_{k-2} \\
\vdots & \vdots \\
r_{k-L+1} & y d_{k-L}
\end{array}\right] \times\left[\begin{array}{c}
a_{k} \\
b_{k}
\end{array}\right]+\left[\begin{array}{c}
e_{k} \\
e_{k-1} \\
\vdots \\
e_{k-L+1}
\end{array}\right]} \\
& r_{k}=\left[\begin{array}{lllll}
r_{k-N+1}, r_{k-N+2}, & \cdots, r_{k-1} & , r_{k}
\end{array}\right] \\
& y d_{k-1}=\left[y d_{k-1}, y d_{k-2}, \quad . \quad, y d_{k-M+1}, y d_{k-M}\right]
\end{aligned}
$$

In the above system of equations the 'rector $\mathbf{r}_{\mathbf{k}}$ represents input vector at time $k$, vector $y d_{k-1}$ is the decision vector which represents the past $M$ feedback values at time $k$. vectors $a_{k}$ and $b_{k}$ are the coefficients of the feedforward and feedback filters respectively.

At the $\mathrm{k}^{\text {th }}$ instant the above linear equations can be written in matrix form as:

$$
y d_{k, L}=x_{k, L} \times w_{k}+e_{k, L}
$$


$L$ is the number of rows in the time varying matrix $X_{k, \downarrow}$. The rows of this matrix are input vector $\mathbf{x}_{\mathbf{j}}$.

The LMS algorithm uses the update equation:

$$
W_{k+1}=W_{k}+\beta\left[y d_{k}-W_{k} x_{k}\right] x_{k}^{*}
$$

to find the optimum weight vector at each time instant $k$ by using only one row of the data matrix $\mathbf{X}_{\mathbf{k},\rfloor}$ for each update. In the R.A.P algorithm the incorporation of multiple data as in the RLS algorithm with the use of gradient descent algorithm found in the LMS algorithm are combined. The R.A.P algorithm solves the equation (4.32) (to find optimum weight vector) by an iterative method as follows:

$$
\begin{aligned}
& W_{k, i+1}=W_{k, i}+\beta \cdot e r_{i}\left(x_{k-i+1}\right)^{*} \\
& e r_{i}=y d_{i}-W_{k, i} x_{k-i+1} \\
& i=1,2, \cdot \cdot l+1 \\
& k=t i m e-i n d e x
\end{aligned}
$$

where er $r_{i}$ is the erro: at the ith equation and $\beta$ is the step size.

In the above algorithm for a block of data with the length of $L$. the oldest equation related to the oldest input vector is updated based on gradient descent algorithm. The updated vector coefficient is used to compute the next error value and to update the next equation. This process continues until $L$ equations have been updated, $W_{k \perp+1}$ is the updated set of coefficients for equalizer $\left(\mathbf{W}_{k+1}\right)$. This algorithm has a performance similar to the RLS equalizer, with less complexity [10].

A geometrical interpretation of the R.A.P method can be presented as follow:

The R.A.P algorithm performs a sequence of projections of coefficient vector toward the hyperplanes orthogonal to the rows of the data matrix $X_{k J}$. The distance of the hyperplane from the origin is $y d_{j}$. This geometrical interpretation of the operation is shown in figure 4.15. 


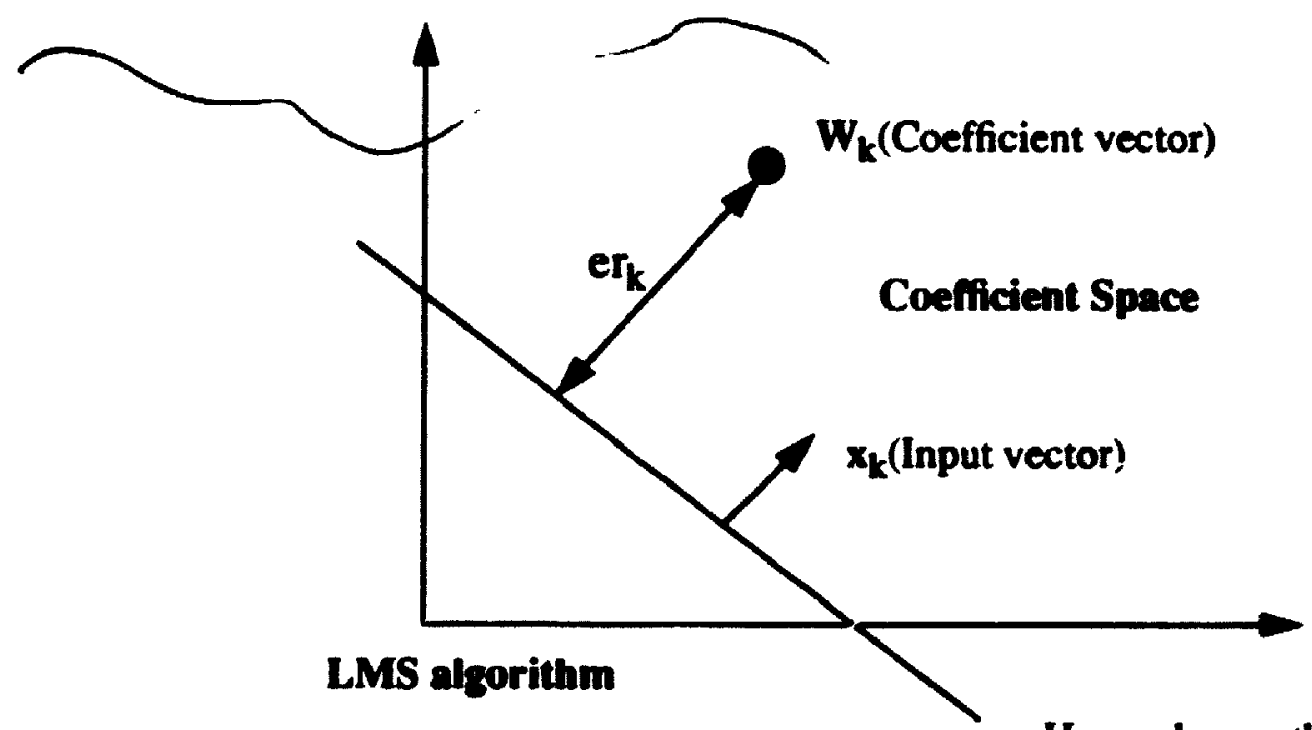

Hyperplane orthogonal to the data vector

$$
W_{k+1}=W_{k}+\beta \cdot e r_{k} \cdot x_{k}
$$

$x_{k}$

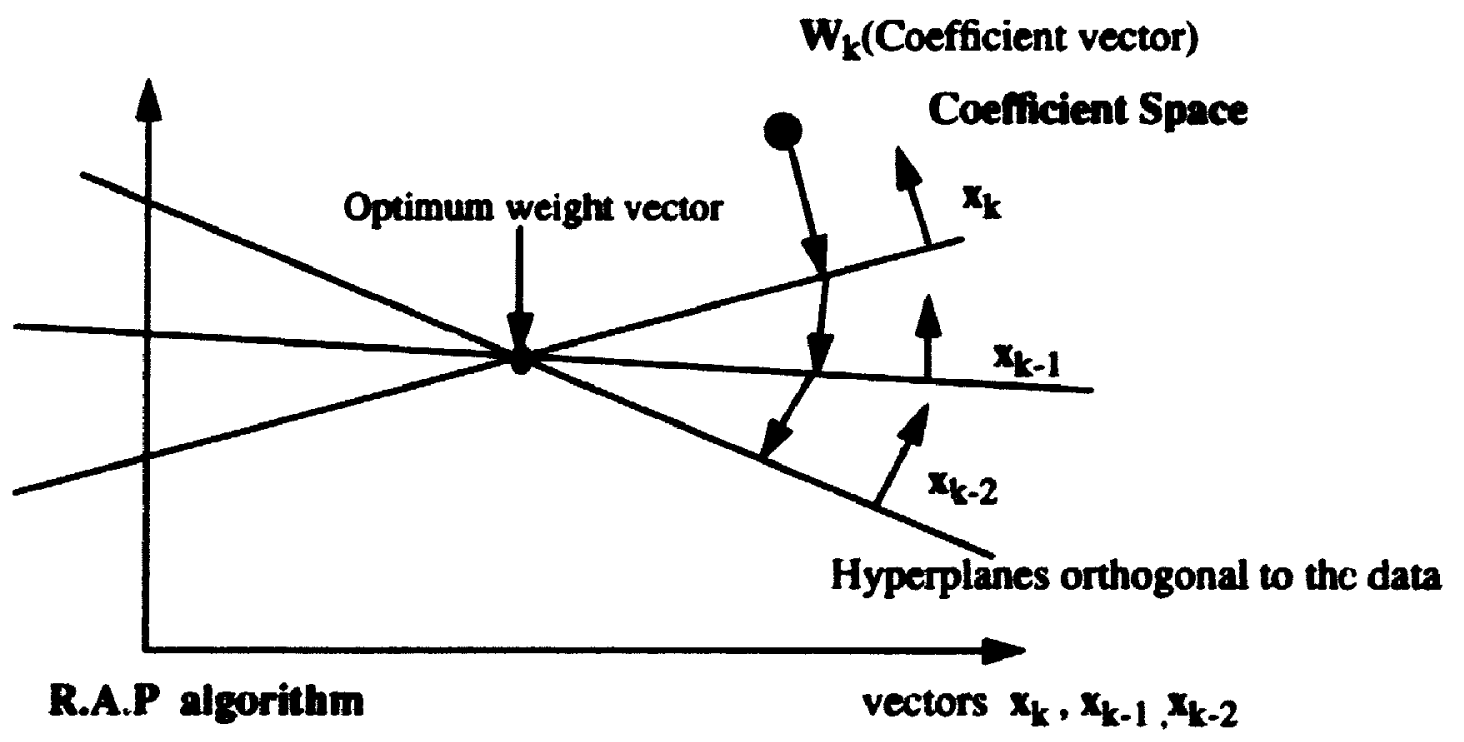

Figure (4.15): Geometrical interpretation of R.A.P algorithm 
The difference between the R.A.P algorithm and the RLS algorithm is in the way that they compute the inverse matrix to solve eq. (4.35). The RLS algorithm solves eq (4.35) as [38]:

$$
W_{R L S}=\left[X^{T} \cdot X\right]^{-1} X^{T} y d_{k l}
$$

A more general form of the least-squares solution of eq. (4.35) is

$$
w_{P I}=x \dagger \cdot y d_{k l}
$$

where $X^{\dagger}$ is the Moore-Penrose pseudo-inverse of the data matrix X [45]. The pseudoinverse solution is unique and is the least - squares solution of the minimum norm [9]. In [9] it has been shown that the R.A.P algorithm converges to $\mathbf{W}_{\mathbf{P I}}$.

There are some advantages in using the pseudo-inverse solution as follow:

(I): The pseudo-inverse solution is identical to the least squares solution only if the data matrix is full rank. When $X$ is rank deficient the inverse matrix in eq. (4.39) does not exist. The pseudo-inverse, however is well defined for any matrix regardless of its rank [9].

(II): The eigenvalue spread in RLS solution is the square of that in pseudo inverse solution [9] . Thus, the RAP algorithm will operate on a system that is better conditioned than the RLS system.

(III): In [9] it has been shown that the R.A.P algorithm is a regularized algorithm. A regularized inversion algorithm applies a taper to the inversion of the singuler values of data matrix, such that the modes with low signal to noise ratios are attenuatid prior to the inversion. This mechanism prevents noise enhancement existing in the RLS algorithm.

The B.R.B.F algorithm, like R.A.P, is a multiple projection algorithm. Their differences are as follow:

\section{(I): Variable adaptation rate:}

In the B.R.B.F, adaptation rate coefficient in each projection in eq. (4.38) is replaced by a Gaussian function of square error (R.B.F algorithm). This modification gives a better adjustment and faster convergence at each projection as it has been shown before. 


\section{(II): Fixed weight vector in the data block:}

In the B.R.B.F. $W_{k, i}$ is replaced by $W_{k, 1}$ (the first coefficient vector in the data block) in computing $\mathrm{er}_{\mathrm{i}}$. This modification is justified when the block length is short. The reason is, in a short block of data, equalizer coefficients don't change considerably. This modification makes it possible to compute error values in a block of data at the same time and in parallel rather than the serial method as in the R.A.P algorithm.

By applying these modifications on eq(4.38) we derive the following equations:

$$
\begin{aligned}
& W_{k, i+1}=W_{k, i}+\beta\left(e r_{i}\right) \cdot e r_{i} x_{k-i+1}^{*} \\
& \beta\left(e r_{i}\right)=\beta /\left(2 \sigma^{2}\right) \cdot \exp \left(\left|e r_{i}\right|^{2} /\left(2 \sigma^{2}\right)\right) \\
& e r_{i}=y d_{i}-W_{k, 1} x_{k-i+1} \\
& i=1,2, \cdot l+1 \\
& k=t i m e-i n d e x
\end{aligned}
$$

By computing $W_{k \perp+1}$ in term of $W_{k, L}$ we derive the following updaie equation:

$$
w_{k+1}=w_{k}+\sum_{i=0}^{L+1} \beta\left(e r_{i}\right) e r_{i}\left(x_{k-i+1}\right)^{*}
$$

which is the B.R.B.F update algorithm.

The discussion in this sub-section illustrated the fundamental difference among the B.R.B.F, LMS and R.B.F algorithms. All these algorithms are projection update methods. However, the B.R.B.F uses multiple projections for every coefficient update with multiple variable adaptation rates, while LMS and R.B.F algorithms use only one projection per update. 


\subsubsection{SIMULATION RESULTS:}

Series of simulation experiments were conducted to investigate various aspects of the B.R.B.F equalizer and to compare it's performance with the RLS and LMS equalizers. During the simulation, for the B.R.B.F algorithm minimum value of the equivalent adaptation rate is .01 and maximum value of adaptation rate is set equal to .2 (for each Gaussian term in the algorithm). For the LMS equalizer; adaptation rate is chosen based on series of short term simulations and optimum value is selected at each case. For the RLS equalizer forgetting factor is set to .9 and direct form of the RLS equalizer is used.

Figure 4.16 presents the effect of variance parameter on the convergence speed of the B.R.B.F equalizer. It can be seen that for a relatively wide range of values for this parameter, the equalizer has the same performance.

Figure 4.17 and 4.18 demonstrate the effect of block length on the convergence speed and tracking ability of the B.R.B.F equalizer in fading channel. Simulation results reveal that a four symbols duration block is a good compromise between performance and computational complexity. It is based on this observation that most of the simulation results presented in this section are for equalizer with block length equal to four.

In Figure 4.19 the convergence speed of the three different equalizers LMS, B.R.B.F and R.L.S equalizer in three ray channel are compared. It is clear that B.R.B.F equalizer with block length equal to 4 symbols has almost the same performance as R.L.S equalizer.

The next set of simulation is aimed to study the effect of additive Gaussian noise power on equalizer performance. Since it is known that RLS and LMS equalizers are quite sensitive to noise [39] and have noise amplification property, it is important to compare the effect of additive noise power on the RLS and LMS equalizer with the B.R.B.F equalizer. Figure 4.20 demonstrates the results of this simulation experiment at doppler frequency equal to $50 \mathrm{~Hz}$. As it can be seen, at low S/N the RLS performance is worse than B.R.B.F equalizer.The reason is due to noise enhancement property of RLS algorithm.

Figures 4.21 and 4.22 show the effect of rate of changes in the channel on the equalizer performance at an specific signal to noise ratio ( $\mathrm{S} / \mathrm{N}=20 \mathrm{~dB}$ ). Obviously R.B.F algorithm outperforms the LMS algorithm with a large margin. From this figure, it is clear that the 
RLS algorithm tracks variations inside the channel better than R.B.F algorithm but it was achieved with considerable complexity.

The performance of the B.R.B.F with two branch diversity is shown in fig 4.24. In this experiment two branch space diversity is employed and $S / N$ is set equal to $20 \mathrm{~dB}$. Receiver structure is composed of two fractionally spaced equalizer, one for each branch. One common decision device has been employed (see fig4.23). In this structure each equalizer is updated independently based on the difference between the equalizer output and decision device output. The equalizer output with the largest input power is selected as the current input to the decision device. This experiment shows that the R.B.F algorithm advantage over L.M.S equalizer holds even with having space diversity.

Error propagation is a major problem for any decision feed back equalizer in fast fading channel [37]. Fig 4.25 and 4.26 show the effect of error propagation on the B.R.B.F equalizer at different doppler frequencies and different $\mathbf{S} / \mathbf{N}$. Simulation results show that there is no advantage in using Block radial basis function instead of RLS equalizer. Since it is known that long frame will cause high error rate [8] in this experience the frame length is considered to be $\mathbf{3 0 0}$ symbols with $\mathbf{3 0}$ symbols as training data. Because of short training period; LMS equalizer is not considered in this part of the work.

\subsubsection{COMPUTATIONAL COMPLEXITY:}

From B.R.B.F update equation it is clear that the number of complex multiplications (as a measure for complexity) is $L$ times of that for simple R.B.F, where $L$ is the block length. Therefore, the total number of complex multiplication is $L(2 N+2)$ in B.R.B.F algorithm.

The computational complexity of the B.R.B.F equalizer with two different block lengths is compared with RLS, FRLS and LMS equalizers in figure 4.27. 


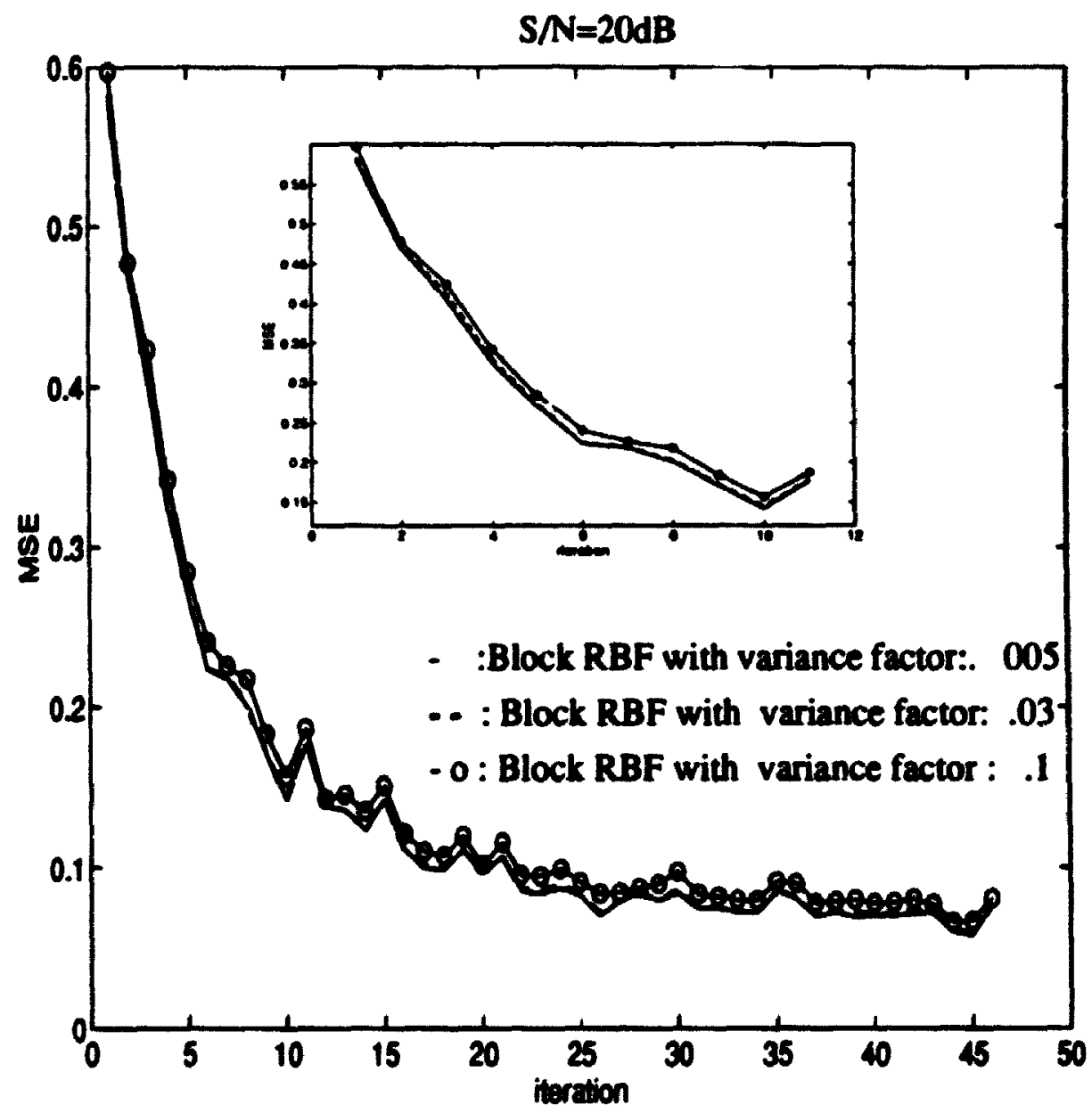

Figure (4.16) Convergence speed / variance parameter

channel : Tbree ray channel model at zero Doppler frequency (avereged over 500 independent channel;

Equalizer : fractionally spaced DFE(3 forward tapa,2 feedback taps). block length :4 symbols 


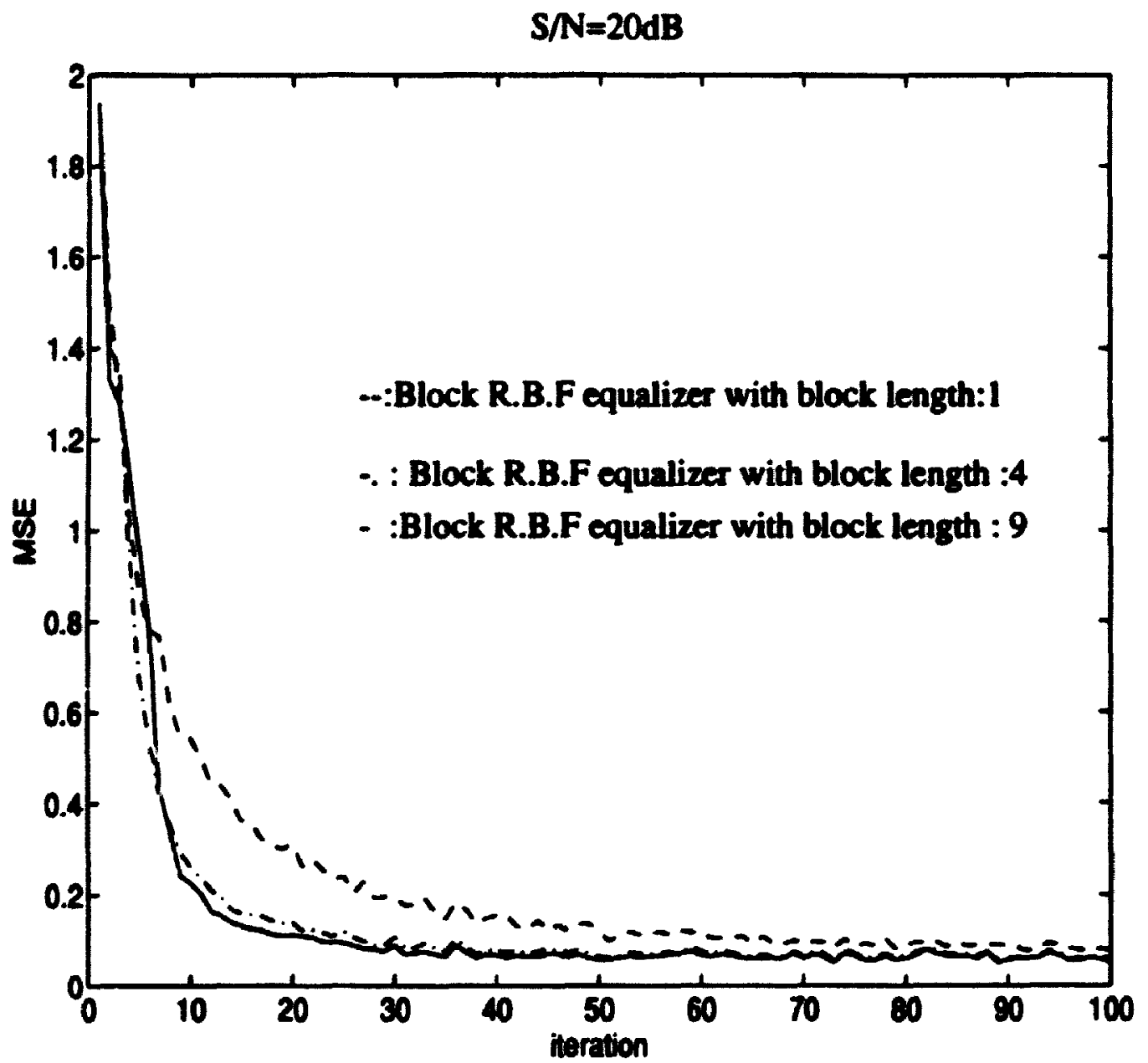

Figure (4.17) Convergence rate/Block length

channel : Three ray channel model at zero Doppler frequency averaged over 1000 independent channels

Equalizer : fractionally spaced DPE(3 forward tape ,2 feedback taps). 


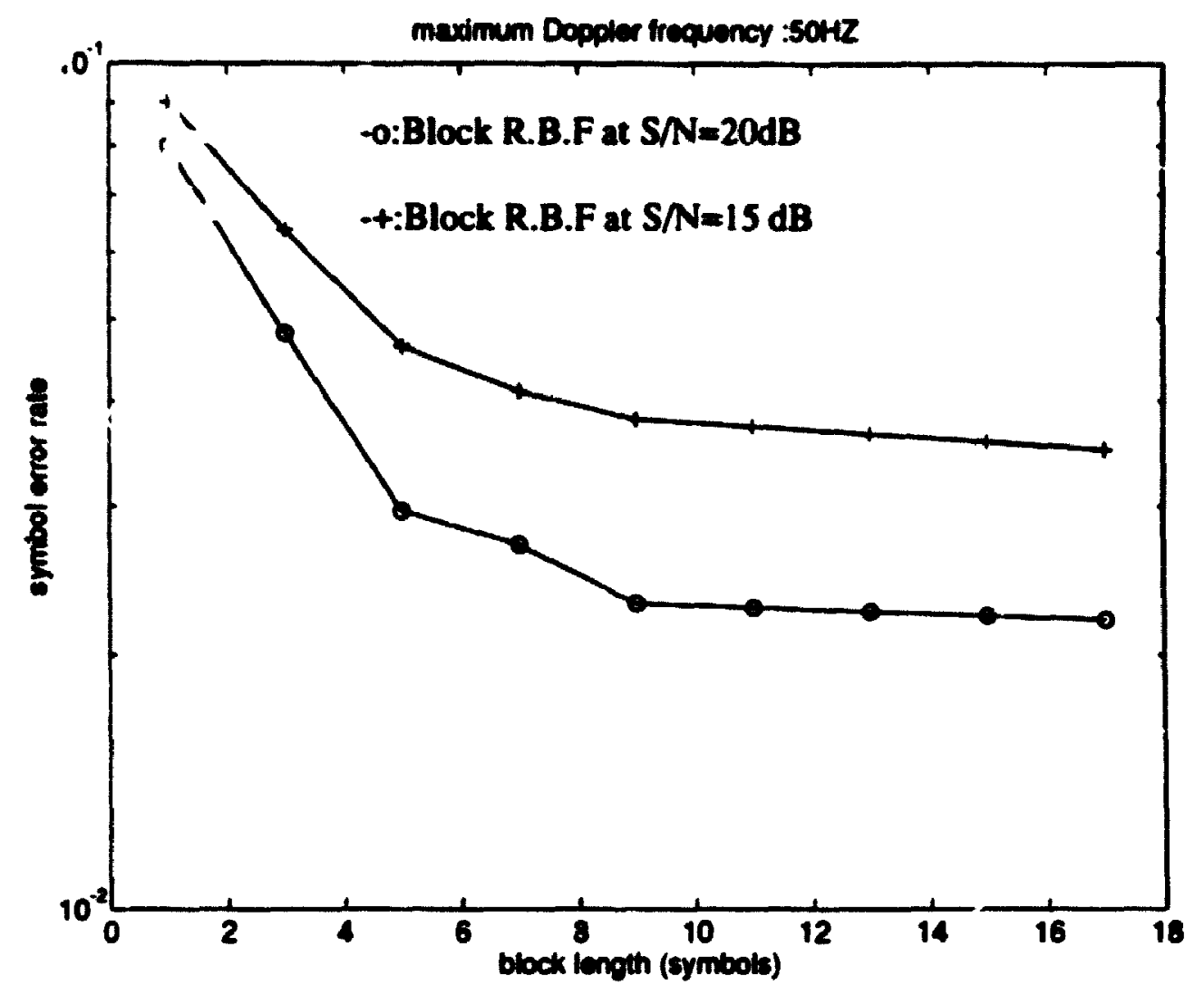

Figure (4.18) Symbol ratedBlock length

effect of block length in Block R.B.F equalizer performance at two different signal to noise ratio is invectipated

frame: SCO symbols with 50 symbols as training

channel: Three ray channel model at SOHz Doppler frequency

Equalizer: fractionally spaced DFE(3 forward taps,2 feedback taps). 


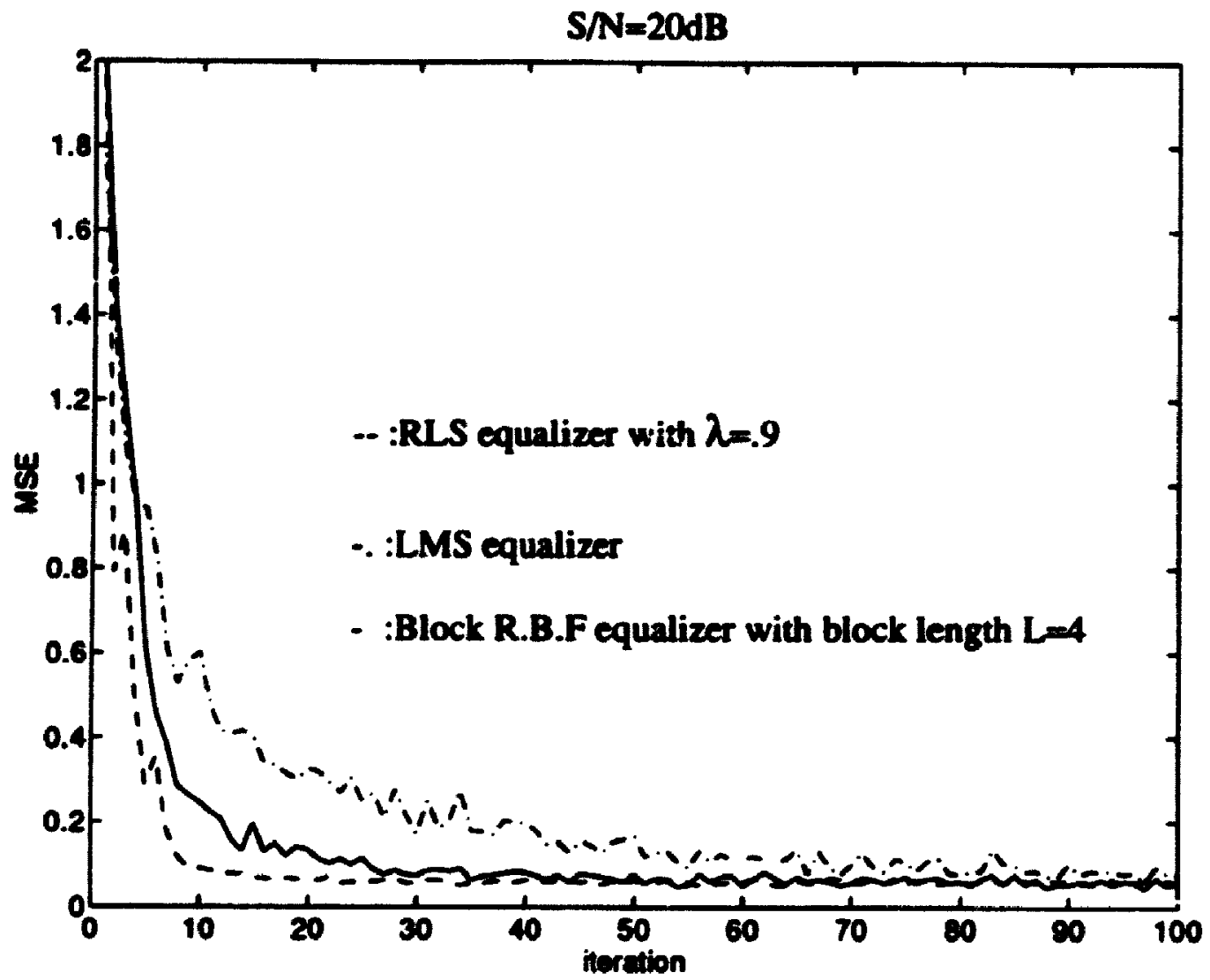

Pigure (4.19) Equalizers's Convercence rate

channel: Three ray channel model at zero Doppler frequency averaged over 500 independent channel

Equalizer: fractionally speced DFE(3 forward taps,2 feedbeck tapa). 


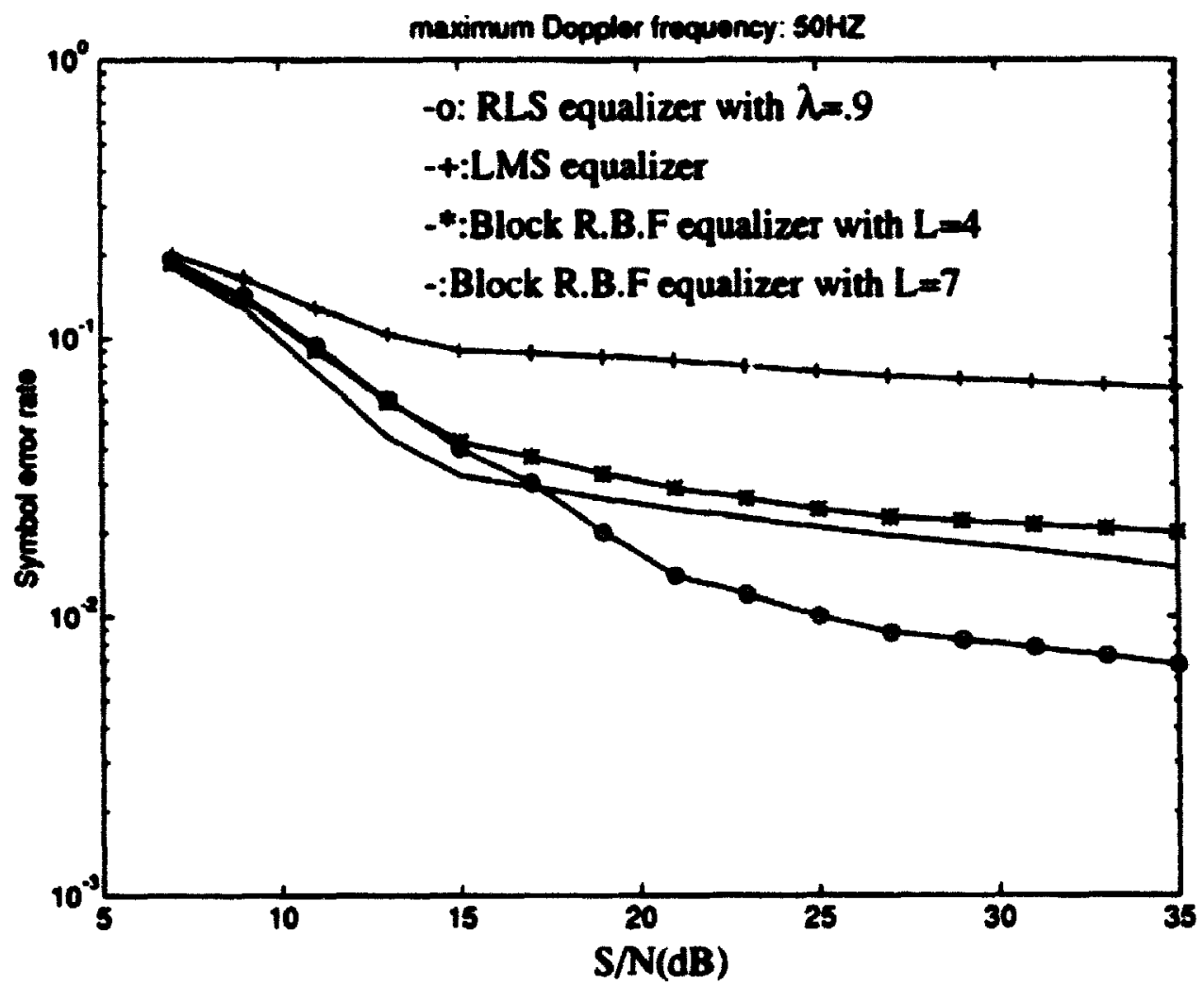

Figure (4.20) Comparing different equalizer performances

frame:500 symbols with 50 symbols as training

ideal refereace mode

chanacl : Three ray channed model at SOHz Doppler frequency

Equaliver : fractionally spaced DFE(3 forward taps , 2 feedbeck taps). 


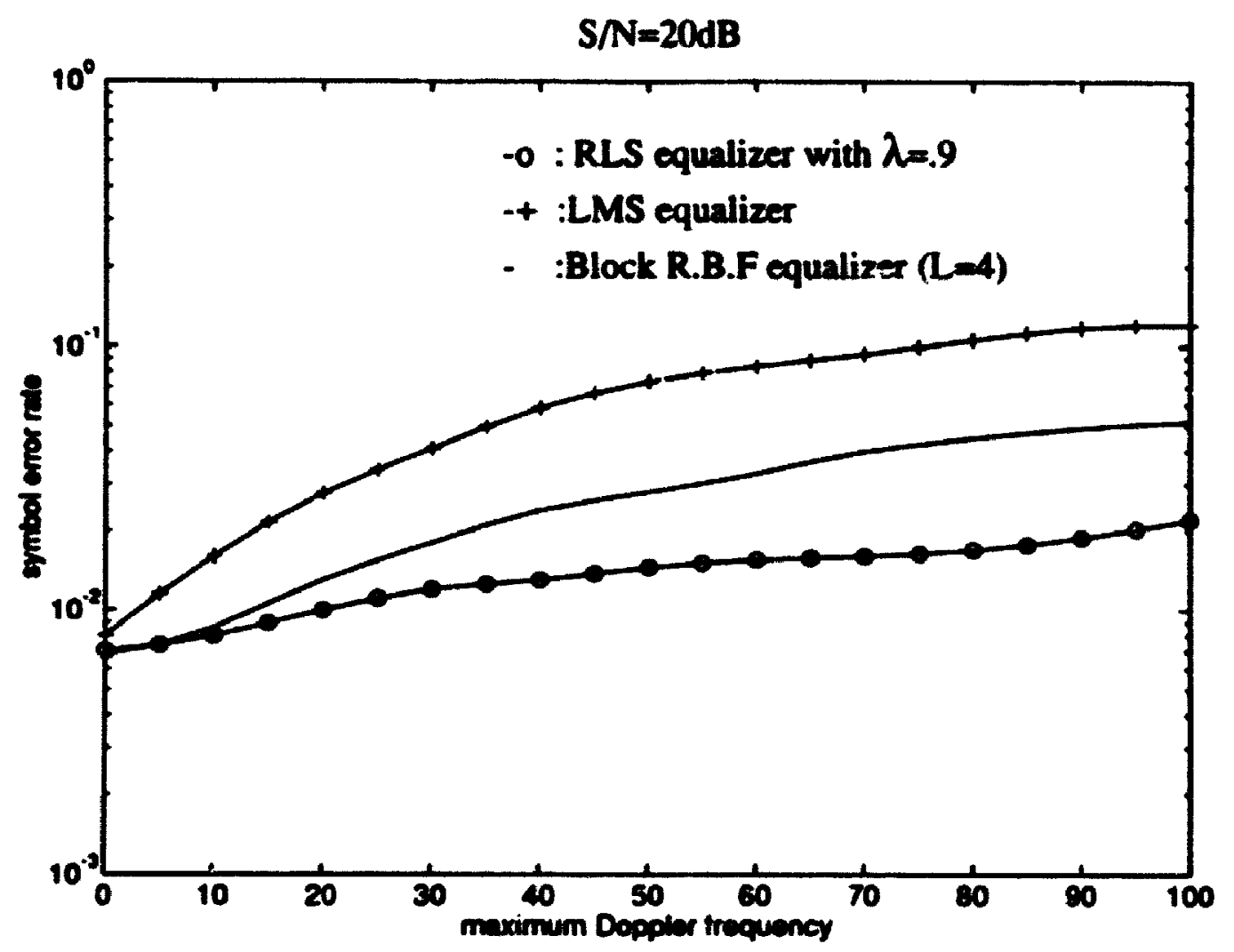

Figure (4.21) Symbol error rate Doppler frequency at three ray channel frame: 500 symbols will 50 symbols as training ideal reference mode Chanacl: Three ray chanael model Equalizer: fractionally spaced DFE(3 forward taps, 2 feedback taps). 


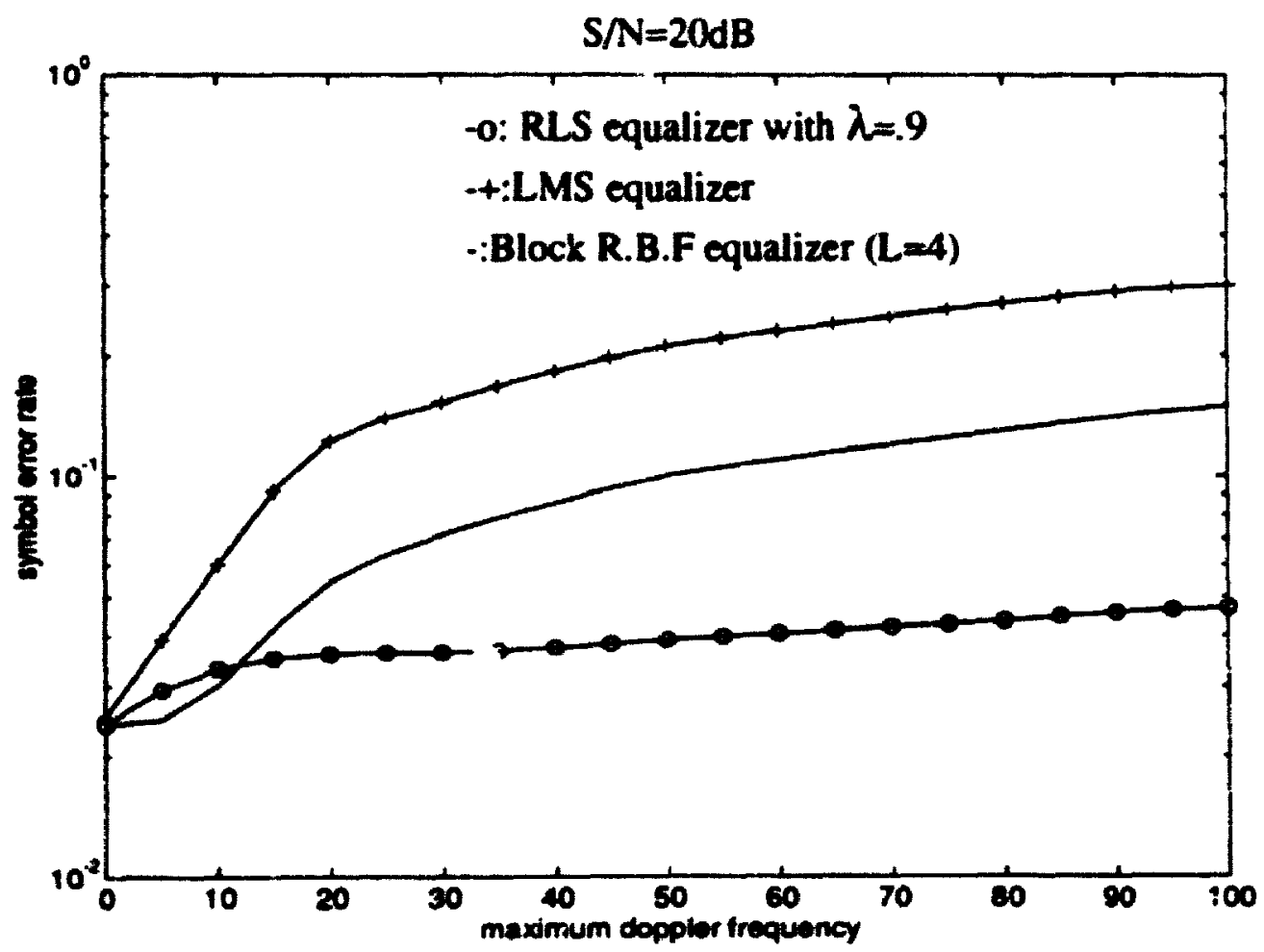

Figure (4.22) Symbol error rate /Doppler frequency at two ray channel

Prame:500 symbols with 50 symbols as training

ideal reference mode

channel: 2 equal power ray channel

Equalizer: T-spaced DFE (3 forward taps ,1 feedback tap) 


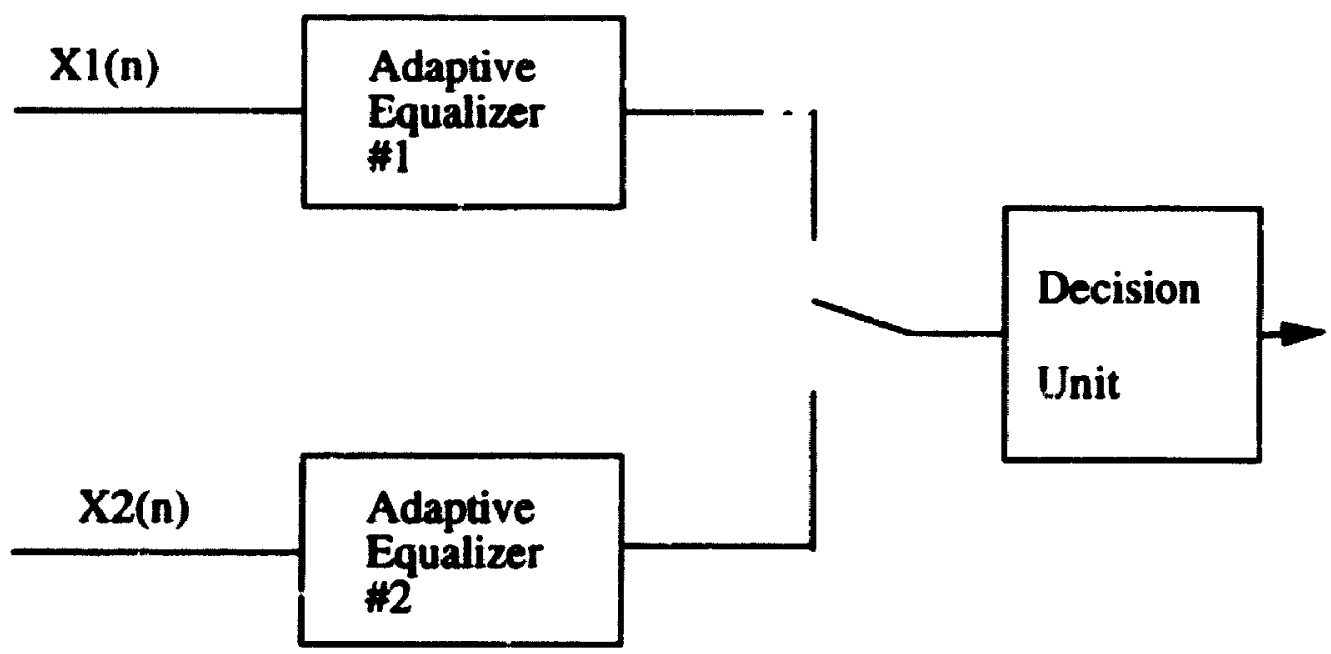

Figure (4.23) Two branch diversity with two separate adaptive equalizers. 


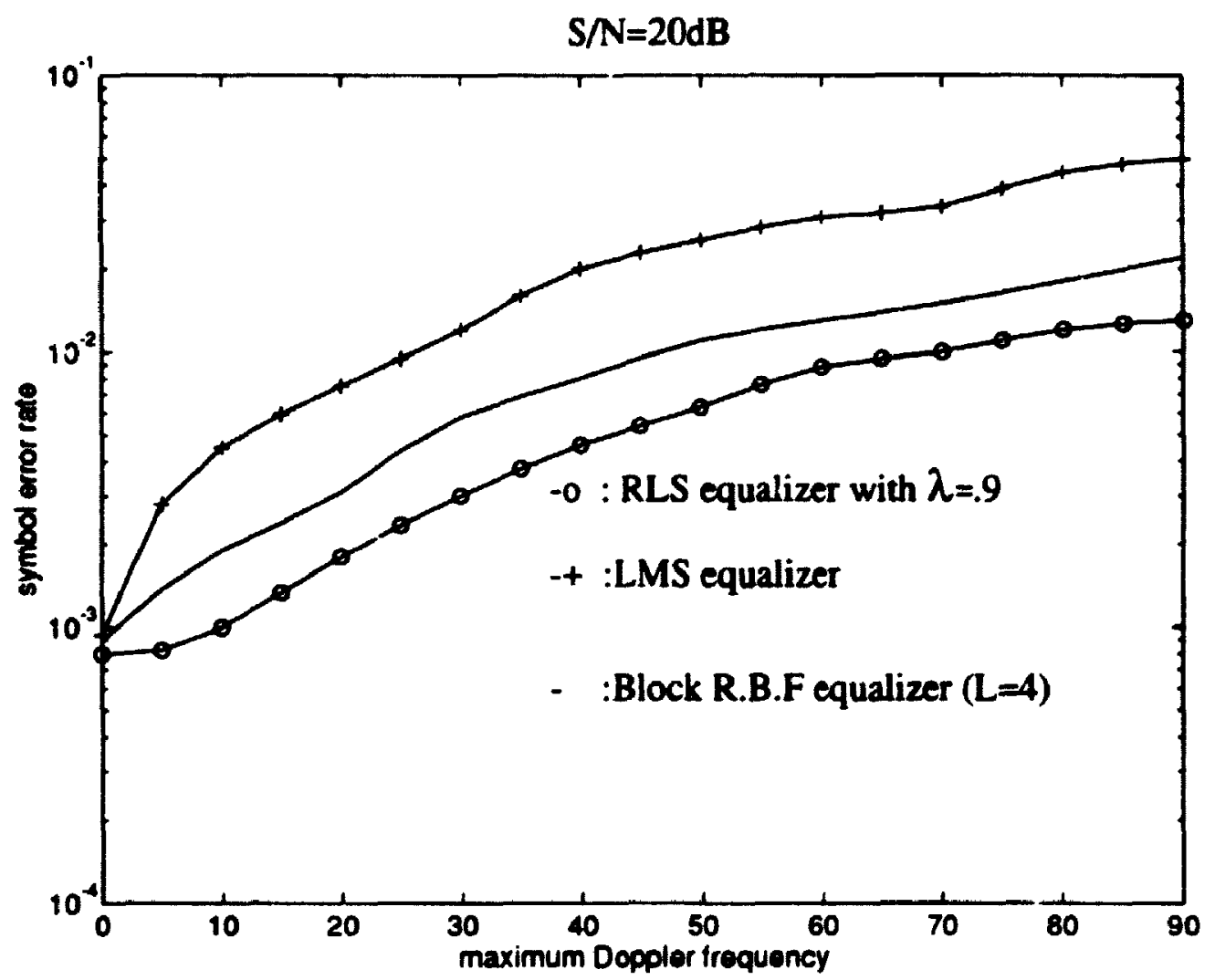

Figure (4.24) Performance of equalizer with diversity channel: 3-ray channet

Two branch diversity

Equalizer: fractionally spaced DFE (3 forward taps, 2 feedback taps). 


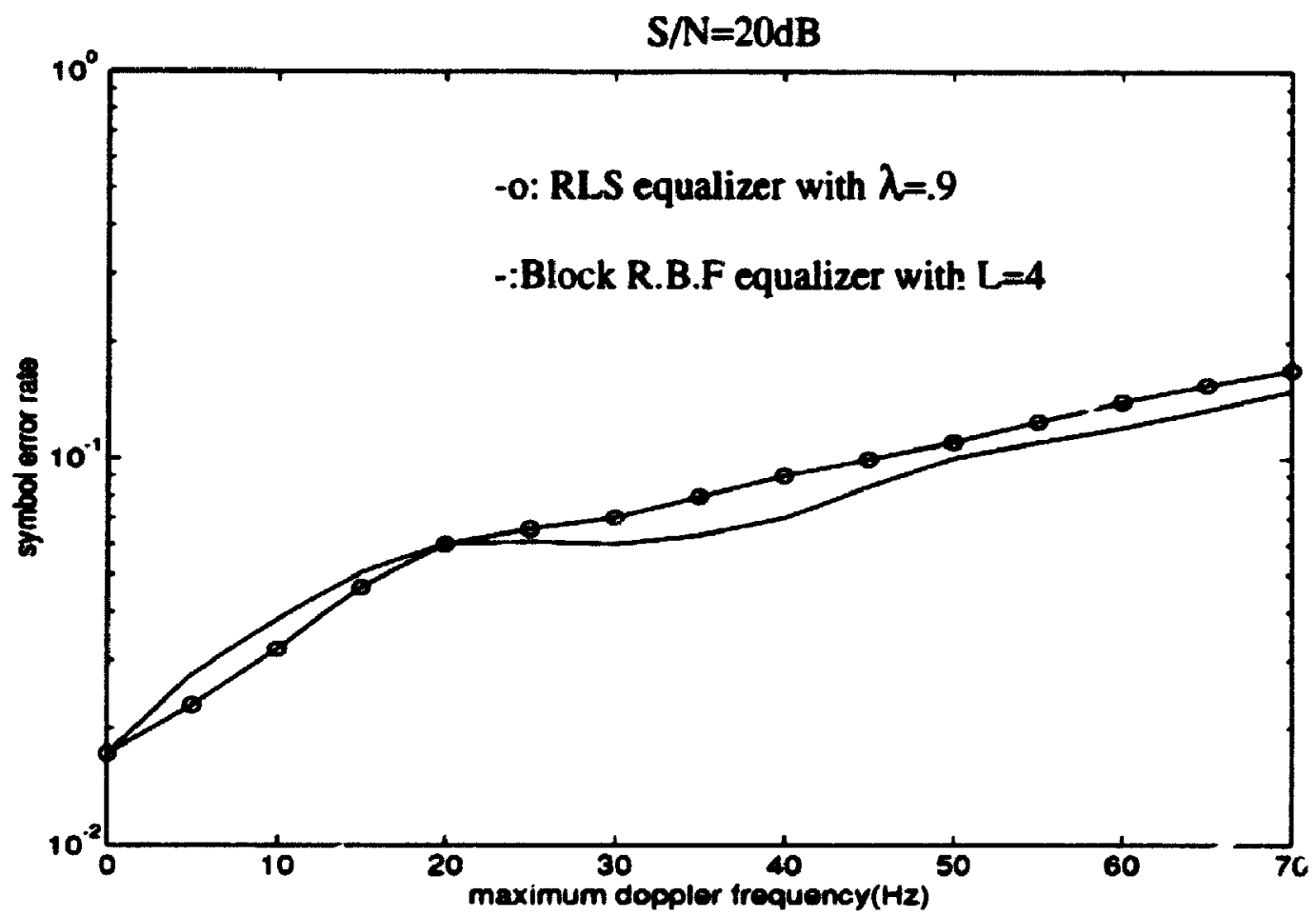

Figure (4.25) Error propagation effect

error propagation effect is investigated at different maximum doppler frequency frame:300 symbols with $\mathbf{3 0}$ symbols for training

Channel:3 ray channel model

Equalizer: fractionally spaced DFE (3 forward taps ,2 feedback taps). 


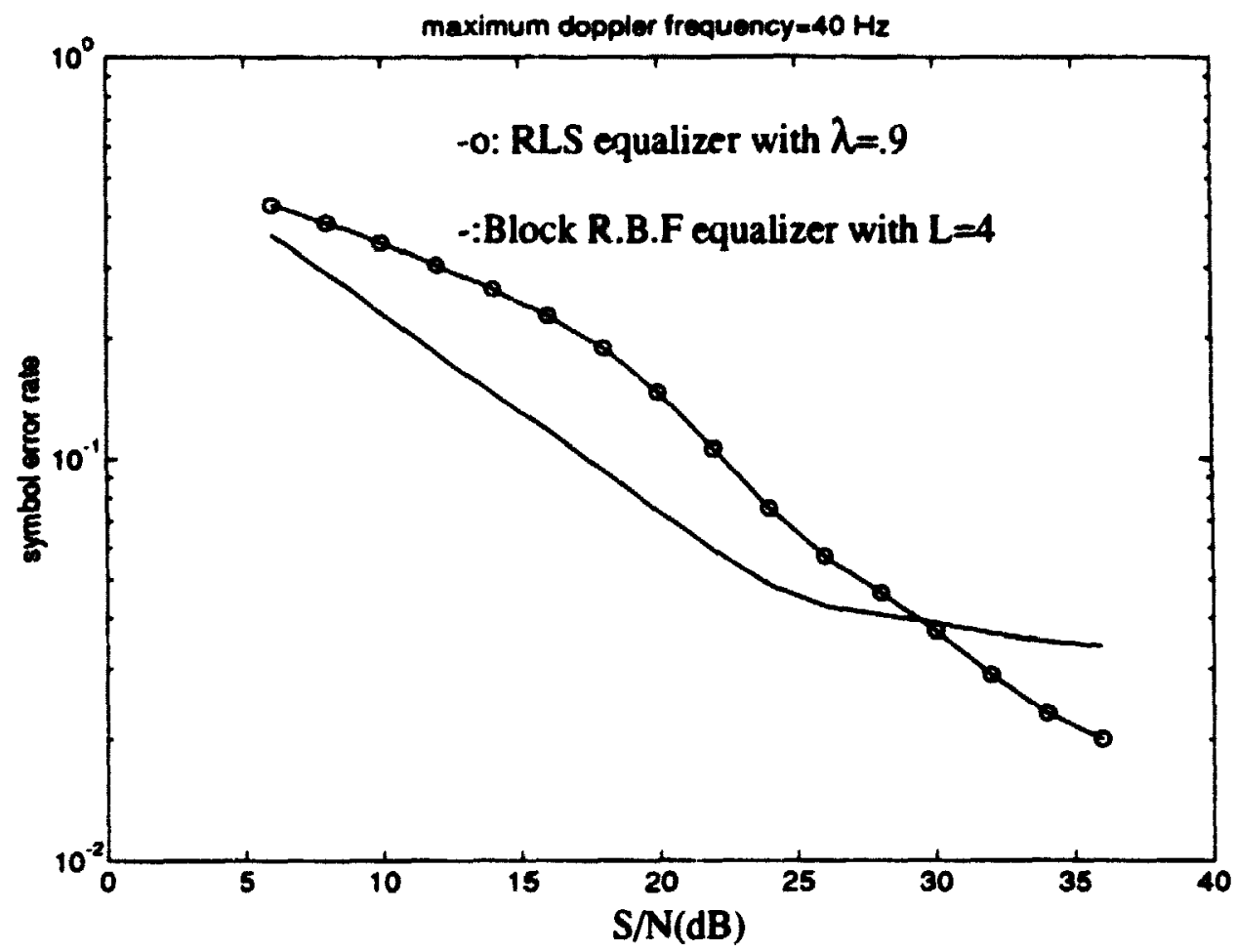

Figure(4.26) Nuise effect with considering error propagation

Frame:300 symbols with 30 symbols for training

Channel:3 ray channel modst

Equalizer: fractionally spaced DFE(3 forward taps ,2 feedback taps). 


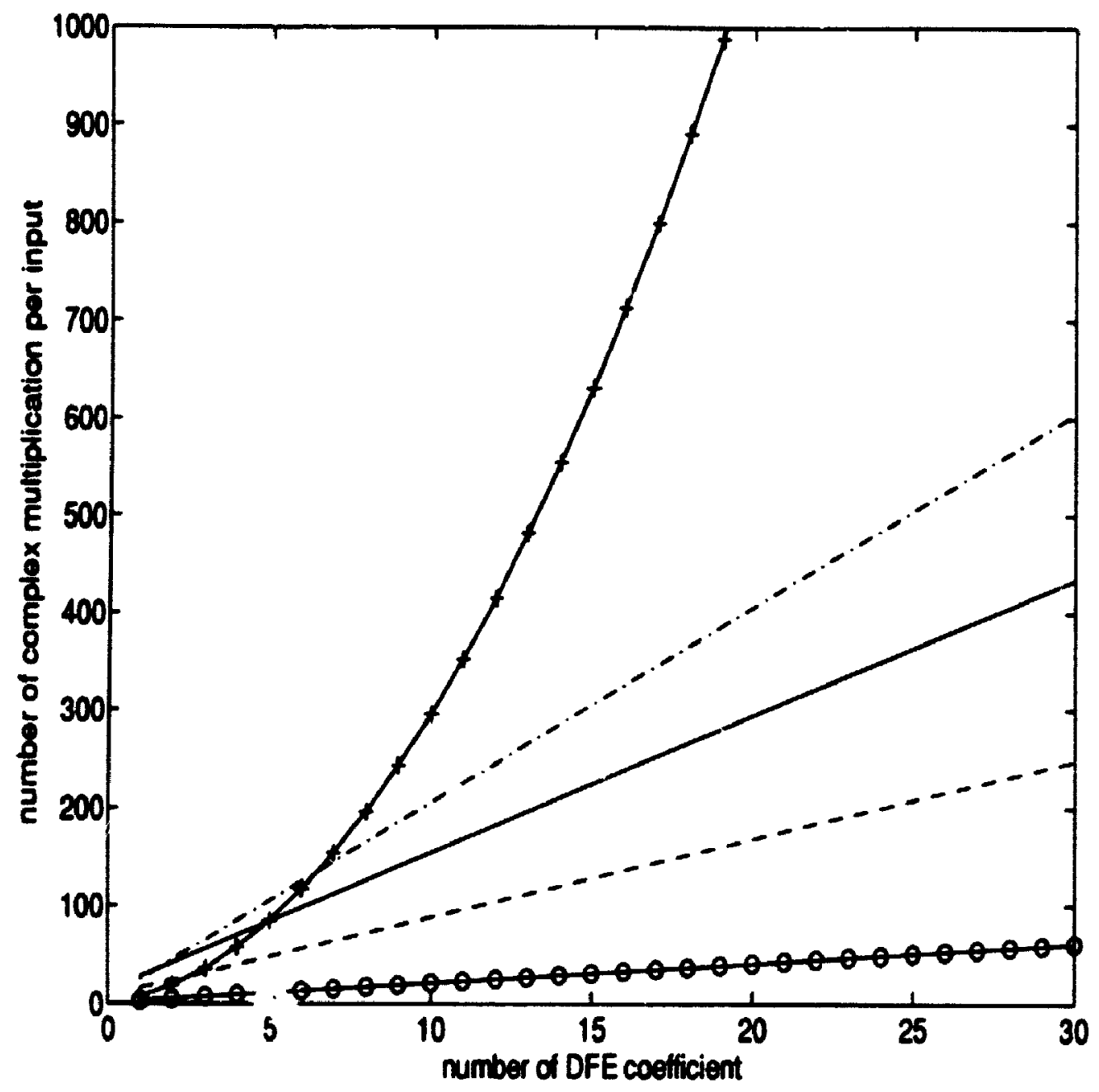

Figure (4.27) Computational complexity

\section{-.:FRLS equalizer}

-: Block radial basis function equalizer with block length:7

- - : Block radial basis function equalizer with block length:4

- 0 : LMS equalizer

-+: RLS equalizer 


\subsection{MODIFIED B.R.B.F ALGORITHM:}

To Improve B.R.B.F equalizer's performance under error propagation condition, a minor modification in B.R.B.F algorithm is introduced that we thought might improve the $B$ R.B.F performance.

In R.B.F and B.R.B.F, equalizer's adaptation rate is an increasing function of error value. This is an acceptable criteria in ideal reference mode but not in decision directed mode. The reason is that our reference is detectei data and having an increasing function of error for adaptation rate, can cause divergence. Considering this point, we incorporated the cost function introduced by Hinton [20] in to the B.R.B.F algorithm.

The combined strategy is as follow:

During training, the equalizer will be updated based on B.R.B.F algorithm, in decision directed mode instead of minimizing

we maximize

$$
T=\sum_{i=1}^{l} \exp \left(\left|\left(y_{i}-y d_{i}\right)\right|^{2} /\left(2 \sigma^{2}\right)\right)
$$

$$
T=\sum_{i=1}^{l} T_{i}
$$

where $T$ is cost function introduced by Hinton[20] as

$$
T_{i}=\sum_{i=1}^{n} \exp \left(-\left|\left(y_{\text {out }}-x d_{i}\right)\right|^{2} /\left(2 \sigma_{i}^{2}\right)\right)
$$

$y_{\text {out }}$ is equalizer ouput, $x d$ is a signal constellation point, $\sigma_{i}$ is each cluster width and $n$ is the number of points in signal constellation. For example for QPSK it is four $(n=4)$.

This cost function is depicted in figure (4.28) fo. binary PSK. 


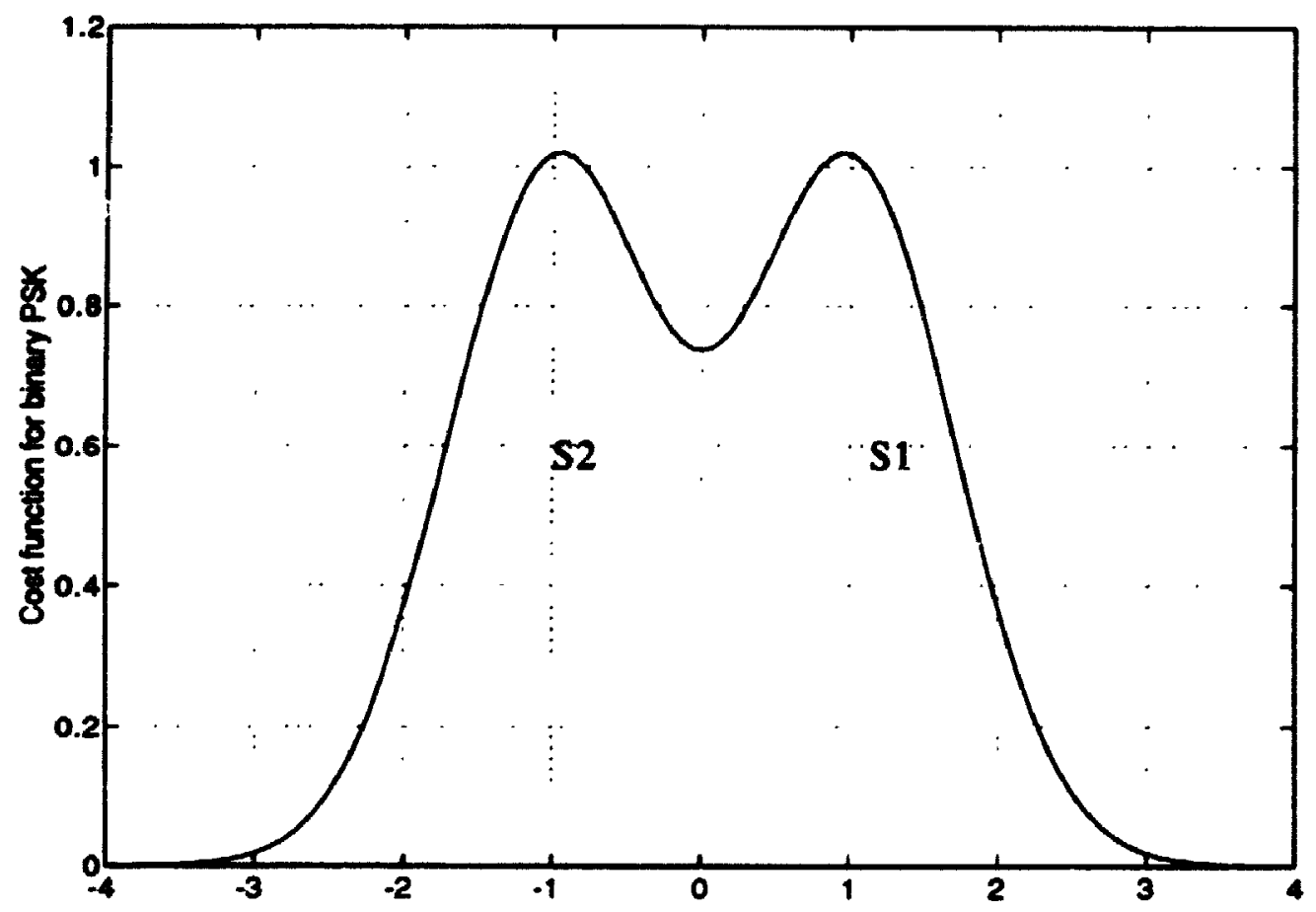

Figure (4.28) modified cost function 
The reason that we thought this equalizer could have better performance than Block radial basis function equalizer was based on the fact that, this algorithm is taking into consideration the possibility of wrong decision and tries to minimize the chance of updating the equalizer coefficients based on wrong decision.

Considering the simplest case depicted in Fig (4.28), the case of a real channel and binary PSK symbols, the equalizer weights are adapted according to the following stochastic gradient algorithm:

$$
w_{i}(k+1)=w_{i}(k)+\alpha \cdot \sum_{i=1}^{L} \frac{\partial T_{i}\left(w_{i}, y(k)\right)}{\partial w_{i}}
$$

The gradient of $\mathbf{T}_{\mathbf{i}}$ in this case becomes:

$$
\begin{aligned}
\frac{\partial T_{i}}{\partial w_{i}} & =\exp \left(\frac{-|(y(k)-S 1)|^{2}}{\left(2 \cdot \sigma^{2}\right)}\right) \cdot(y(k)-S 1) \times r(k-i) \\
& +\exp \left(\frac{-|(y(k)-S 2)|^{2}}{\left(2 \cdot \sigma^{2}\right)}\right) \cdot(y(k)-S 2) \times r(k-i)
\end{aligned}
$$

Referring to Fig 4.28 when the equalizer output $Y$ is closer tn $S 2$, the algorithm adjusts the weights in favor of $S 2$ as reflected in the second term of eq(4.49), but being in error fropagation mode, the correct decision may actually be $\mathrm{S} 1$ and the algorithm also adjusts the weights towards this decision as dictated by the first term in(4.49). It is clear that the algorithm is trying to find a compromise between the two conflicting factors.

For the modified algorithm the adaptation rate changes as follow:

When the output signal is close to the centers which means equalization almost has been done, adaptation rate is small and equalizer does the fine tuning. On the other hand, when the output is far from the centers, the derivative is almost zero so the equalizer slows down the updating at situations where most likely decision device will make a wrong decision, updating based on this wrong decision will divert equalizer from the right direction and cause divergence. When the equalizer's output is in the middle of this 
curve, derivative has the largest value, at this point the decision device can make a correct decision and having a large adaptation rate increases the convergence speed.

From above argument it can be concluded that this equalizer is working like B.R.B.F before error propagation occurs and after that it has an extra measure that could make it possible for equalizer to reconverge in a reasonable time.

Fig 4.29 and 4.30 show the fact that for a certain situation of channel (fig 4.29) the B.R.B.F equalizer could not re-converge after error propagation while the modified algorithm managed to reconverge relatively fast.

Fig(4.31) compares the B.R.B.F equalizer with its modified version for different maximum doppler frequency. From this figure, it can be concluded that the effect of this reconverging phenomenon is marginal. 

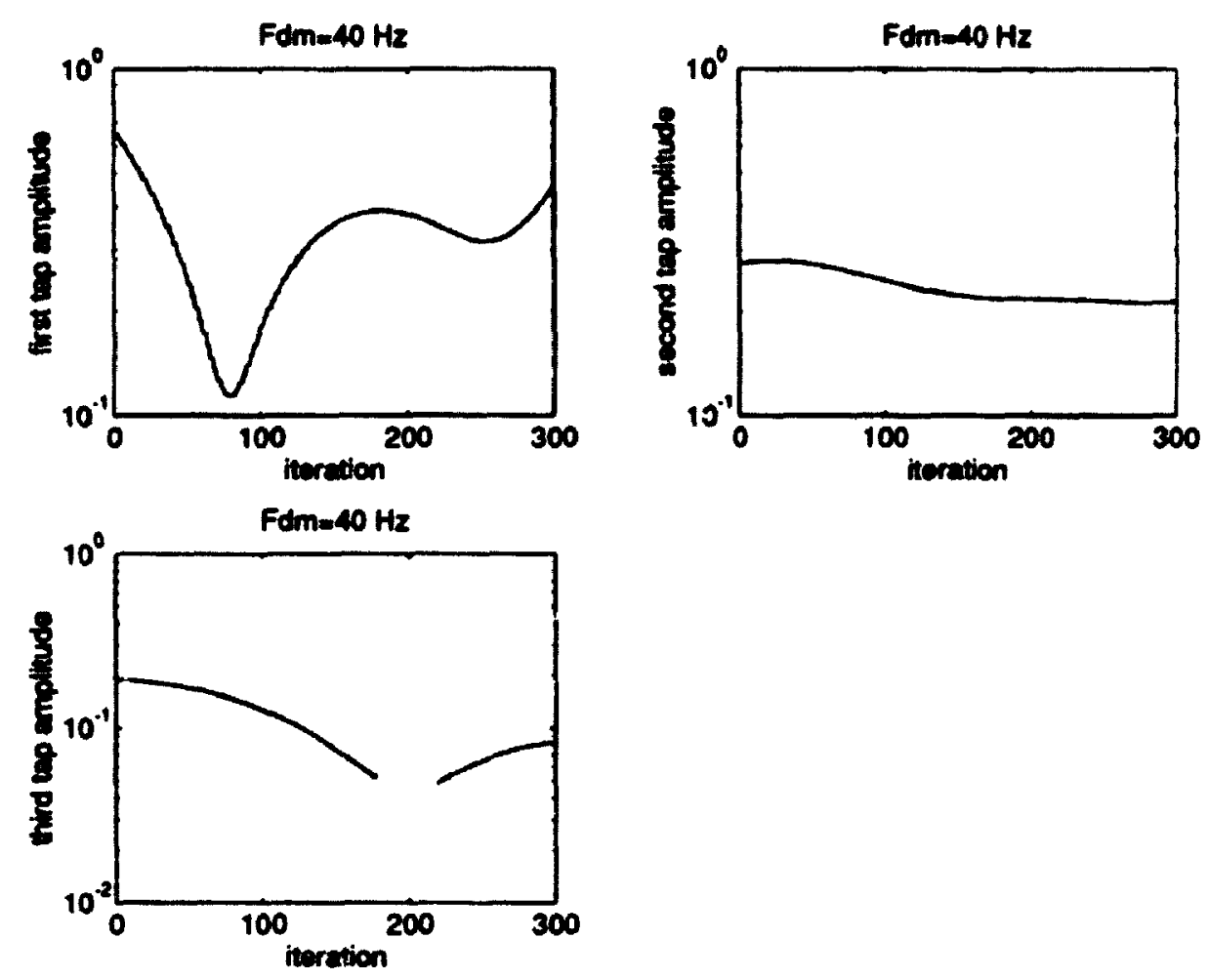

Figure (4.29) Fading channel sample function 


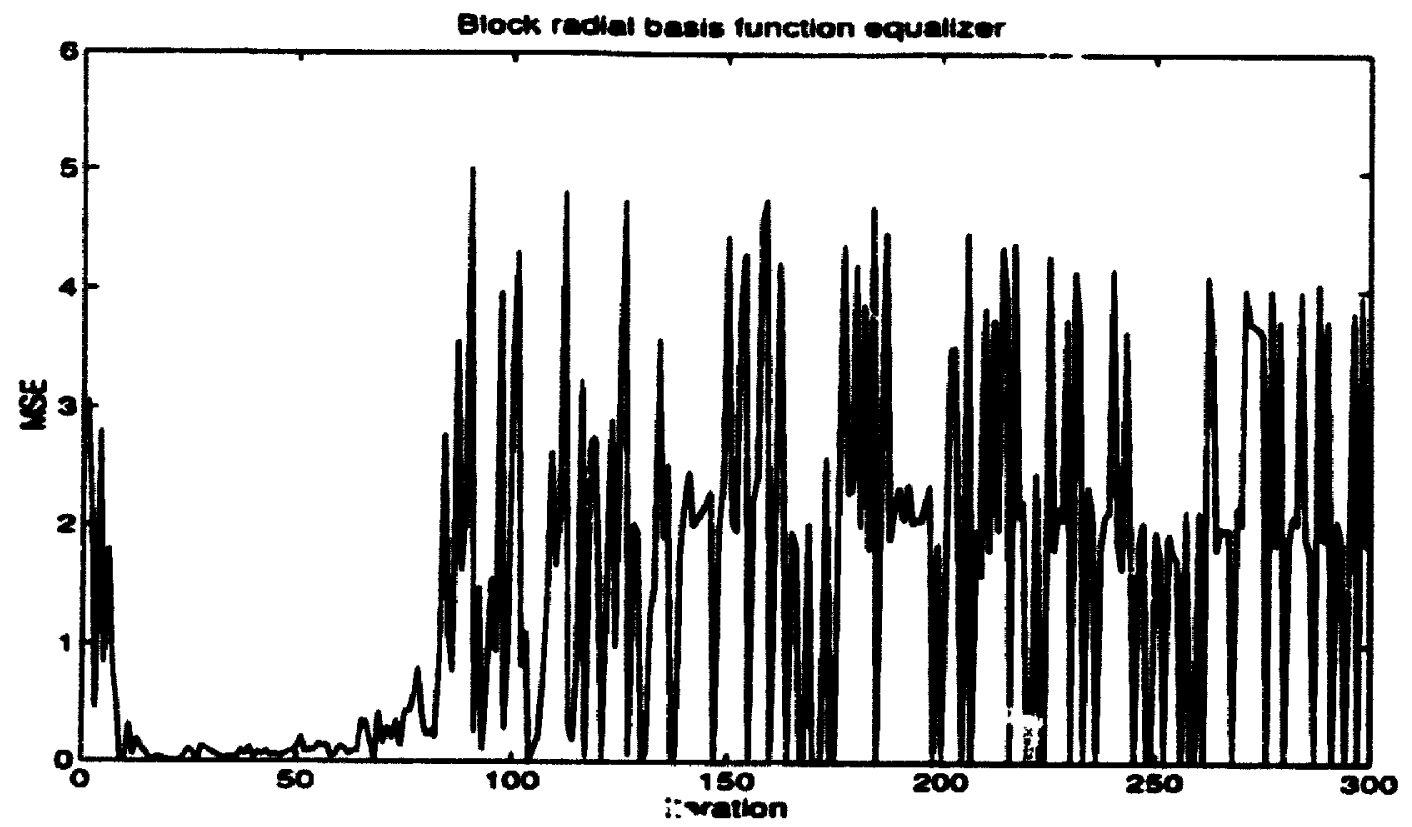

Figure (4.30) Equalizer output MSE

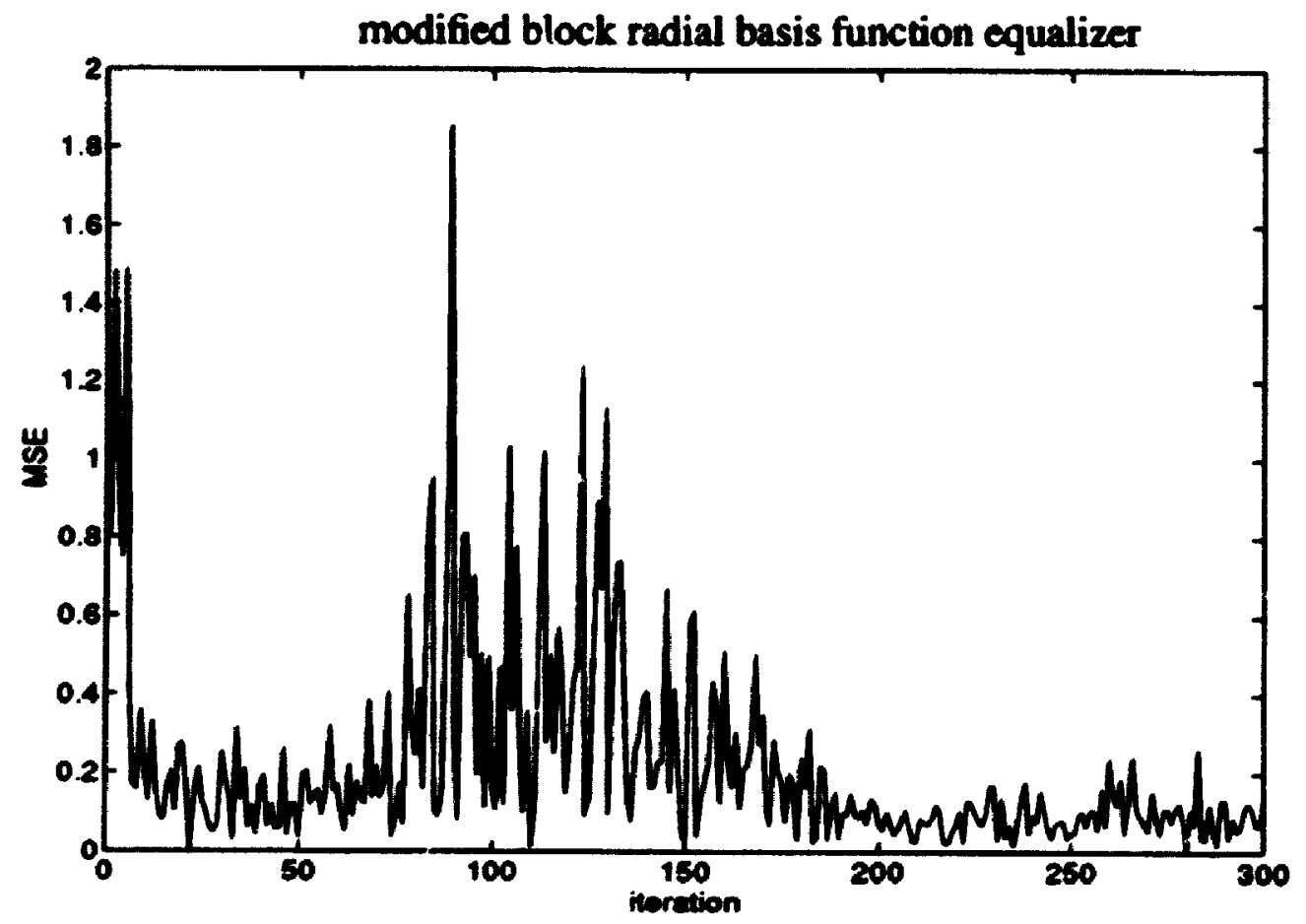




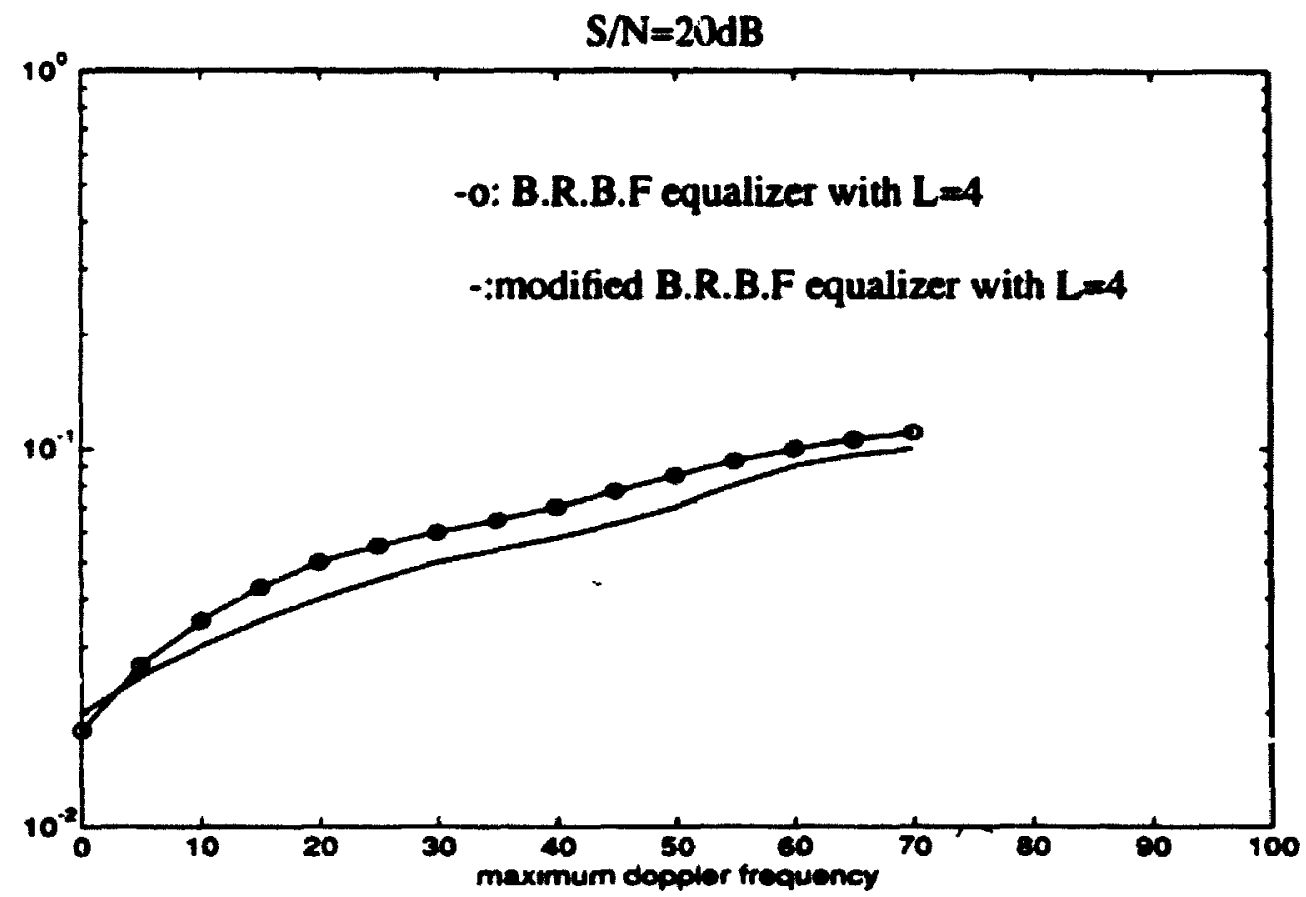

Figure (4.31) Modified B.R.B.F algorithm performance

frame:300 symbols with 30 symbols for training

Channel:3 ray channed moded

Equalizer: fractionally spaced DFE(3 forward taps, 2 feedback taps). 


\section{Chapter 5 \\ Conclusions and recommendations for future research}

In the course of this thesis research we have studied a new equalizer based on the modified concept of the radial basis function network.

We have found that this equalizer outperforms LMS equalizer considerably, with relatively small increase in computational complexity. We showed that the Block radial basis function equalize has comparable performance to the RLS equalizer in many cases (low and medium signal to noise ratio) with less complexity. The main advantage of Block radial basis function equalizer over RLS equalizer is the fact that it doesn't suffer from numerical instability.

Through the analysis of radial basis function equalizer insight is offered about the convergence speed of this algorithm. It has been shown that for a given level of steady state error, convergence speed is increased by up to fifty percent compared to LMS equalizer.

An important feature of the new algorithm is that approximate formulas can be derived to predict the convergence speed and misadjustment factor in both stationary and nonstationary environments. These theoretical predictions agree well with simulations of the algorithm.

The main disadvantage of Block radial basis function equalizer is it's sensitivity to channel covariance matrix eigenvalue spread. Although there is no clear ielationship between eigenvalue spread and block RBF equalizer performance, it is obvious that equalizer parameters have to be adjusted based on channel characteristics(maximum value for equivalent adaptation rate is imposed by stability condition for equalizer which is related to channel characteristics).

Our attempt to modify Block radial basis function equalizer to enable it to cope with error propagation was not a successful approach: considering it's marginal improvement and considerable computational complexity, it is a subject that needs more investigation. 
Understanding the relation between the channel covariance matrix eigenvalue spread and Block R.B.F equalizer performance is another issue that is worth of further investigation.

During this thesis it has been established that Block R.B.F equalizer is a low complexity gradient based adaptive filter with performance similar to RLS equalizer. Therefore it is important to investigate applicability of this filter in other applications like echo canceling.

Using this equalizer as part of more elaborated receiver structure for mobile communication with coding and diversity and optimal channel combining, is another issue for further study. The possibility of reducing the number of necessary diversity branches (for a given bit error rate) in return of having more effective equalizer can he a significant result of using this equalizer.

It is known that updating equalizer coefficients based on estimated channel, by computing Weiner solution, results in bet.er performance for equalizer than direct adaptation. Applying Block R.B.F algorithm as channel estimator and updating equalizer coefficients based on estimaied channel is another subject for further study. 

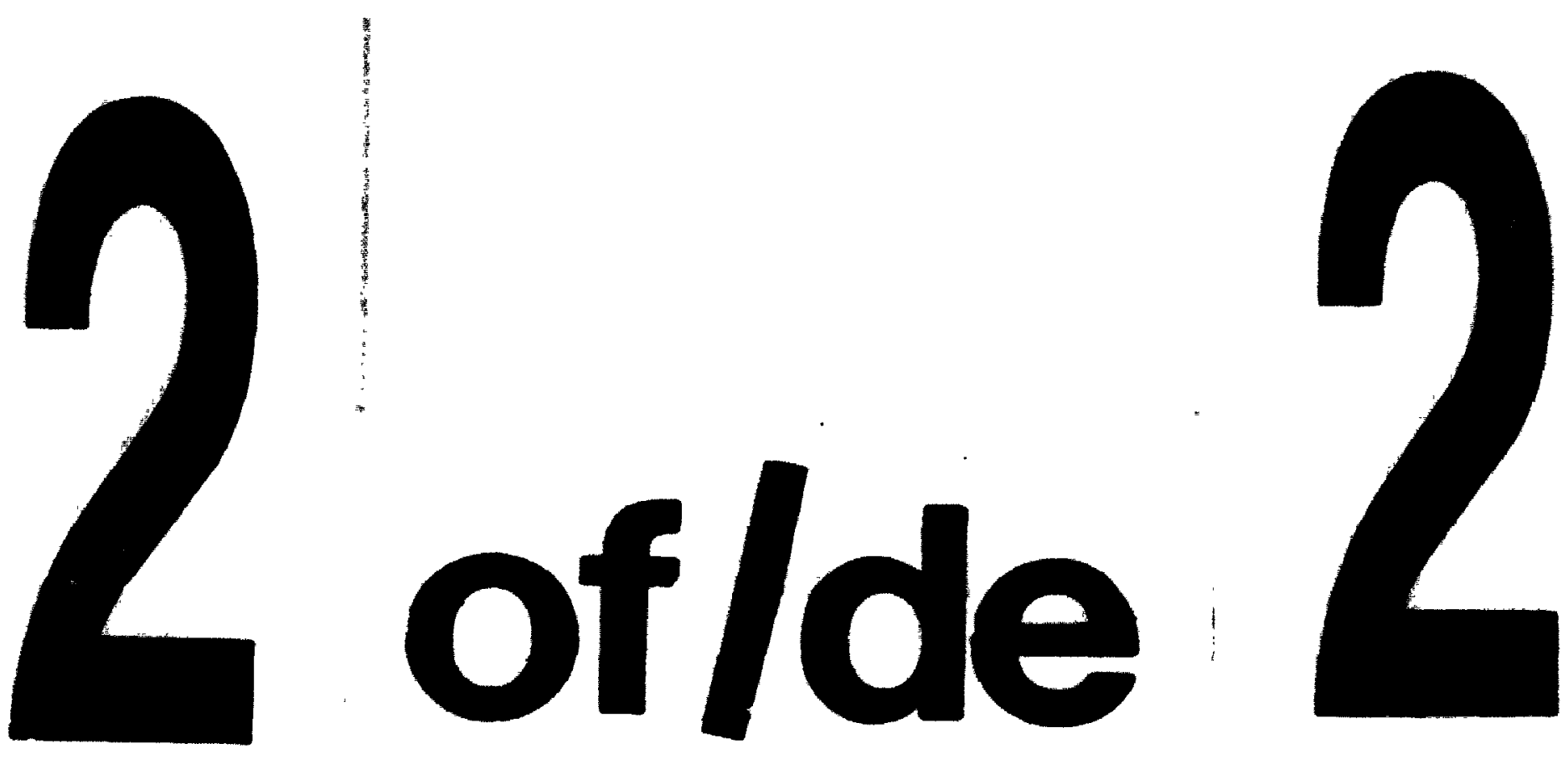

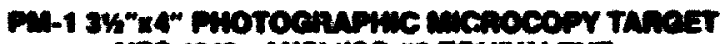

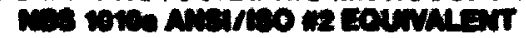

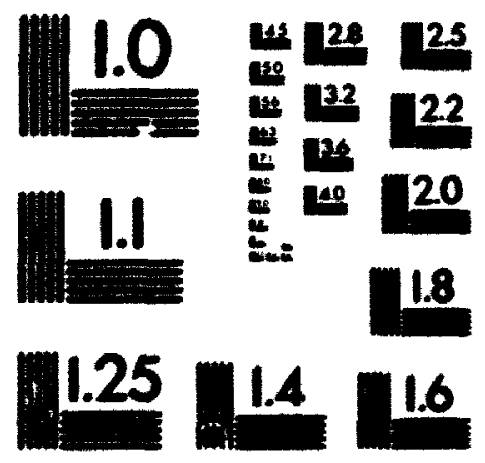

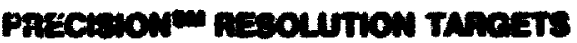




\section{REFERENCES:}

[1] A. S. Acampora \& j. H. Winters, "System application for wireless Indoor communication", I.E.E.E Comm . Mag, vol. 25, no. 8, pp. 11-20, Aug. 1987

[2] E. F. Casas \& C. Leung , "A simple digital fading simulator for mobile radio", Proc. VTC 88, pp. 212-217, sept. 1988

[3] J. Chao \& H. Pepez, "A fast adaptive filter algorithm using eigenvalue reciprocals as step sizes", IEEE Trans. on ASSP. no:8, pp. 1343-1351, Aug 1990

[4] S. Chen," Recursive hybrid algorithm for non-linear system identification using radial basis function networks", Int. journal of control. pp. 1051-1070, may 1992

[5] S. Chen, "Application of radial basis function network", I.E.E Proceeding -F, vol. 139, no.6, pp. 378-384, December 1992

[6] S. Chen, "Orthogonal least squares learning algorithm for radial basis function net works", IEEE Trans. on neural network, vol.2, no. 2, pp. 302-308, March 1991

[7] S. Chen \& S. McLaughlin,"Reduced-Complexity multi-stage blind clustering equal izer", ICC93, pp. 1149-1153

[8] S. N. Crozier, "Short-Block data detection techniques employing channel estimation for fading,Time-dispersive channels", Ph.D. Thesis, Dept. of systems and computer Engineering, Carleton University, Ottawa, ON, Canada, Jul. 1986

[9] J. Doherty, "A row sction projection algorithm for adaptive filtering", In Proc. IEEE Int ConfAcoustic Speech,Signal Processing, pp. 1397-1400, April 1990

[10] J. Doherty \& R. Mammone, "An adaptive algorithm for stable Decission-Feedback 6ilering", IEEE Trans. on Circuits and Systems, vol40, no.1 pp. 1-9, January 1993

[11] N. Dyn, "Interpolation of scattered data by radial functions", Topics in multivariate approximation, Academic Press, New-York, 1987

[12] ETSL/GSM Recommendations, series 05 
[13] EIAVTIA, Ceilular System: Dual-mode subscriber Equipment-Network Equipment Compatibility Specifications, IS-54, Jan 1991

[14] F. Fleftheriou \& D. D. Falconer, “Adaptive equalization techniques for HF channels", IEEE journal on selected areas in communications, no.2, pp. 238-246 February 1987

[15] E. Eleftheriou \& D. D. Falconer, "Tracking properties and steady state performance of RLS adaptive filter algorithms", IEEE Trans. ASSP, no.5, pp. 1097-1109, Oct 1986

[16] K. Funahashi, "On the the approximate ation of continuous mappings by neural networks", neural networks, vol 2, pł. 183-192, 1989

[17] G. Gibson \& N. Cowan,'The application of Non linear structures to the reconstruction of binary signals", IEEE Trans, on signal processing , vol.39, no 8, pp. 1877-1884, August 1991

[18] D. J. Goodman ,"Second generation wireless Information networks", IEEF Trans. vehic .Tech. vol 40, no.2, pp.366-374, May 1991

[19] R. Harris \& D. Chabries \&A. Bishop."A variable step adaptive filter algorithm", IEEE Trans. On Acoustics Speech and Signal Processing, vol. ASSP-34, no. 2 , pp. 309-316, April 1986

[20] G. Hinton, "The boot strap Widrow rule as a cluster formation Algorithm", Neural computatoin, pp.355-362, June 1990

[21] G. Hinton, "Soft decission directed equalizer", IEEE Trans. on communication, pp 275-279, Feb.1993

[22] L. L. Horowitz \& K. Senne, "Performance advantage of complex LMS for controlling narrow-band adaptive arrays", IEEE Trans. on Acoustics,Speech and Signal Processing, vol. ASSP-29, NO.3, pp. 722-736, June 1981

[23] J. Y. Hui, "Bit-Rate Limitations of the digital Radio Channel Due to Multipath Intersymbol Interferemce and fading", in proc Globecom 87, Pp.48.4.1-7, May 1987 
[24] W. C. Jakes, "Microwave Mobile Communications", New York, NY Wiley, 1974

[25] J. Karaoguz \& S. Ardalan, "A soft decission-directed blind equalization algorithm applied to equalization of mobile communication channels", ICC 92, pp. 1272 1276 , Sept. 1992

[26] R. H. Kwong \& E. W.Johnston, "A variable step size LMS Algorithm", IEEE Trans. on signal processing, vol40 , No.7, pp. 1632-1642, July 1992

[27] A. Leclert \& P. Vandamme, " Decission feedback equalization of dispersive Radio channels", IEEE Trans.on communication, vol.com. 33, no. 7, pp. 676-684, July 1985

[28] W. C. Lee, "Mobile communications Engineering", New York, NY McGraw Hill, 1982

[29] Yow-Jong Liu,“Bi-directional equalization technique for TDMA communication system over land mobile radio channels", Proc. Globe Com., Phoenix AZ, pp. 1458-1462, December 1991

[30] Norm W. K .Lo,"adaptive equalization and diversity combinning for a mobile radio channer", Master thesis, Dept. of systems and computer Engineering. Carieton University, Ottawa, ON, Canada, July 1990

[31] S. Marcos \& O. Macchi, "Tracking capability of the least mean square algorithm", IEEE Trans on Acoustics Speech and Signal Processing vol. ASSP-35, no.11 Pp. 1570-1578, November 1987

[32] V. John Mathews \& Z. Xie, "Stochastic gradient adaptive filter with gradient adaptive step size", 1990 ICASSP

[33] J. E. Mazo, "On the Independence theory of equalizer convergence", The BELL System Technical Journal, pp. 963-993, May 1979

[34] J. Park. "Universal approximation using radial basis function networks", Neural computation, 1991, pp. 246-257

[35] T. Poggio, "Network for approximation and learning", Proc. of the I.E.E.E, vol.78, no.9. pp. 1481-1495, September 1990 
[36] M. J. D. Powell, "Radial basis functions for multivcriable interpolation", algorithms for approximation, Clarendon Press, Oxford, 1987

[37] J. G. Proakis, “Adaptive Equalization for TDMA Digital Mobile Radio", I.E.E.E Trans. on Vehicular Techhnology, vol. 40, No.2, pp. 333-341, May 1991

[38] J. G. Proakis, "Digital Communication", New-York, McGraw-Hill, 1983

[39] S. U. H. Qureshi, "Adaptive Equalization", Proc. of the I.E.E.E, vol. 73, no.9, pp 1349-1387, Sept. 1985

[40] K. Raith \& J. Uddenfeldt, "Capacity of digital cellular TDMA systems", IEEE Trans.vehic .Tech, vol. VT.40, pp. 323-332, May 1991

[41] S. Renals and $\mathbf{R}$. Rohwer "Phoneme classifiention experiments using radial basis functions", Proceedings of the international joint conference on newral networks, pp.1-461 :1-467, Washington,D.C, June 1989.

[42] T. J. Shan \& T. Kailath,"Adaptive algorithm with an automatic gain control feature". IEEE Trans. on circuits and systems, vol. 35, No.1. pp. 122-127. Jan 1988

[43] W. T. Webb, “Dispersive mobile radio channels", IEE Proceedings, vol.138, no.6 pp. 566-576, December 1991

[44] B. Widrow, "Stationary and nonstationary learning characteristics of the LMS adaptive filver", Proc of the I.E.E.E, vol. 64, no. 8, pp. 1151-1161, August 1976

[45] G. Ybarra \& S. Alexander, "Effects of iil-conditioned data on least squares adaptive filters", IEEE ICASSP, pp.1387-1390, 1988 


\section{APPENDIX A:}

Steady state behavior analyis in time invariant channel

This analysis is similar to [22] for a fixed rate adaptive filter. In my work I modified that analysis for Radial basis function algorithm.

In the following analysis excess MSE has been computed in terms of average value and second order moment of equivalent adaptation rate, then these moments are computed in terms of variance of cluster and $\beta$.

In order to derive a closed form expression for excess mean square error we conisdered the gaussian distribution for input vector. This assumption has been made implicitly or explicitly almost in all papers related to computing the steady state error for equalizer [22][19][31].

Up-dating equation is:

$$
W(k+1)=W(k)+\beta_{e q}(k) \cdot \operatorname{err}(k) \cdot x_{k}^{*}
$$

or

$$
\begin{gathered}
W(k+1)=W(k)+\beta_{e q}(k) \cdot\left(Y_{d}(k) X_{k}^{*}-X_{k}^{*} \cdot x_{k}^{T} \cdot W(k)\right) \\
W(k+1)=\left[I-\beta_{e q}(k) \cdot X_{k}^{*} \cdot X_{k}^{T}\right] \cdot W(k) \\
+\beta_{e q}(k) \cdot Y_{d}(k) X_{k}^{*}
\end{gathered}
$$

It is convenient to write above equation in terms of error weight vector which is $W^{f}(k)=W(k)-W_{\text {opt }}\left(W_{\text {opt }}\right.$ is the Weiner solution for the filter weight vector $)$

$$
W^{*}(k+1)=W(k+1)-w_{\text {opt }}
$$




$$
\begin{gathered}
W^{e}(k+1)=W(k)+\beta_{e q}(k) \cdot X_{k}^{*} \cdot \operatorname{err}(k)-W_{o p t} \\
W^{e}(k+1)=W(k)-W_{e p t}+\beta_{e q}(k) \cdot X_{k}^{*} \cdot\left[Y_{d}(k)-X_{k}^{T} \cdot W(k)\right] \\
W^{e}(k+1)=W^{e}(k)+\beta_{e q}(k) \cdot X_{k}^{*} \cdot\left[X_{k}^{T} \cdot W_{o p t}+e_{k}\right]- \\
\beta_{e q}(k) \cdot X_{k}^{*} \cdot X_{k}^{T} \cdot W(k)
\end{gathered}
$$

where $y_{d}(k)$ desired symbol $=X_{k} T W_{\text {opt }}+e_{k}$ has been used in above equation ( $e_{k}$ is zero mean gaussian noise obtained by utilizing the weight vector $\mathbf{W}_{\text {opt }}$ on the data at time $k T$ )

$$
\begin{aligned}
W^{e}(k+1)= & {\left[I-\beta_{e q}(k) \cdot X_{k}^{*} \cdot X_{k}^{T}\right] \cdot W^{e}(k) } \\
& +\beta_{e q}(k) \cdot X_{k}^{*} \cdot e_{k}
\end{aligned}
$$

let $\mathbf{R}$ be channel covariance matrix :

$$
R=E\left[X_{k}^{*} \cdot X_{k}\right]
$$

Since $R$ is symmetric it may be rotated into a diagonal matrix by a unitary transformation

$$
R=Q \cdot \Lambda \cdot 2^{-1}
$$

where $Q$ is the orthonormal modal matrix of $R$.

By introducing rotated coordinates with premultiplying all vectors by $Q^{-1}$ we define:

$$
\left\{\begin{array}{c}
W_{o p t}=Q^{-1} \cdot W_{o p t} \\
W^{e}(k)=Q^{-1} \cdot W^{e}(k) \\
X(k)=Q^{-1} \cdot X_{k} \\
\hat{X C}(k)=Q^{-1} \cdot X_{k}^{*}
\end{array}\right.
$$


Equation for error weight vector in new coordinate system becomes:

$$
\begin{gathered}
\hat{W}^{e}(k+1)=\left[I-\beta_{e q}(k) \cdot \hat{x} C(k) \cdot \hat{x}^{T}(k)\right] \cdot \hat{W}^{e}(k)+ \\
\beta_{e q}(k) \cdot e_{k} \cdot \hat{X} C(k)
\end{gathered}
$$

One more point has to be proved before deriving an expression for MSE:

statement:

$$
E\left[e_{k} \cdot \hat{X} C(k)\right]=0
$$

proof:

$$
\begin{gathered}
e_{k}=Y_{d}(k)-\hat{X}^{T}(k) \cdot \hat{W}_{o p t} \\
E\left[e_{k} \cdot \hat{X} C(k)\right]=E\left[\hat{X} C(k) \cdot Y_{d}(k)\right]-E\left[\hat{X} C(k) \cdot \hat{X}^{T}(k) \cdot \hat{W}_{o p t}\right]
\end{gathered}
$$

recalling that:

$$
\begin{gathered}
W_{o p t}=R^{-1} \cdot P \\
W_{o p t}=Q^{-1} \cdot R^{-1} \cdot Q \cdot Q^{-1} \cdot P \\
\Lambda^{-1} \cdot P
\end{gathered}
$$

where

$$
P=E\left[X^{*} \cdot Y_{d}(k)\right]
$$

$\Lambda$ is a diagonal matrix with elements equal to eigenvalues of covariance matrix so

$$
E\left[e_{k} \cdot \hat{X} C(k)\right]=\hat{P}-\left(\Lambda \cdot \Lambda^{-1} \cdot \hat{P}\right)=0
$$


This means $X(k)$ is stochastically orthogonal to $q$.To compute MSE we write MSE(k) as:

$$
\begin{aligned}
& \operatorname{MSE}(k)=E\left[\left(\varphi_{k}+e \pi(k)-e_{k}\right)^{2}\right] \\
& \operatorname{MSE}(k)=e r r_{\text {min }}+2 \cdot E\left[e_{k} \cdot\left(\operatorname{err}(k)-e_{k}\right)\right]+ \\
& E\left[\left(\operatorname{err}(k)-e_{k}\right)^{2}\right] \\
& \operatorname{MSE}(k)=\operatorname{err}_{\min }-2 \cdot E\left[e_{k} \cdot \hat{\boldsymbol{X}}^{T}(k) \cdot \hat{\boldsymbol{W}}^{e}(k)\right]+ \\
& E\left[\left[\hat{w}^{e^{*}}(k)\right]^{T} \hat{x} C(k) \cdot \hat{x}^{T}(k) \cdot \hat{w}^{*}(k)\right]
\end{aligned}
$$

Independent input vector assumption and orthogonality of $q_{z}$ and $X(k)$ can be used to simplify this equation as [22]:

$$
M S E(k)=e r r_{\text {min }}+1^{T} \cdot G(k)
$$

' 1 ' is a one-column vector with elements equal to' 1 ' and $G(k)$ is diagonal terms of the matrix

$$
\Lambda \cdot E\left[w^{e}(k) \cdot\left[w^{e^{*}}(k)\right]^{T}\right]
$$

Obviously the next step in computing MSE(k) is finding

$$
E\left[w^{*}(k) \cdot w^{*}(k)^{T}\right]
$$




$$
\left(\begin{array}{c}
\hat{w}^{e}(k+1) \cdot\left[\hat{w}^{*}(k+1)\right]^{T}=\left[I-\beta_{e q}(k) \cdot \hat{X} C(k) \cdot \hat{X}^{T}(k)\right] \cdot \hat{w}^{e}(k) \\
{\left[\hat{w}^{*}(k)\right]^{T}\left[I-\beta_{e q}(k) \cdot \hat{X}(k) \cdot \hat{X} C^{T}(k)\right]^{+}} \\
{\left[I-\beta_{e q}(k) \cdot \hat{X} C(k) \cdot \hat{X}^{T}(k)\right] w^{e}(k)\left[\beta_{e q}(k) \cdot \hat{X}^{T}(k) \cdot e_{k}\right]} \\
{\left[\beta_{e q}(k) \cdot \hat{X C}(k) \cdot e_{k}\right]\left[\hat{w}^{*}(k)\right]^{T}\left[I-\beta_{e q}(k) \cdot \hat{X}(k) \cdot \hat{X} c^{T}(k)\right]} \\
+\beta_{e q}^{2}(k) \cdot e_{k}^{2} \cdot \hat{X} C(k) \cdot \hat{X}^{T}(k)
\end{array}\right.
$$

For computing the expected value of above equation we notice that by the independence assumption of input vector and stochastic orthogonality of $c_{k}$ and input vector, all the cross terms will be eliminated, thus the expected value of above equation simplifies to:

$$
\begin{aligned}
& E\left[\hat{W}^{e}(k+1) \cdot\left[\hat{w}^{e}(k+1)\right]^{\top}\right]=E\left[\hat{w}^{e}(k) \cdot\left[\hat{w}^{*}(k)\right]^{T}\right] \\
& -E\left[\beta_{e q}(k)\right] \cdot \dot{\Lambda} \cdot \dot{E}\left[\hat{W}^{e}(k) \cdot\left[\hat{w}^{*}(k)\right]^{T}\right] \\
& -E\left[\beta_{e q}(k)\right] \cdot E\left[\hat{w}^{e}(k) \cdot\left[w^{e}\right]^{T}\right] \cdot \Lambda \\
& +E\left[\beta_{e q}^{2}(k)\right] \cdot E\left[\hat{X C}(k) \hat{X}^{T}(k) \cdot A \cdot \hat{X}(k) \cdot \hat{\left.X C^{T}(k)\right]}\right. \\
& +E\left[\beta_{e q}^{2}(k)\right] \cdot \operatorname{err}_{\min } \cdot \Lambda
\end{aligned}
$$

for notational simplicity we defined $A$ as:

$$
A=E\left[W^{e}(k) \cdot\left[w^{*}(k)\right]^{T}\right]
$$


Based on gaussian factoring theory we have(for complex gaussian random variable)

$$
\begin{aligned}
E[Z 1 \cdot \bar{Z} 2 \cdot Z 3 \cdot \bar{Z} 4]= & E[Z 1 \cdot \bar{Z} 2] \cdot E[Z 3 \cdot \bar{Z} 4]+E[Z 1 \cdot \bar{Z} 4] \\
& E[\bar{Z} 2 \cdot \bar{Z} 3]
\end{aligned}
$$

So

$$
E\left[\beta_{e q}^{2}(k)\right] \cdot E\left[\hat{X} C(k) \cdot \hat{X}^{T}(k) \cdot A \cdot \hat{X}(k) \cdot \hat{X} C^{T}(k)\right]
$$

can be computed as:(K $\mathbf{K}^{\text {由 }}$ term)

$$
\begin{aligned}
& \sum_{p} \sum_{q} E\left[x_{k} \cdot l c_{p} \cdot a_{p q} \cdot x_{q} \cdot l_{l}\right]=\sum_{p} \sum_{q} E\left[l_{k} \cdot x c_{p}\right] \cdot a_{p q} \cdot E\left[x_{q} \cdot l c_{l}\right]+ \\
& \sum_{p} \sum_{q} E\left[\hat{f}_{k} l c_{l}\right] \cdot a_{p q} \cdot E\left[\left\{c_{p} \cdot q_{q}\right]\right.
\end{aligned}
$$

where $a_{p q}$ is the $\mathrm{pq}^{\text {th }}$ element of matrix $A$ : and $x_{k}$ is the kth element of vector $X$. since

$$
E\left[\hat{X} C \cdot \hat{X}^{T}\right]=\Lambda
$$

So non-diagonal elements will be zero or $E\left[\hat{\boldsymbol{X}}_{p} \cdot \hat{\boldsymbol{X}} \boldsymbol{C}_{\boldsymbol{g}}\right]=0$ for $p \neq q$ and the equation can be simplified to:

$$
E\left[\hat{x}_{k} \cdot \hat{x} c_{k}\right] \cdot a_{k l} \cdot E\left[\hat{x}_{l} \cdot x c_{l}\right]+s_{k l} \cdot E\left[\hat{x}_{k} \cdot \hat{x}_{l}\right] \cdot \sum_{p} a_{p p} \cdot E\left[\hat{x}_{p} \cdot x_{p}\right]
$$

where $s_{k l}=1$ if $\mathrm{k}=1$ and 0 if $k \neq l$. Above equation in matrix form will be:

$$
E\left[\hat{X C}(k) \cdot \hat{x}^{T}(k) \cdot \Lambda \cdot \hat{X C}(k) \cdot \hat{\chi}^{T}(k)\right]=\Lambda \cdot \Lambda \cdot \Lambda+\Lambda \cdot 1^{T} \cdot G(k)
$$


By multiplying both side of eq(A26) by $\Lambda$ we derive the following equation for $G(k)$ :

$$
\begin{aligned}
G(k+1)= & {\left[I-E\left[\beta_{e q}(k)\right] \cdot \Lambda+E\left[\beta_{e q}^{2}(k)\right] \cdot \Lambda^{2}\right] \cdot G(k) } \\
& +E\left[\beta_{e q}^{2}(k)\right] \Lambda^{2} \times 1 \times M S E(k)
\end{aligned}
$$

to derive the steady state value of error:

$$
\lim _{k \rightarrow \infty} \operatorname{MSE}(k)=\lim _{k \rightarrow \infty}\left(e r r_{\min }+1^{T} \times G(k)\right)
$$

We use the Z-transform of above equation and final value theorem:

where

$$
\operatorname{MSE}(z)=\frac{\text { err }_{\text {min }}}{1-z^{-1}}+\sum_{i=1}^{n} G_{i}(z)
$$

$$
\begin{gathered}
G_{i}(z)=z^{-1} \cdot\left[1-E\left[\beta_{e q}(k)\right] \cdot \lambda_{i}+E\left[\beta_{e q}^{2}(k)\right] \cdot \lambda_{i}^{2}\right] \times G_{i}(z)+ \\
E\left[\beta_{e q}^{2}(k)\right] \cdot \lambda_{i}^{2} \cdot z^{-1} \cdot \operatorname{MSE}(z)+G_{i}(0)
\end{gathered}
$$

or

$$
\begin{gathered}
G_{i}(z)=\frac{E\left[\beta_{e q}^{2}(k)\right] \cdot \lambda_{i}^{2} \cdot z^{-1} \cdot M S E(z)+G_{i}(0)}{1-z^{-1} \cdot\left(1-E\left[\beta_{e q}(k)\right] \cdot \lambda_{i}+E\left[\beta_{e q}^{2}(k)\right] \cdot \lambda_{i}^{2}\right)} \\
M S E(z)=\sum_{i=1}^{n} \frac{E\left[\beta_{e q}^{2}(k)\right] \cdot \lambda_{i}^{2} \cdot z^{-1} \cdot M S E(z)}{1-z^{-1}\left(1-E\left[\beta_{e q}^{2}(k)\right] \cdot \lambda_{i}+E\left[\beta_{e q}^{2}(k)\right] \cdot \lambda_{i}^{2}\right)}+ \\
\sum_{i=1}^{n} \frac{G_{i}(0)}{1-z^{-1} \cdot\left(1-E\left[\beta_{e q}^{2}(k)\right] \cdot \lambda_{i}+E\left[\beta_{e q}^{2}(k)\right] \cdot \lambda_{i}^{2}\right)}+\frac{e r r_{\text {min }}}{1-z^{-1}}
\end{gathered}
$$




$$
\begin{gathered}
\left(1-\sum_{i=1}^{n} \frac{E\left[\beta_{e q}^{2}(k)\right] \lambda_{i}^{2} \cdot z^{-1}}{\left.1-z^{-1} \cdot i 1-E\left[\beta_{e q}(k)\right] \times \lambda_{i}+E\left[\beta_{e q}^{2}(k)\right] \times \lambda_{i}^{2}\right)}\right) M S E(z)= \\
\frac{e r r_{\min }}{1-z^{-1}}+\sum_{i=1}^{n} \frac{G_{i}(0)}{1-z^{-1}\left(1-E\left[\beta_{e q}(k)\right] \cdot \lambda_{i}+E\left[\beta_{e q}^{2}(k)\right] \cdot \lambda_{i}^{2}\right)}
\end{gathered}
$$

assuming:

$$
\begin{aligned}
& \lim _{k \rightarrow-\infty} E\left[\beta_{e q}(k)\right]=\bar{\beta} \\
& \lim _{k \rightarrow \infty} E\left[\beta_{e q}^{2}(k)\right]=\mu
\end{aligned}
$$

and using final value theorem:

$$
\begin{aligned}
& \operatorname{MSE}(\infty)=\lim _{z \rightarrow 1}\left(1-z^{-1}\right) \times \operatorname{MSE}(z)= \\
& \frac{e r r_{\min }}{1-\sum_{i=1}^{n} \frac{\mu \cdot \lambda_{i}^{2}}{1-\left(1-\bar{\beta} \cdot \lambda_{i}+\mu \cdot \lambda_{i}^{2}\right)}} \\
& \operatorname{MSE}(\infty)=\frac{e r_{\min }}{1-\sum_{i=1}^{n} \frac{\mu \cdot \lambda_{i}}{-\mu \cdot \lambda_{i}+\bar{\beta}}}
\end{aligned}
$$


misadjustment factor will be:

$$
M=\frac{M S E(\infty)-e r r_{\min }}{e r r_{\min }}
$$

By rewritting $\operatorname{MSE}(\infty)$ as:

$$
M S E(\infty)=e r r_{\min }\left[1+\frac{\sum_{i=1}^{n} \frac{\mu \cdot \lambda_{i}}{-\mu \cdot \lambda_{i}+\bar{\beta}}}{1-\sum_{i=1}^{n} \frac{\mu \cdot \lambda_{i}}{-\mu \cdot \lambda_{i}+\bar{\beta}}}\right]
$$

$M$ can be computed according to the following equation:

$$
M=\frac{\sum_{i=1}^{n} \frac{\mu \cdot \lambda_{i}}{-\mu \cdot \lambda_{i}+\bar{\beta}}}{1-\sum_{i=1}^{n} \frac{\mu \cdot \lambda_{i}}{-\mu \cdot \lambda_{i}+\bar{\beta}}}
$$

$\mu$ and $\bar{\beta}$ is computed in terms of cluster variance and adaptation parameters ( $\beta$ ) as follow:

$$
\begin{aligned}
\beta_{e q}(k)= & \left(\beta /\left(2 \sigma^{2}\right)\right) \cdot \exp \left(\left|y(k)-y_{d}(k)\right|^{2} /\left(2 \sigma^{2}\right)\right)= \\
& {\left[\beta /\left(2 \sigma^{2}\right)\right] \times\left[1+\frac{\left|y(k)-y_{d}(k)\right|^{2}}{2 \sigma^{2}}\right] } \\
& \lim _{k \rightarrow \infty}\left(E\left[\left|y(k)-y_{d}(k)\right|^{2}\right]\right)=e r r_{\text {min }}+e r r_{e x}
\end{aligned}
$$

where excess error is defined as:

$$
\text { err }_{\text {ex }}=M S E(\infty)-e r r_{\text {min }}
$$

so: 


$$
\begin{gathered}
\bar{\beta}=\left[\beta /\left(2 \sigma^{2}\right)\right] \cdot\left[1+\left(1 /\left(2 \sigma^{2}\right)\right) \cdot\left(\operatorname{err}_{\text {min }}+\operatorname{err}_{e x}\right)\right] \\
\mu=\left[\beta /\left(2 \sigma^{2}\right)\right]^{2} \cdot E\left[\left(1+\frac{\left|y(k)-y_{d}(k)\right|^{2}}{2 \sigma^{2}}\right)^{2}\right] \\
k \rightarrow \infty
\end{gathered}
$$

During the simulation it was observed that

$$
\mu=\alpha \cdot(\bar{\beta})^{2}
$$

and the best value for $\alpha$ found to be 1.1 .

$$
e r r_{\text {min }}+e r r_{\text {ex }}=\frac{e r r_{\text {min }}}{1-\eta}
$$

where

by assuming :

$$
\eta=\sum_{i=1}^{n} \frac{\mu \cdot \lambda_{i}}{\bar{\beta}-\mu \cdot \lambda_{i}}
$$

$$
\begin{array}{r}
\bar{\beta} * \mu \cdot \lambda_{i} \\
\eta=\sum_{i=1}^{n} \frac{\mu \cdot \lambda_{i}}{\bar{\beta}}
\end{array}
$$

So

$$
\begin{gathered}
\bar{\beta} \cdot \eta=\sum_{i=1}^{n} \mu \cdot \lambda_{i} \\
\bar{\beta} \cdot \eta=\mu \cdot \sum_{i=1}^{n} \lambda_{i}=\mu \cdot \operatorname{tr}(R)
\end{gathered}
$$


by replacing $\bar{B}$ and $\mu$ from eq(A50) and $e q(A 51)$ in $e q(A 55)$ we will have:

$$
\left(\beta /\left(2 \sigma^{2}\right)\right) \cdot \eta=\left(\beta^{2} /\left(4 \sigma^{4}\right)\right) \cdot\left[1+\frac{\left(e r r_{\text {min }}+e r r_{e x}\right)}{2 \times \sigma^{2}}\right] \cdot \operatorname{tr}(R) \times 1.1
$$

and by replacing $e r_{\min }+e r_{e x}$ from $e q(A 52)$ in $e q(A 56)$ we derive the following equation:

$$
\begin{gathered}
\left(\beta /\left(2 \sigma^{2}\right)\right) \times \eta=\left(\beta^{2} /\left(4 \sigma^{4}\right)\right) \times \operatorname{tr}(R) \times 1.1 \times\left[1+\frac{e^{e r r_{\text {min }}}}{2 \sigma^{2} \cdot(1-\eta)}\right] \\
\eta=\left(\beta /\left(2 \sigma^{2}\right)\right) \cdot\left[1+\frac{\text { err }_{\min }}{2 \sigma^{2} \cdot(1-\eta)}\right] \times \operatorname{tr}(R) \times 1.1
\end{gathered}
$$

From this equation for a given set of parameters $\left(B, \sigma, e r r_{\min }\right)$ we can compute $\eta$ and misadjustment factor. 


\section{APPENDIX B:}

Convergence condition for R.B.F equalizer in time variable channel

In this appendix, convergence condition for radial basis function equalizer in time variable channel is derived.

The model used for channel is a markovian model . In this model at each time step channel's taps are updated based on a first order markov chain $W_{k+1}=\alpha^{*} W_{k}+\operatorname{sqn}\left(1-\alpha^{\wedge} 2\right)^{*} Z_{k}$ where ' $\alpha$ ' is a constant close to 1 and $\mathrm{Z}_{k}$ is an independent zero mean Gaussian variable which forms the innovation part of markovian process. The effect of having a variable channel is considered in our analysis by having a variable optimum weight for the filter (weiner solution) which changes as $W_{\text {opt }}(k)=\alpha^{*} W_{\text {opr }}(k-1)+\operatorname{squ}\left(1-\alpha^{\wedge} 2\right)^{*} Z_{k}$

Considering these assumptions, updating equation becomes:

$$
W(k+1)=W(K)+\beta_{e q}(k) \cdot x^{*}(k) \cdot \operatorname{err}(k)
$$

and

$$
\begin{gathered}
E[W(k+1)]=E[W(k)]+E\left[\beta_{e q}(k)\right] \cdot E\left[X^{*}(k) \cdot \operatorname{err}(k)\right] \\
E[W(k+1)]=E[W(k)]-E\left[\beta_{e q}(k)\right] \cdot R \cdot E\left[W(k)-W_{o p t}(k)\right]
\end{gathered}
$$

( $R$ is auto-correlation matrix),considering that

$$
E\left[w_{o p t}(k+1)\right]=\alpha \cdot E\left[w_{o p t}(k)\right]
$$

the error weight vector $W^{e}(k)=W(k)-W_{\text {opt }}(k)$ satisfies the equation

$$
E\left[W^{e}(k+1)\right]=\left[I-E\left[\beta_{e q}(k)\right] R\right] E\left[W^{e}(k)\right]+(1-\alpha) E\left[w_{o p t}(k)\right]
$$


equation (BS) is stable if and only if

$$
\prod_{k=0}^{n}\left[I-E\left[\beta_{e q}(k)\right] R\right] \rightarrow 0 \quad n \rightarrow \infty
$$

A sufficient condition for equation (B5) to hold is

$$
E\left[\beta_{e q}(k)\right]<\frac{2}{\lambda_{\max }}
$$

where $\lambda_{\max }$ is the maximum eignvalue of the covariance matrix(R). 


\section{APPENDIX C:}

Steady state behavior analysis in time variable channel:

The model used for channel is a markovian model and data auto-correlation matrix is assumed to be diagonal.

Updating equation and equation for error weight vector are:

$$
\begin{gathered}
W(k+1)=W(K)+\beta_{e q}(k) \cdot X^{*}(k) \cdot \operatorname{err}(k) \\
W^{e}(k)=W(k)-W_{o p t}(k) \\
W^{e}(k+1)=\left[I-\beta_{e q}(k) \cdot X^{*}(k) \cdot x^{T}(k)\right] W^{e}(k)+ \\
(1-a) w_{o p t}(k)-\sqrt{1-a^{2}} \cdot z_{k}+\beta_{e q}(k) \cdot X^{*}(k) \cdot e(k)
\end{gathered}
$$

Similar to time inavariant analysis first we examine the orthogonality of error and input vector: computing

$$
\begin{gathered}
E\left[e(k) \cdot X^{* T}(k)\right] \\
E\left[e(k) \cdot X^{* T}(k)\right]=E\left[X^{* T}(k) \cdot Y_{d}(k)\right]- \\
E\left[X^{* T}(k) \cdot X(k)\right] \cdot W_{o p t}^{T}(k)
\end{gathered}
$$

by defining cross corelation vector as

$$
E[P(k)]=E\left[X^{* T}(k) \cdot Y_{d}(k)\right]
$$

optimum weight vector can be written as:

$$
W_{O p t}(k)=R^{-1} \cdot P(k)
$$


so the equation $\mathrm{C} 4$ will be simplified to:

$$
E[P(k)]-\Lambda \cdot \Lambda^{-1} \cdot E[P(k)]=0
$$

This means orthogonality of $q_{\mathrm{k}}$ and Input vector is valid at each time instant.

Computing MSE:

$$
\begin{gathered}
W(k+1)=W(k)+\beta_{e q}(k) \cdot x^{*}(k) \cdot e r r(k) \\
M S E(k))=E\left[(e(k)+e r r(k)-e(k))^{2}\right] \\
M S E(k)=\underset{\min }{E\left[(\operatorname{err}(k)-e(k))^{2}\right]} \\
M S E(k)=\operatorname{err}_{\min }-2 E\left[e(k) \cdot X^{T}(k) \cdot W^{e}(k)\right]+ \\
E\left[\left(W^{*}(k)\right)^{T} \cdot X^{*}(k) \cdot x^{T}(k) W^{e}(k)\right] \\
M S E(k)=\operatorname{err}_{\min }+1^{T} \cdot G(k)
\end{gathered}
$$

$G(k)$ is the same as appendix $(A)$ i.e: $G(k)$ is diagonal terms of matrix

$$
\Lambda \cdot E\left[W^{*}(k) \cdot\left(W^{*}(k)\right)^{T}\right]
$$

by assuming $1-a=0$ and using independency of input vector and orthogonality of $e(k)$ and Input vector the expected value of above equation can be computed as: 


$$
\begin{gathered}
w^{*}(k+1) \cdot\left(w^{*}(k+1)\right)^{T}= \\
\left\{\left[I-\beta_{e q}(k) \cdot X^{*}(k) \cdot X^{T}(k)\right] w^{*}(k)+(1-a) w_{o p t}(k)\right. \\
\left.-\sqrt{1-a^{2}} \cdot Z(k)+\beta_{e q}(k) \cdot X^{*}(k) \cdot e(k)\right\} \\
\left\{\left(W^{*}(k)\right)^{T}\left[I-\beta_{e q}(k) \cdot X^{* T}(k) \cdot X(k)\right]\right. \\
+(1-a) \cdot W_{o p t}^{T}(k)-\sqrt{1-a^{2}} \cdot z^{T}(k)+ \\
\left.\beta_{e q}(k) X^{T}(k) e(k)\right\}
\end{gathered}
$$

$$
\begin{gathered}
E\left[W^{e}(k+1) \cdot\left(W^{*}(k+1)\right)^{T}\right]= \\
E\left[W^{e}(k) \cdot\left(W^{*}(k)\right)^{T}\right]-E\left[\beta_{e q}(k)\right] \cdot \Lambda \cdot E\left[W^{e}(k) \cdot\left(W^{*}(k)\right)^{T}\right]- \\
E\left[\beta_{e q}(k)\right] \cdot E\left[W^{e}(k) \cdot\left(W^{*}(k)\right)^{T}\right] \Lambda \\
+E\left[\beta_{e q}^{2}(k) \cdot X^{*}(k) \cdot x^{T}(k) \cdot W^{*}(k) \cdot\left(W^{*}(k)\right)^{T} \cdot x^{*}(k) \cdot x^{T}(k)\right] \\
\sigma_{2}^{2} I+E\left[\beta_{e q}^{2}(k) \cdot x^{*}(k) \cdot x^{T}(k) \cdot e^{2}(k)\right]
\end{gathered}
$$

So the only difference with the time invariant case is the term

$$
\sigma_{z}^{2} I=\left(1-a^{2}\right) E\left[Z \cdot Z^{T}\right]
$$

By using gaussian moment theory and assuming 


$$
\begin{gathered}
E\left[\beta_{e q}(k)\right]=\bar{\beta} \\
k \rightarrow \infty \\
E\left[\beta_{e q}^{2}(k)\right]=\mu \\
k \rightarrow \infty
\end{gathered}
$$

We have :

$$
\begin{gathered}
E\left[W^{e}(k+1) \cdot\left(W^{e}(k+1)\right)^{T}\right]= \\
E\left[W^{e}(k) \cdot\left(W^{e}(k)\right)^{T}\right]-E\left[\beta_{e q}(k)\right] \cdot \Lambda \cdot E\left[W^{e}(k) \cdot\left(W^{e}(k)\right)^{T}\right] \\
-E\left[\beta_{e q}(k)\right] \cdot E\left[W^{e}(k) \cdot\left(W^{e}(k)\right)^{T}\right] \cdot \Lambda \\
+E\left[\beta_{e q}^{2}(k)\right] \cdot E\left[X^{*}(k) X^{T}(k) W^{e}(k)\left(W^{e}(k)\right)^{T} X^{*}(k) X(k)^{T}\right] \\
+\sigma_{z}^{2} \times I+E\left[\beta_{e q}^{2}(k)\right] \cdot \Lambda \cdot e_{\text {err }}
\end{gathered}
$$

$$
\begin{gathered}
G(k+1)=\left[I-E\left[\beta_{e q}^{2}(k)\right] \Lambda+\beta_{e q}^{2}(k) \cdot \Lambda^{2}\right] G(k)+ \\
E\left[\beta_{e q}^{2}(k)\right] \Lambda^{2} \cdot e r r_{\text {min }} \\
+\sigma_{z}^{2} I \cdot \Lambda \times 1+E\left[\beta_{e q}^{2}(k)\right] \Lambda^{2} \cdot 1 \times M S E(k)
\end{gathered}
$$

to analyze steady state behavior we compute Z-transform of $\mathrm{Eq}(\mathrm{C20})$ and (C13) and since we are concem only about steady state behwior we replace $E\left[\beta_{e q}(k)\right]$ and $E\left[\beta_{e q}^{2}(k)\right]$ by $\bar{\beta}$ and $\mu$ and we will have fhe ollowing set of equations: 


$$
\begin{aligned}
\operatorname{MSE}(z)= & {\left[\operatorname{err}_{\min } /\left(1-z^{-1}\right)\right]+\sum_{i=1}^{n} G_{i}(z) } \\
G_{i}(z)= & z^{-1}\left[1-\bar{\beta} \cdot \lambda_{i}+\mu \cdot \lambda_{i}^{2}\right] G_{i}(z)+ \\
& \mu \lambda_{i}^{2} z^{-1} M S E(z) \\
+ & \sigma_{z}^{2} \lambda_{i} z^{-1} /\left(1-z^{-1}\right)+G_{i}(0)
\end{aligned}
$$

from final value theorem we have:

$$
M S E(\infty)=\frac{\left(e r r_{\text {min }}\right)}{1-\sum_{i=1}^{n} \frac{\mu \cdot \lambda_{i}^{2}}{\bar{\beta} \cdot \lambda_{i}-\mu \cdot \lambda_{i}^{2}}}+\frac{\sum_{i=1}^{n} \frac{\sigma_{2}^{2} \times \lambda_{i}}{\bar{\beta} \cdot \lambda_{i}-\mu \cdot \lambda_{i}^{2}}}{1-\sum_{i=1}^{n} \frac{\mu \times \lambda_{i}^{2}}{\bar{\beta} \cdot \lambda_{i}-\mu \cdot \lambda_{i}^{2}}}
$$

$$
M S E(\infty)=\frac{e_{\min }}{1-\sum_{i=1}^{n} \frac{\mu \cdot \lambda_{i}}{\bar{\beta}-\mu \cdot \lambda_{i}}}+\frac{\sum_{i=1}^{n} \frac{\sigma_{z}^{2}}{\bar{\beta}-\mu \cdot \lambda_{i}}}{1-\sum_{i=1}^{n} \frac{\mu \cdot \lambda_{i}}{\bar{\beta}-\mu \cdot \lambda_{i}}}
$$



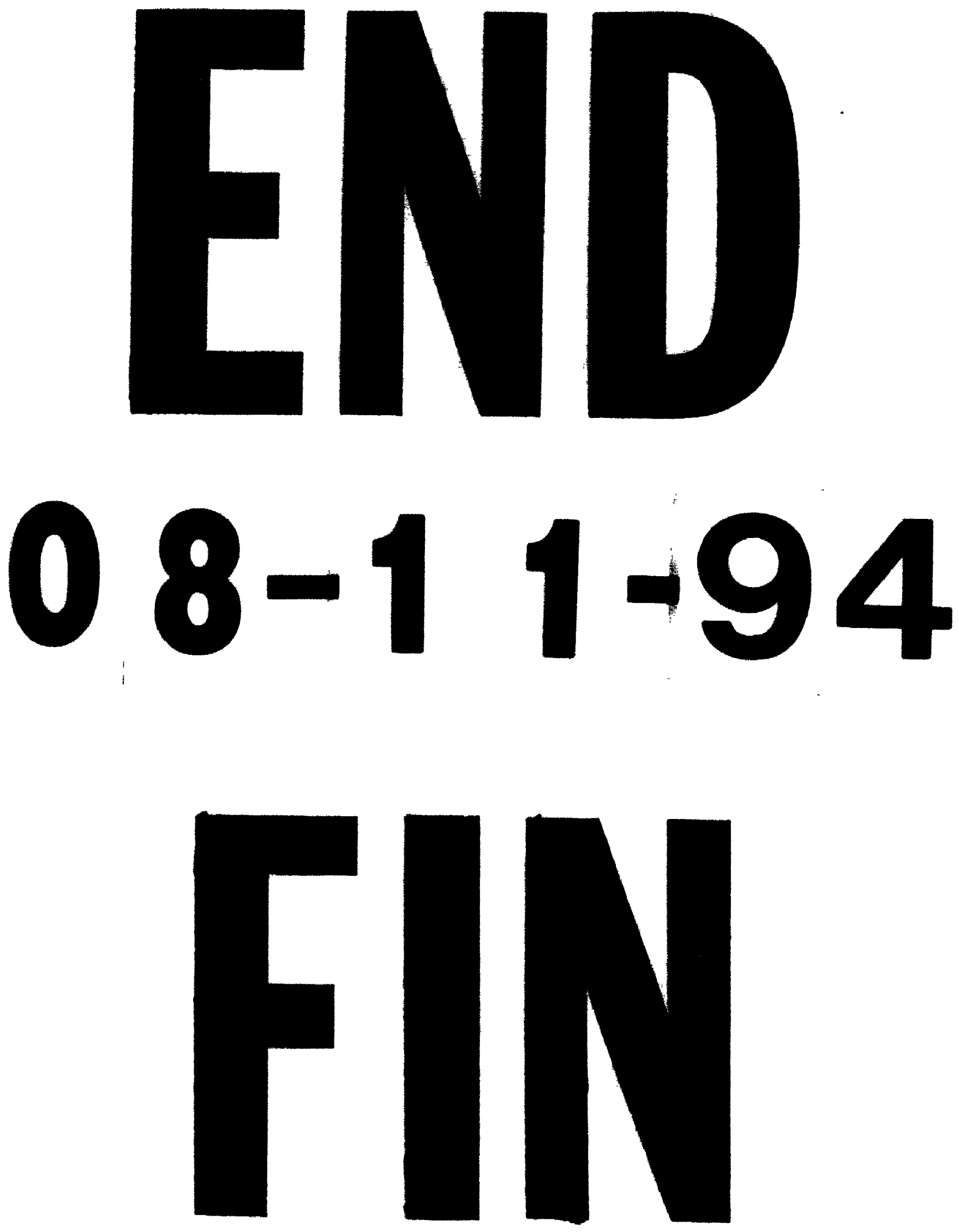